\title{
WestVirginiaUniversity
}

THE RESEARCH REPOSITORY @ WVU

Graduate Theses, Dissertations, and Problem Reports

2018

\section{Energy efficiency effectiveness of smart thermostat based BEMS}

Koushik Mandlem

Follow this and additional works at: https://researchrepository.wvu.edu/etd

\section{Recommended Citation}

Mandlem, Koushik, "Energy efficiency effectiveness of smart thermostat based BEMS" (2018). Graduate Theses, Dissertations, and Problem Reports. 4009.

https://researchrepository.wvu.edu/etd/4009

This Problem/Project Report is protected by copyright and/or related rights. It has been brought to you by the The Research Repository @WVU with permission from the rights-holder(s). You are free to use this Problem/Project Report in any way that is permitted by the copyright and related rights legislation that applies to your use. For other uses you must obtain permission from the rights-holder(s) directly, unless additional rights are indicated by a Creative Commons license in the record and/ or on the work itself. This Problem/Project Report has been accepted for inclusion in WVU Graduate Theses, Dissertations, and Problem Reports collection by an authorized administrator of The Research Repository @ WVU. For more information, please contact researchrepository@mail.wvu.edu. 
Energy Efficiency Effectiveness of Smart Thermostat Based BEMS

\author{
Koushik Mandlem
}

Problem Report Submitted

To the Benjamin M. Statler College of Engineering and Mineral Resources at West Virginia University

in partial fulfillment of the requirements for the degree of

\author{
Master of Science in \\ Industrial Engineering
}

\author{
Bhaskaran Gopalakrishnan, Ph.D., P.E., Chair \\ Ashish Nimbarte, Ph.D. \\ Kenneth H. Means, Ph.D., P.E.
}

Department of Industrial and Management Systems Engineering

Morgantown, West Virginia

2018

Keywords: HVAC, BEMS, Smart Thermostat, HVAC Zoning

Copyright 2018 Koushik Mandlem 


\section{Abstract \\ Energy Efficiency Effectiveness of Smart Thermostat Based BEMS}

Koushik Mandlem

Building Energy Management System (BEMS) is a computer-based system that can control, monitor and optimize energy consumption of the buildings. Though there are many advancements in this technology, many residential users who plan to install this technology are unaware of its economic feasibility. To determine the economic feasibility, the user should know the energy costs associated with the current HVAC system and the proposed HVAC system after installing BEMS and the implementation costs associated with the installation of the system.

The work that is being proposed here is to develop a software that allows the user to observe the estimated energy costs associated with a HVAC system after installing a smart thermostat based BEMS. The main factors considered to develop the model are occupancy of the rooms, weather factor, volume of the room, building envelope and thermostat set point. Also, the software will need some inputs from the user to run the simulation. The software will simulate the energy usage of the HVAC system for one year with a frequency of 30 minutes. This helps the users to evaluate the economic feasibility of the smart thermostat based BEMS, thus helping them to make a decision. A sensitivity analysis is performed to know the effect of each factor on the energy consumption. 


\section{Acknowledgment}

Firstly, I would like to express my sincere gratitude to Dr. Bhaskaran Gopalakrishnan, for his continued support and encouragement during the course of my research work. The door to Dr. Gopala's office was always open, whenever I ran into trouble or had any question regarding my research or my academics. My deepest and sincere "Thank you" to him.

I would like to thank Dr. Ashish Nimbarte for his interest in me at every stage of the research. His inspiration, timely suggestion with kindness, enthusiasm and energy have enabled me to complete my problem report.

Also, I wish to thank Dr. Kenneth Means for his advice and support.

Finally, I thank god, my family and friends for their constant support. 


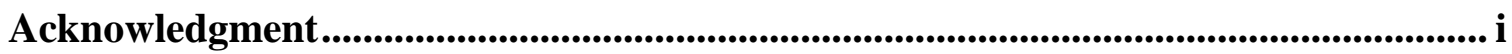

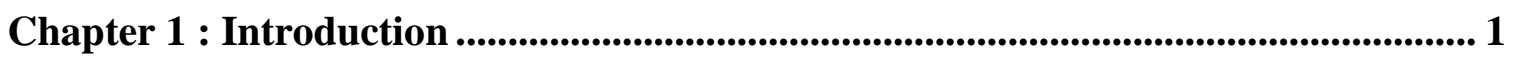

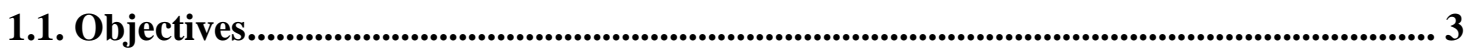

1.2. HVAC Systems

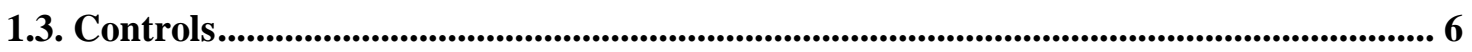

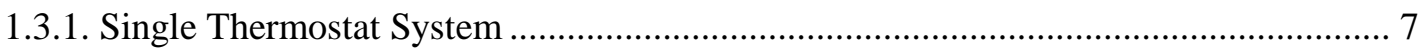

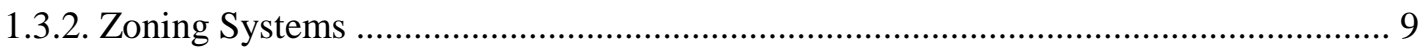

1.3.3. Building Energy Management System (BEMS) .................................................. 11

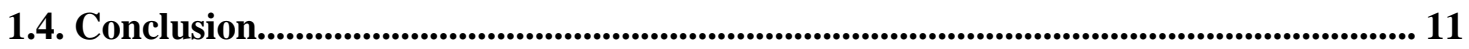

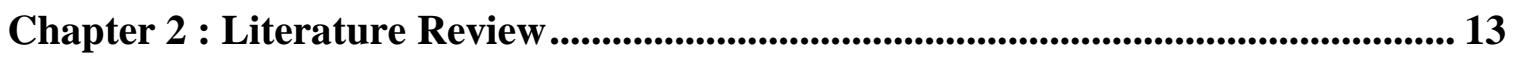

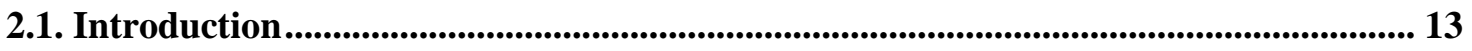

2.2. Factors Influencing HVAC Energy Consumption ............................................................. 14

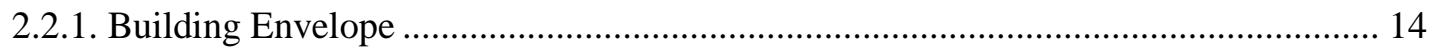

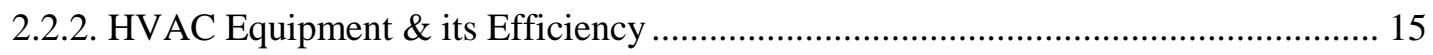

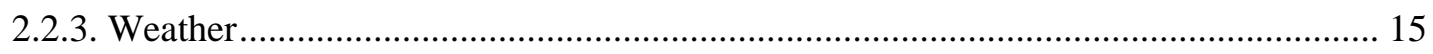

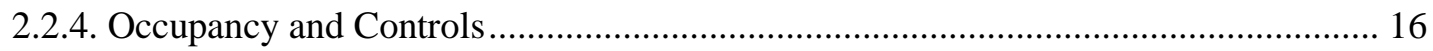

2.3. HVAC Energy Consumption Simulation Models ..................................................... 19

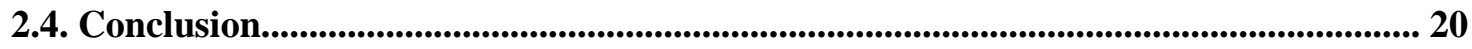

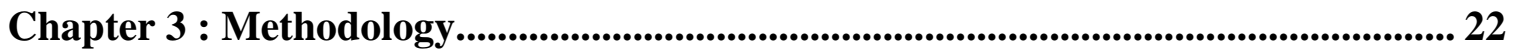

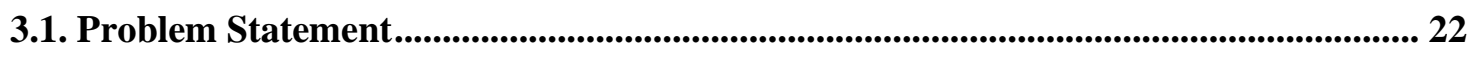

3.2. Description .............................................................................................................................. 23

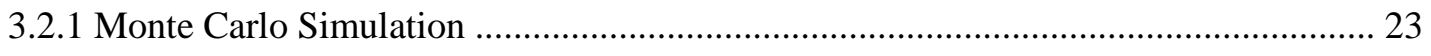

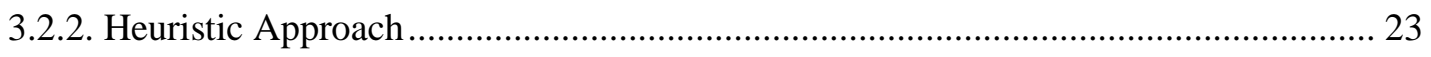


3.2.3. VBA

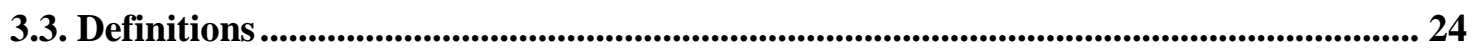

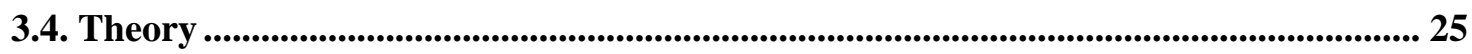

3.5. Software Model ................................................................................................................. 26

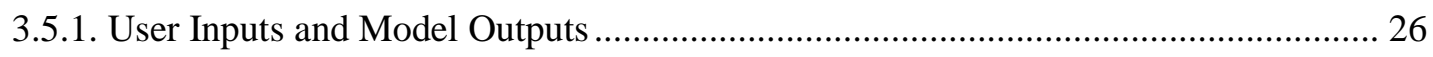

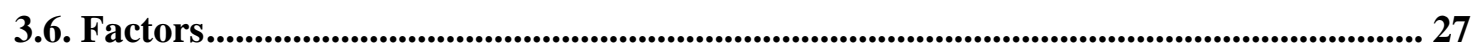

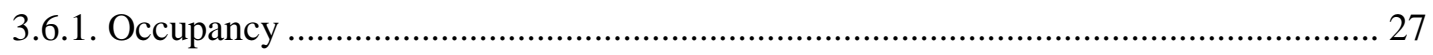

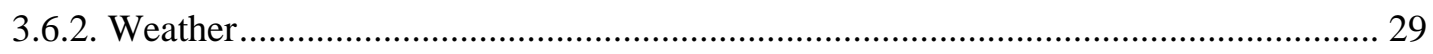

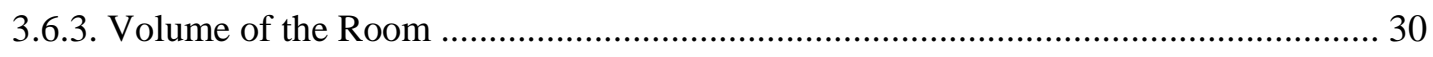

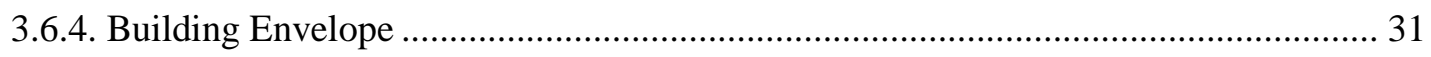

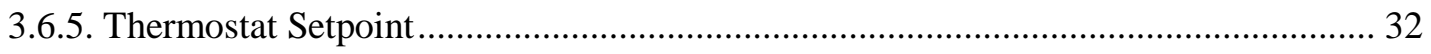

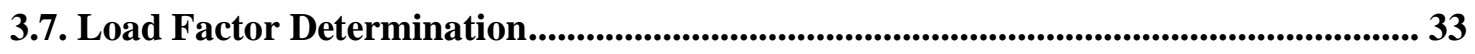

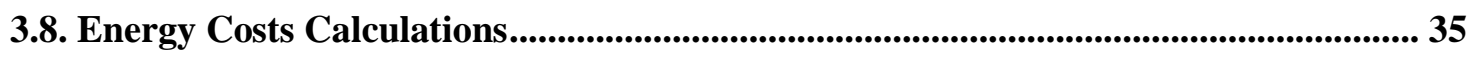

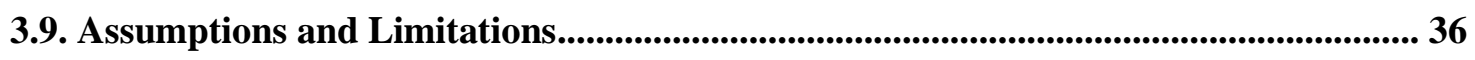

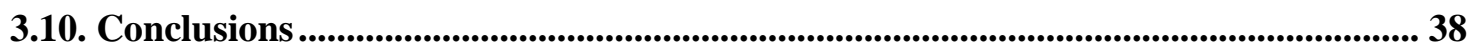

Chapter 4 : System Execution and Results....................................................................... 39

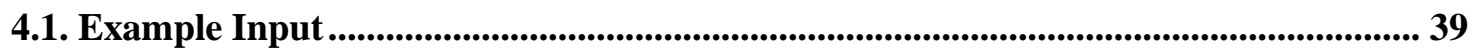

4.2. Sensitivity Analysis ........................................................................................................................... 47

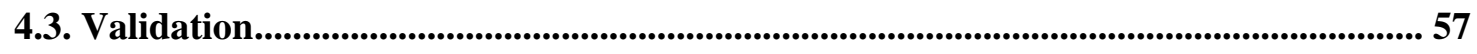

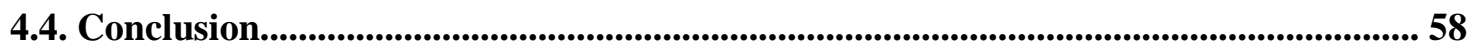

Chapter 5 : Conclusions and Future Work ................................................................ 59

5.1. Conclusions ..................................................................................................................................... 59

5.2. Future Work ......................................................................................................................... 59

Chapter 6 : References ................................................................................................................6 61 
Chapter 7 : Appendix ............................................................................................................. 66

7.1 VBA Code ........................................................................................................................................ 66

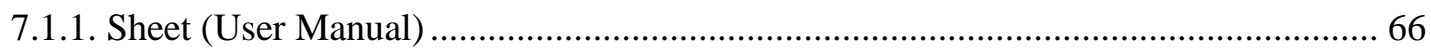

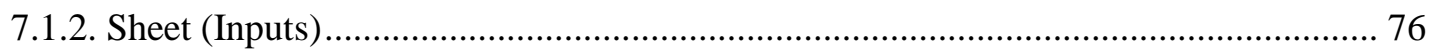

\section{List of Figures}

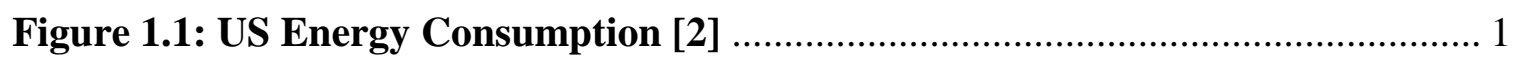

Figure 1.2: Air Conditioning Strategy in US homes [5] ..................................... 2

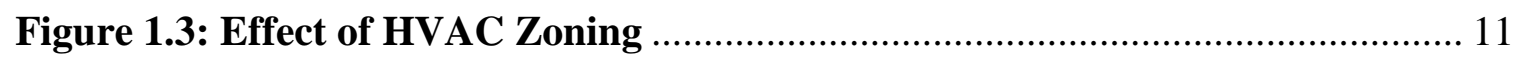

Figure 3.1: Rules for Occupancy - Sheet "References"............................................. 29

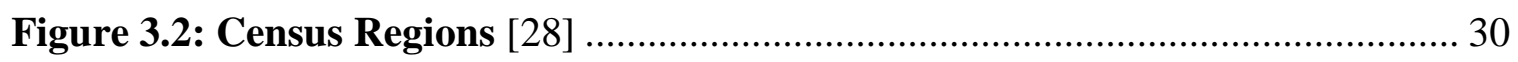

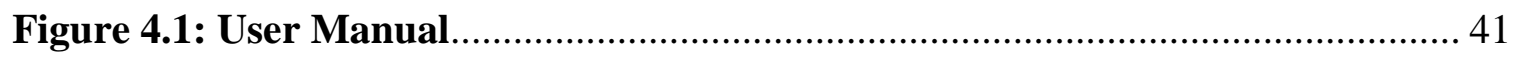

Figure 4.2: Input Boxes seeking Building Specifications ...................................... 42

Figure 4.3: Inputs from User for Factor .............................................................. 42

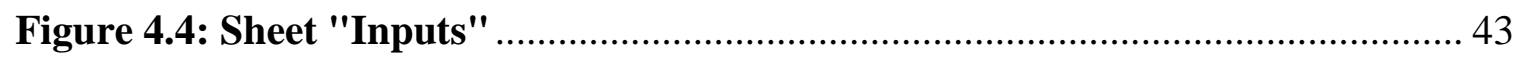

Figure 4.5: Sheet "Load Factor" ............................................................................ 45

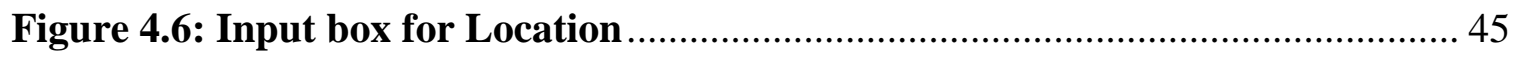

Figure 4.7: Input Boxes for Specifications of HVAC and Rate Structure................. 46

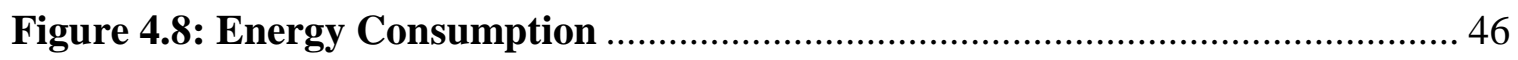

Figure 4.9: Electricity Consumption for Regular Controls vs Smart Controls ........ 49

Figure 4.10: NG Consumption for Regular Controls vs. Smart Controls ................. 49

Figure 4.11: Electric Consumption for different volume levels................................ 51

Figure 4.12: NG Consumption for different volume levels .................................... 51

Figure 4.13: Natural Gas Consumption for different Thermostat Comfort levels ... 52 
Figure 4.14: Electric Consumption for different Thermostat Comfort levels

Figure 4.15: Natural Gas Consumption vs. \# of times Thermostat set points changed

Figure 4.16: Electric Consumption vs. \# of times Thermostat set points changed ... 54

Figure 4.17: Electric Consumption vs Building Envelope Levels ........................... 56

Figure 4.18: NG Consumption vs. Building Envelope Levels .............................. 56

Figure 4.19: Total Energy Costs vs. Location of User …................................ 57

\section{List of Tables}

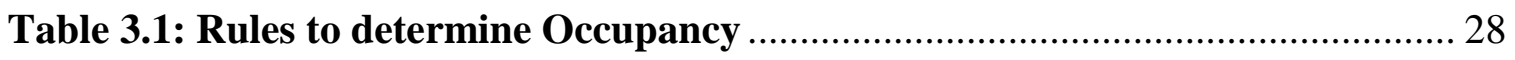

Table 3.2: Rules to determine Weather Factor ................................................. 29

Table 3.3: Standard Sizes of rooms in Residential Building[29] ........................... 31

Table 3.4: Rules to determine volume level ....................................................... 31

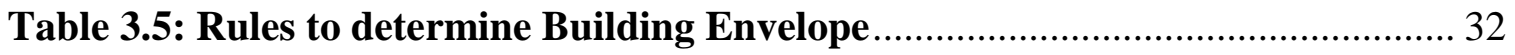

Table 3.6: User Guidelines to determine the level for the thermostat ..................... 32

Table 3.7: Model Guidelines to determine the level for the thermostat .................. 32

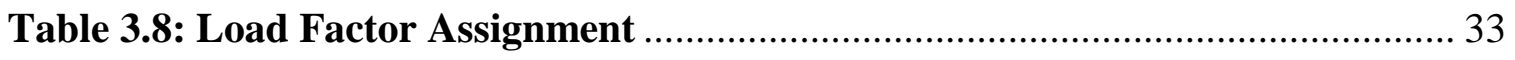

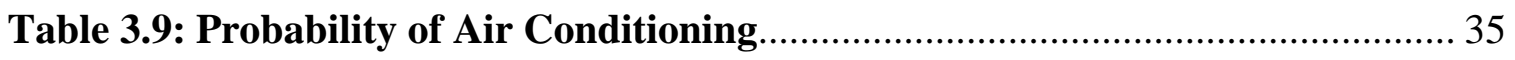

Table 4.1: Energy Consumption (Example Input) ........................................... 47

Table 4.2: Specifications of Buildings (3 Cases to Plot the Graphs) ........................ 50 


\section{Chapter 1 : Introduction}

Global energy consumption continues to increase each day. In the 1970s the global energy usage was about 3860 Million Tons of Oil Equivalent (MTOE), and within ten years the numbers almost doubled and took the global energy usage to 6630 MTOE [1]. By the end of 2012, the total energy consumed by the whole world was about 13371 MTOE [1].

There is not much variation in the US energy consumption in the last decade. The average US energy consumption in the previous decade was around 100 quadrillion BTUs; varying from 94 quadrillion BTUs to 101 quadrillions BTUs [2]. According to the U.S. Department of Energy, buildings account for about $40 \%$ of the total energy consumption in the United States [2]. The industrial and transportation sectors account for the remaining 60\%. Heating Ventilation and Air Conditioning (HVAC) systems represent 42.5\% (17\% of total energy consumption) of the energy consumption in buildings in the United States. Hence, enhancing the operation of the HVAC system can result in opportunities to save energy and therefore reduce the operating costs for a residential or industrial area.

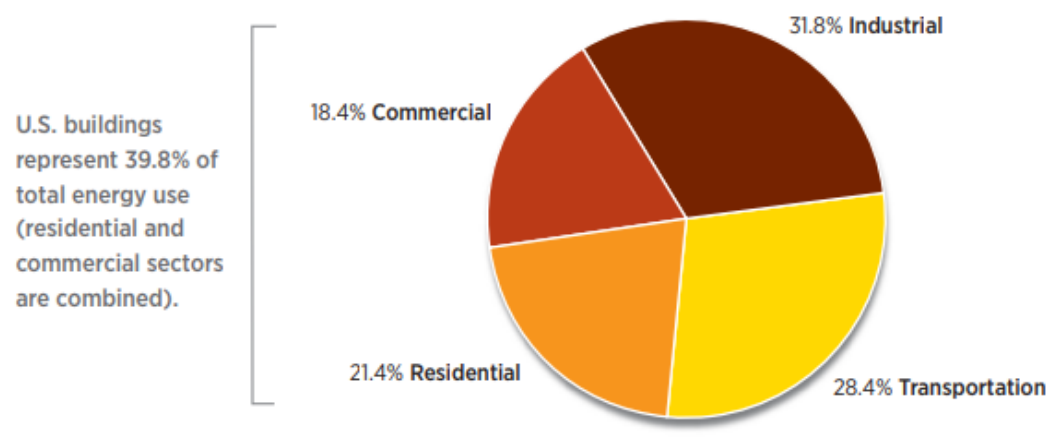

Figure 1.1: US Energy Consumption [2] 
People spend about most of the time indoors [3]. Therefore, thermal comfort is essential. It has also been proved that efficiency of the worker or the occupant increases if the work environment is at their desired state [4]. People install thermostats at a fixed place to sense the temperature of the room and regulate the HVAC system. Room temperature is not maintained at a fixed value. It is often changed to suit the occupant's requirement. Setting back the thermostat can put a break on energy consumption. To learn the human behavior on the set back, a survey was done by EIA. The respondents using central air conditioners and individual air conditioning units were asked: "How do you set indoor temperatures during summer?" $45 \%$ of the people using central air conditioners and $31 \%$ of the people using individual air conditioning units responded that they set the units at one temperature and leave it there for most of the time [5]. Automatically setting back the thermostats can help in solving this problem.

\section{Air-conditioning control strategies in U.S. homes}

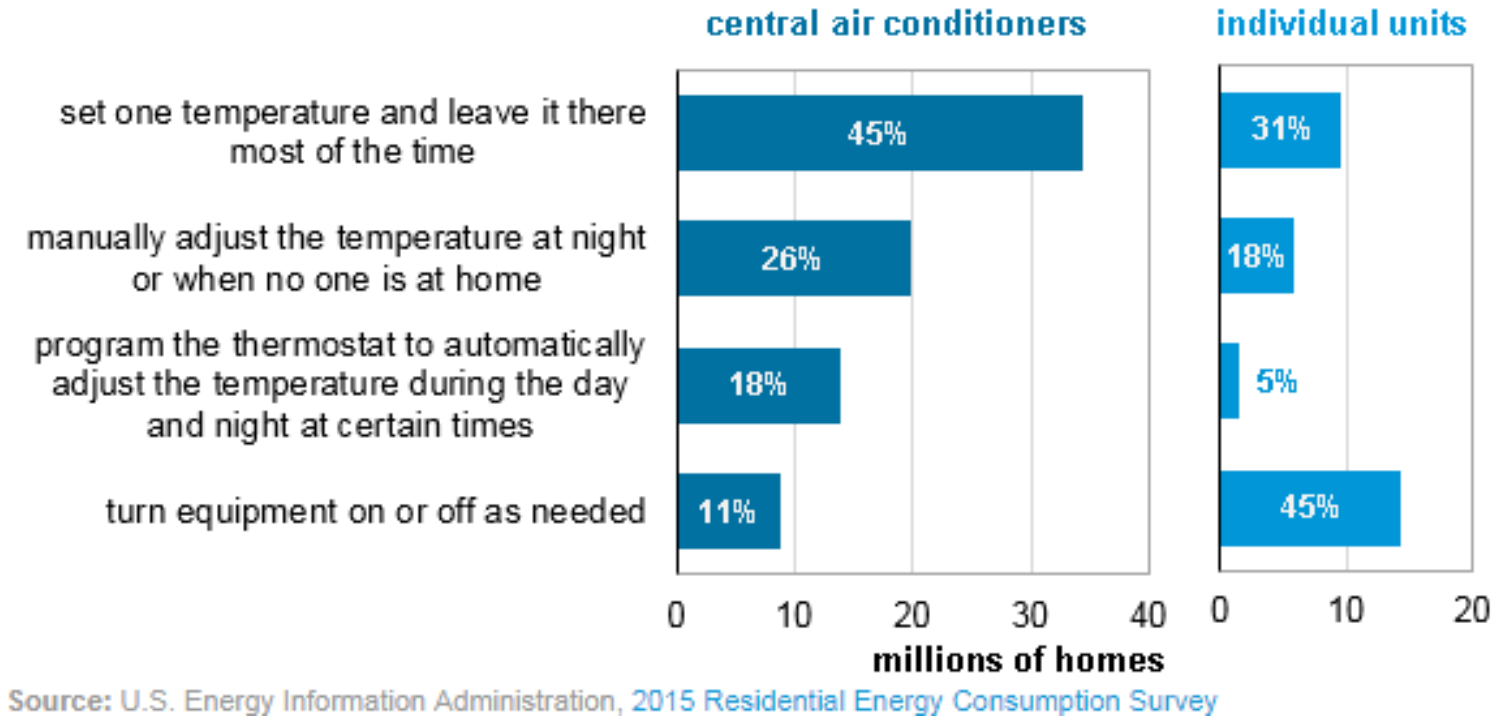

Figure 1.2: Air Conditioning Strategy in US homes [5] 
A typical residence uses a single thermostat in a fixed position. In this case, the temperature profile is not uniform across the thermal space. The insulation and infiltration in individual rooms may not be the same, so they respond differently to the conditioning systems. A single sensor control system may unnecessarily cool or heat the thermal environment and thus result in higher energy consumption. In addition to that, a single sensor system may not be able to learn about the human occupancy and behavior in different areas. Thus, it may not be able to regulate the HVAC systems to curtail the air flow in the unoccupied areas. To regulate the flow in the unoccupied spaces, the residence needs to be zoned. New generation control systems along with zoning could deploy several sensors or thermostats in different rooms. Then the system will learn the human behavior continuously and control the air flow into the rooms. Though implementing this strategy helps in enhancing the occupant's comfort with a reduction in energy consumption, it still has drawbacks. The drawbacks mainly include economic feasibility and the frequent equipment breakdowns. As these systems have several moving parts, the equipment has a high probability to break down. Thus, it is essential to know the estimated energy savings if the zoning systems with advanced controls are to be installed.

\subsection{Objectives}

The primary research objectives are:

- To develop a model to determine the HVAC energy consumption. The model will have inputs with varying parameters that are associated with building characteristics and HVAC characteristics. 
- The research aims to compare the impact of various factors on energy consumption.

The factors are

\section{Occupancy}

2. Weather

3. Volume of the Room

4. Building Envelope

5. Thermostat Setpoint

- The model will be used to perform sensitivity analysis.

\subsection{HVAC Systems}

Heating Ventilating and Air Conditioning (HVAC) systems provide human comfort by regulating heat and moisture. In buildings with high occupancy, people exhale carbon dioxide and release other contaminants such as smoke from cigarettes. In addition, there might be other odors produced due to the presence of machines. HVAC is responsible for the disposal of the carbon dioxide and other odors generated. If failed to do so, the health of the people residing or working in this environment may deteriorate. HVAC system achieves this by changing the air and removing odor, pollen and other contaminants regularly from the facility. HVAC system mainly includes

- Motors

- Dampers

- Fans

- Heat sources

- Heat exchanger units 


\section{- Controls}

The amount of heating and cooling required depends on the respective loads. Heat is generated predominantly by the sun, industrial processes, lights and people. Though this heat is beneficial during winter, it adds load on the air conditioning system during summer. HVAC systems must provide ventilation and airflow even if there aren't any cooling or heating loads.

HVAC systems vary on the type of fluid used as the medium of heat transfer. The fluid used depends on the functionality and the type of system installed when the building was originally built. In general, heating and air conditioning systems have some similar components like the source of heat, the heat exchange medium, and controls. The sources for heating usually are furnaces, boilers, and sun. Similarly, the sources of cooling are a chiller and an air conditioner.

Hot water, hot air and steam are generated using boilers or furnaces. Boilers and furnaces employ electricity or fossil fuels such as natural gas, propane, etc. to produce heat which is then exchanged with the air or water. Air or water can be directly heated by transferring the heat generated from the combustion of fossil fuels or electric resistance. Boilers can also add more heat to the water to produce steam. Similarly, chillers use a basic vapor compression cycle or an absorption cycle to provide cold water, cold air or cold fluids like glycol. The air, water, steam or other fluids can be distributed through the ductwork or piping to the areas where it is needed.

The controls may be as simple as adjusting the thermostat to turn the furnace on or off. They can also be complicated and have the settings for relative humidity, temperature and 
air changes which may be monitored by various sensors, actuators, valves, motors and dampers. As the study focuses more on controls, the controls are discussed detail in the following section.

\subsection{Controls}

HVAC systems are typically designed to meet the extreme conditions. They usually work at part load or are turned off depending on the heating and cooling loads, occupancy, weather and other factors. HVAC controls regulate the operation of the HVAC systems. Controls usually have a couple of sensors or a single sensor which senses the current state and compares it with the desired state. Finally, it turns the equipment on or off based on the desired state. There are several ways to control the temperature. One such method is to control the air volume supplied into the room and the other is to regulate the load factor of the equipment. Without proper controls, there might be overheating or overcooling of the space. In order to meet all the above functions, a basic control unit should include

- Sensor

- Controller

- Controlled device

- Source of power to operate the controlled device.

A sensor usually senses the state of the room and communicates with the controller. Once the controller receives the input, it processes the information and sends an electronic output to the controlled device. The controlled device now regulates the system as per the feedback received from the controller. There are two types of control systems

- Open loop control system 
In this system the output signal or state is not measured or fed back to the system to compare it with the setpoint. They usually have timers to regulate the system.

- Closed loop control system

In this system, the output signal or state is measured and fed back to the system to compare it with the setpoint. Then the controller sends the signal to the controlled device to regulate the system.

\subsubsection{Single Thermostat System}

The thermostat is a closed loop control system used to regulate the HVAC system based on temperature. It tries to minimize the error between the desired and measured state. A thermostat sometimes has all the elements of the control system in it. The position of the thermostat in the room is also important. It should be positioned such that it is not manipulated by any other external factors like sunlight or any other heat source. In a single thermostat control system, there is only one thermostat in the whole apartment governing the operation of the HVAC system. The thermostat senses the temperature of the area around it and regulates the HVAC system accordingly.

A research done by EPA (U.S. Environmental Protection Agency) found that about half of the households are vacant during the day. In winter about $42 \%$ of them turn the heat down and $2 \%$ of them turn their heating systems off [6]. This creates an opportunity to employ programmable thermostats. Programmable thermostats are the thermostats which have feasibility to be programmed. Once programmed the thermostats adjust the temperature based on the programs. The primary purpose of the programmable thermostats is to adjust the temperature automatically unlike a simple thermostat where one should set back the temperature manually. It has been thought that programmable thermostats should reduce 
up to $30 \%$ of the HVAC consumption by altering the heating and conditioning of the building as per the occupant's daily schedule. On the contrary, as per Energy Star's survey in 2009, the buildings with programmable thermostats consumed more energy than systems with regular manual thermostats [6]. The energy wastage was due to several factors. Some of them are:

- The temperatures are not set back if the occupants leave the house before the schedule.

- The set back temperatures are higher, which causes the system to run even when the house is vacant.

- If the occupants return back to the house early than the scheduled time they feel uncomfortable. This resulted in people reducing their set back schedule or even stopping the usage of the schedules. This explains as to why only $12 \%$ of the 118 million households program their thermostat though $41 \%$ of them own a programmable thermostat [5].

The updated version of the programmable thermostat is the smart thermostat. The only difference between a programmable and smart thermostat is the ability to connect to the internet and allowing the users to adjust the heating or cooling settings from other internet connected devices like smartphones and computers. Though the smart thermostats were introduced, the energy wastage could not be minimized completely. As a result, Smart Learning Thermostats were announced to the market. Smart Learning Thermostats are those which repeatedly learn the occupancy and the setback schedule of the occupant and act according to the learned behavior. This helps the thermostat to analyze the lifestyle of the occupant and then preheat or cool the building while being energy efficient. 
Though installing a single smart thermostat system to regulate the HVAC system in a residential area can reduce the energy consumption, it may not be optimal. Since a single thermostat cannot measure the temperature in all areas, the room where the thermostat is located is kept at the desired temperature. The rooms where the thermostat is not present are either hotter or colder with respect to the room where the thermostat is located. In such cases, the purpose of the HVAC system, i.e., providing comfort levels for the occupants is not met, and the system is either heating or cooling other areas more.

The other problem with the single thermostat system without zoning is that the rooms which do not need conditioning are also conditioned. However, many people close the vents in the rooms, not in use. The HVAC system and ductwork are designed considering the area of the entire building. If the vents are closed in the rooms that are not in use, the air does not have any chance to move out of the system, resulting in an increase of pressure in the ductwork and thereby developing air leaks in the ductwork. This may also result in overheating the furnace or freezing the air conditioner. Zone controls are an answer to these problems.

\subsubsection{Zoning Systems}

HVAC zoning system employs dampers in the ductwork to regulate and redirect the air flow into different areas (zones) in the building. Zoning helps in gaining more control on the HVAC system by turning the system off as soon as the desired temperature is met in the particular zone. However, zoning doesn't affect the efficiency of the HVAC system. Zoning also eliminates the problem of overheating or overcooling of the furnace or air conditioner. It reduces the energy consumption while addressing comfort requirements of 
the occupants by maintaining the zone at the desired state. The main components required for HVAC zoning include

- Central Control System: It is a control hub for all the components such as thermostats, HVAC system, dampers, etc. The control system is the only source of communication between the system components. It is the only processor for the entire zoning system.

- Thermostats: In order to regulate the temperatures, each room should be facilitated with a thermostat. The thermostat in each room sends the signal to the central control system. This helps the control system to control the dampers to vary the air flow into the room to maintain the desired temperature.

- Zone Dampers: Damper is a valve or a plate which can be regulated to vary the air flow. These are wired to the control system. They open or close based on the signal from the thermostat present in the room.

- Bypass Damper: In a single duct system, if the damper is closed for one of the zone, pressure builds up in the ductwork. Bypass damper acts as a relief valve which helps in venting out the air.

The effect of zoning a house can be observed in the figure below. 

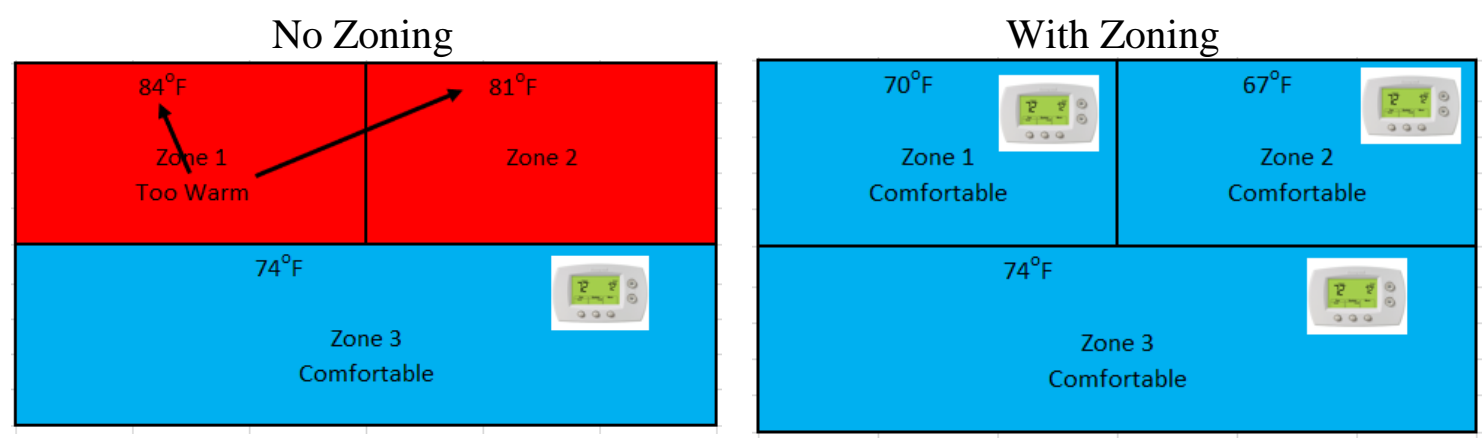

Figure 1.3: Effect of HVAC Zoning

\subsubsection{Building Energy Management System (BEMS)}

The main function of Building Energy Management System is to regulate, monitor and control HVAC systems. BEMS will employ computers to manage the energy consumption of the devices and building technical services. BEMS can integrate building's HVAC and lighting. However, some expensive state of the art BEMS can incorporate additional building systems such as video surveillance, access control and interfacing with fire and security controls.

This research considers a residential house which has been zoned. It is installed with a BEMS which takes feedback from the smart thermostats with occupancy detection capability present in each zone. Thus, regulates the HVAC system's load factor.

\subsection{Conclusion}

This chapter helps in understanding the energy consumption across United States, followed with a brief explanation of the need and limitations for smart controls. This chapter also explains the need for the research and also states the objectives of this study. HVAC system and its operation is briefly discussed in this chapter. This research will provide the user 
basic idea on the energy consumption of a HVAC system with smart thermostat based

BEMS. 


\section{Chapter 2 : Literature Review}

\subsection{Introduction}

A million residential buildings are being built in the United States on an average in a decade. This will have a significant impact on the carbon dioxide emissions and the amount of energy to be produced. The emissions and the energy requirement can be minimized by ensuring that the buildings are sustainable as well as efficient. This can be done by increasing the number of green buildings. In a study performed on sustainable performance of green buildings [12], A. Ghaffarianhoseini, et al. enlightened the essence of sustainability in green building design. Their work explains the trends and effects of green building design. The results showed that being green could, in fact, reduce carbon dioxide emissions as well as reduce the energy consumed. Buildings should be assessed on a regular basis to safeguard the building's efficiency. Records of the major energy consuming equipment must be maintained and evaluated on a regular basis. T. Markis and J.A. Paravantis have studied the energy consumption in small enterprises [8]. They evaluated six major energy consuming equipment which are building envelope, HVAC systems, lighting, space and water heating, electromechanical equipment, and distribution and transportation systems. The results showed that HVAC systems were more sensitized to energy conservation followed by electromechanical equipment and space and water heating equipment. 


\subsection{Factors Influencing HVAC Energy Consumption}

There are many options to reduce the energy consumption of the buildings sustainably. However because of the numbers, there is more research done on improving the efficiency of HVAC systems. The factors which influence the HVAC energy consumption are

- Building envelope

- HVAC equipment and its efficiency

- Level of the thermostat

- Outside temperature

- Occupancy

- Controls

\subsubsection{Building Envelope}

The physical barrier between the interior and exterior of a building is defined as building envelope. Building envelope plays the main role in improving the efficiency of HVAC systems. There are many studies done on building envelope design and retrofits. McKay [9], Al-Saadi S and Al-Jabri K [10] believes that thermal characteristics of the building and building envelope such as heat load in the building, indoor temperature, heat transmission and heat storage should be accounted in the design and construction phase of a building. The results of the study done by McKay [9] proved that the amount of insulation in the walls and roof would have a significant effect. He also explained that the load added to the air conditioner as heat failed in escaping or moving out of the building due to heavy insulation on walls and roof. Al-Saadi S and Al-Jabri K [16] developed several building envelope designs and have done a sensitivity analysis to narrow down several design 
factors like amount of insulation for the walls and doors, length of overhang on the windows, external shading, air infiltration etc. The results showed that minimum energy savings of $18 \%$ might be obtained by enhancing the design factors.

\subsubsection{HVAC Equipment \& its Efficiency}

Most often designers consider several safety factors to ensure proper functioning of the system at any point in time and any extreme climate. This is one of the leading causes of increased energy usage for HVAC systems. Conte R [11] studied effects of oversizing the equipment. It was also noted by him that if the boiler or furnace is oversized, it needs to run at part load, which might reduce the efficiency of the equipment, thereby increasing the usage. Air-conditioning system usually has a compressor and fans, which are responsible for the air conditioner's energy usage. Therefore, it is usually essential to minimize the utilization of the compressor. This can be achieved by sending outside air into the room or mixing the outside air and the return air if the outside temperature is lower than the inside temperature. An economizer has the ability to do this. There is significant research done on economizer's effect on air conditioning equipment. In a study done on an air-conditioning system performance with heat reclaim and economizer operation [12], V. S. Anantapantula developed hour by hour energy calculation programs for four types of HVAC systems used. The results showed that by reclaiming the heat could save up to $60 \%$ of the energy consumption. He also stated that combined operation of heat reclaim and economizer cycle would result in the least annual total HVAC energy used.

\subsubsection{Weather}

The other important factor as mentioned is the outside temperature (climate). In the study by James Jones, et al.[13], they performed tests on two buildings to check if the outside 
temperature, relative humidity, lighting power and solar radiation affects energy consumption. The results suggested that outdoor temperature and relative humidity are most significant for increasing electric demand and thus increasing the total energy usage.

\subsubsection{Occupancy and Controls}

Occupants must be able to control the temperature and set points to retain their comfort and conserve some energy as well. Control Systems will do this. Many studies and surveys proved that there is an increase in satisfaction level for the occupants when there is a thermostat in their room that they can control [4]. The areas however, need not be continued at that same temperature and can be brought back to energy friendly set points when there is no occupancy. A study was done by John Ingersoll and Joe Huang [14] in a residential building to manage heating energy by controlling the temperature. The analysis was done by varying the levels of insulation, the coating on windows and air infiltration in different climates. The results showed that the energy savings through setting back the temperature were substantial; are maximum for the houses where building envelope is loose, and are in hotter climates. Wei Guo and Darin W.Nutter [15] examined a double corridor classroom building for the set back and set up temperature. They observed that there are not as many savings when doing the set back always. It is dependent on the building structure and the outside temperature. They are not actively dependent on the building orientation and the window area. They also observed that there are certain set up and set back temperatures beyond which the energy savings remain constant. They also proposed the school to use the programmable thermostats they had, to set up and set back the temperatures. 
After realizing the importance of energy savings which can be achieved by installing programmable thermostats, Energy Star started promoting programmable thermostats as a means of reducing energy usage for residential customers anywhere from 10-30\% [16]. David Cross and David Judd [17] studied the occupant's behavior towards programmable thermostats. In this study, they surveyed 100 people who received programmable thermostats. Out of 100 , about $20 \%$ of them replaced the programmable thermostats with manual thermostats as the occupants felt uncomfortable because of the room temperature. This might be due to the occupant returning back to the house earlier than expected or the thermostat not being programmed. In addition, about $21 \%$ of them did not set back their thermostat and maintained a constant temperature, resulting in wastage of energy. After such similar studies were done by US EPA and Energy Star [6], realizing that there were no savings or sometimes increased usage from programmable thermostats, Energy Star decided to stop the program in 2009. After the decline of the usage of programmable thermostats, many new strategies that had potential to save energy were born. One such strategy is to predict the occupancy.

These thermostats which can sense or predict the occupancy, regulate the HVAC systems accordingly. However, there are many ways to detect the occupancy; some of them require customers to wear a tag [18] or using fingerprints or retina scanners or use of cameras, etc. Unfortunately, these techniques either require a lot of investment or create discomfort to the customers. The most economical way is to use reactive thermostats, which employs motion sensors, door sensors or key cards. Gao G and Whitehouse K [19] in their study created a smart thermostat which acquires information by X10 sensors deployed in several rooms in 8 houses they experimented on. The experiment helped in analyzing the 
occupancy patterns. They also used reactive thermostats to analyze the occupancy patterns. These patterns were then used as an input for the EnergyPlus simulator to estimate the HVAC consumption. The results showed that installing reactive thermostats increased the energy consumption up to $10 \%$. However, they were confident of their technology and stated "smart thermostat we designed has a potential to save up to $28 \%$ of the usage".

Gupta M, et al. [20] proposed an innovative way to estimate the occupancy be calculating the arrival time of the resident. The thermostat is connected to either the resident's phone or the GPS of the car. The thermostat then estimates the arrival time by getting the location of the resident and then adjusts the furnace or the air conditioner to reach the set point desired by the resident. This could save about $7 \%$ of the HVAC energy usage, which is less than the savings obtained from both programmable thermostat and reactive thermostat.

Qela B and Mouftah H [21] attempted to study on self-learning thermostats. They used an algorithm - Observes, Learns and Adapts (OLA). Integrating several wireless sensors and artificial intelligence concepts resulted in the generation of OLA algorithm. They used a house simulator as a base to estimate the energy savings obtained by not using and using the OLA algorithm. The usage of OLA algorithm could save additional $79 \mathrm{kWh}$ in a month.

A study was done in Indiana by Vectren South [22] to evaluate the percentage of savings obtained on heating and cooling in residential houses using Nest (smart learning) thermostats compared to manual and programmable thermostats. This study considered 300 households using Nest thermostats and programmable thermostats each, and about 3,845 households using manual thermostats. The study involved not only assessing the savings; they also considered the behavior of the participants, metered data, and the survey 
data. The results showed that by using Nest thermostats, the participants could save about $7 \%$ more on gas compared to the programmable thermostats and could save almost the same as a programmable thermostat on the electricity.

\subsection{HVAC Energy Consumption Simulation Models}

In general engineering and statistical methods are employed in research to estimate or simulate the Building energy consumption. Engineering methods use physical principals; like thermodynamics to study the thermal dynamics and energy behavior of a building and its subcomponents. US government supported Department of Energy for the past 20 years to develop two softwares DOE-2 and BLAST. However these two were merged to create EnergyPlus. EnergyPlus is a whole building energy simulation program which can estimate the water and energy usage of any building. It also has the capacity to estimate the thermal loads (cooling and heating) to maintain the set points of the HVAC system, coil loads and energy consumption of plant equipment. However, with the requirement of many inputs, environment details and the complexity involved, researchers proposed new models to estimate the energy consumption.

A model was developed by Muratori, et al. [23] based on the overall thermal resistance theory. The model performs a control volume analysis based on fundamentals of thermodynamics. Authors developed a relationship between air flow rate from the ductwork, input capacity of the furnace and temperature of the air from HVAC system. Once the user selects the air flow and input capacity, the temperature of the air from HVAC system is fixed. The model then estimates the energy required for the HVAC system to raise or drop the ambient temperature of the room. White and Reichmuth [24] developed a new model which uses monthly average outside temperature only to calculate total monthly 
energy consumption. They developed few equations to calculate the total energy consumption.

From the beginning, researchers adopted either multiple linear regression methods or artificial neural networks to estimate the energy consumption of HVAC system. Ruijin, et al. [25] developed a model for commercial building by using time series analysis method combined with regression model. The model uses the monthly energy consumption of past three years to estimate the energy consumption of the fourth year. T.Olofsson and S.Andersson [26] developed a model to estimate the energy demand based on the energy demand from two to five weeks. The authors used neural network models and also measured the daily heating demand along with outdoor and indoor temperatures. However, the authors limited themselves to minimum factors to estimate the energy consumption.

Though many engineering models were proposed and developed, unfortunately none of them were validated to account for accuracy. Though the statistical models validated their model, they require extensive historical data.

\subsection{Conclusion}

This chapter initially explains and presents the studies on the factors which affect the energy consumption of the HVAC systems. Then it presents the models available in the market and the new models proposed by the researchers to estimate the energy consumption of HVAC system. The models present in the market require extensive data. The models proposed by the researchers require minimal data but do not consider all the factors. To overcome these limitations this research proposes a new user-friendly model to estimate the energy consumed by HVAC system based on the five factors mentioned Occupancy, Weather, Volume of the Room, Building Envelope and Thermostat set point. 


\section{Chapter 3 : Methodology}

\subsection{Problem Statement}

With million residential houses being built every year [27], It is important to know the effect of various factors on the HVAC energy consumption. Though there has been significant research done on estimating energy usage and optimizing it, most of the studies overlooked the combined effect of all the factors that affect the energy consumption. In the residential sector, the lack of knowledge among the users on the effect of the factors like insulation, thermostat level, zoning etc.; this may lead to a lot of investment on the HVAC systems and the building envelope which may or may not be economically feasible.

The main objective of this research is to develop a simulation model which will allow the users to estimate the total energy consumption of a HVAC system with smart thermostat based BEMS in a residential house. Thus, it provides an opportunity to the user to decide on purchasing the HVAC system and the controls associated. The major factors which affect the HVAC consumption are

- Occupancy

- Thermostat

- Volume of the individual rooms

- Weather

- Building envelope 


\subsection{Description}

The simulation is done in Microsoft Excel® based on the discrete event Monte Carlo Simulation. The randomness of the variables is generated using the heuristic approach.

\subsubsection{Monte Carlo Simulation}

Monte Carlo Simulation is a method that is used to find solutions to mathematical and statistical problems through statistical sampling. The results are computed based on random sampling and statistical analysis. The main purpose of the Monte Carlo simulation is to understand the impact of risk and uncertainty of a particular system. The behavior of the systems can be observed by the experimental process of drawing many samples and then analyzing their behavior. Though we have ample information about the factors affecting the outcome, it is difficult to predict the future with minimum error. Monte Carlo Simulation generates a possible outcome of a decision, thereby helping us to take a better decision under uncertainty. The simulations help to build a model by substituting various values for any factor that has uncertainty based on probability distribution. It records the results and continues to perform the same calculations repeatedly. It uses a different set of random variables from the distribution every time. To generate different sets of random variables continuously, it requires programming or software help. This study utilizes Microsoft Excel@ VBA® (Visual Basic for Applications) to perform the simulation.

\subsubsection{Heuristic Approach}

Heuristics are simple and efficient guidelines that people often use to make conclusions. It is a method of problem-solving, learning or discovery that employs a practical method, which may or may not provide optimal solutions but is sufficient for immediate goals. 


\subsubsection{VBA}

Visual Basic Application (VBA) is a programming language foe Excel® and other Microsoft Office ${ }^{\circledR}$ programs. It enables building user-defined functions and automating processes. VBA allows users to automate by writing code or by simply creating macros. Excel VBA is employed to code the excel model, to perform the calculations and run the simulation model. The visual basic code used in this study is shown in the Appendix.

\subsection{Definitions}

The HVAC discipline measures the capacity of heating and cooling systems with many different terms. Some of the important ones are

\section{BTUH (British Thermal Units per Hour)}

HVAC units are usually measured often in terms of BTUH or Tons of HVAC. A ton of HVAC has the ability to remove 12,000 BTUs per hour.

$$
1 \text { Ton }=12,000 \mathrm{BTU} / \mathrm{hr}
$$

The energy consumption by the system can be calculated using the formulae

$$
\begin{aligned}
& \text { Energy consumption }=\mathrm{R} \times \mathrm{LF} \times \mathrm{UF} \times \mathrm{OH} \\
& \mathrm{R}=\quad \text { Rating of the System }(\mathrm{Btu} / \mathrm{hr}) \\
& \mathrm{LF}=\quad \text { Load Factor }(\%) \\
& \mathrm{UF}= \\
& \mathrm{OH}=\quad \text { Otilization Factor }(\%)
\end{aligned}
$$




\section{Load Factor}

The ratio of the load that equipment draws under operating conditions to the load that it could draw at maximum capacity.

\section{Utilization Factor}

The ratio of time that equipment is used to the total time it could be used.

\section{Coefficient of Performance (COP)}

$\mathrm{COP}$ is defined as the ratio of the useful cooling provided to work required. It can also be stated as ratio of BTUs of heat removed to the wattage of energy consumed by the system.

\section{Heating Seasonal Performance Factor (HSPF)}

HSPF is defined as the ratio of output heating provided to the input electrical energy. It is measurement of efficiency of a system and the units are BTU/Wh.

\subsection{Theory}

As the energy consumption is a function of the four factors mentioned above (rating, load factor, utilization factor, and operating hours), we need to determine each of these factors. However, the HVAC system rating is one of the inputs given by the user. As the simulation is done with a frequency of 30 minutes for a whole year, utilization factor and the operating hours are taken care of. So, the main goal of the model is to determine the load factor. As mentioned earlier, the load factor of the system is adjusted based on five factors. The process of determination of the load factor is shown in the upcoming section 3.7. 


\subsection{Software Model}

The title of the model developed is "Smart Thermostat based BEMS." The model was developed using Visual Basic Applications (VBA) in Microsoft Excel. The model was created with a user-friendly interface while maintaining high degree of detail.

\subsubsection{User Inputs and Model Outputs}

One of the inputs which user needs to provide is the configuration of the house mainly the number of bedrooms. The simulation considers one kitchen and a living room along with the bedrooms.

The model also requests the user if there are any plans for building envelope improvements and asks for the month in which improvements are done if any. The model also asks for the number of times the thermostat settings are changed.

The user also has the privilege to input the volume, building envelope and the thermostat levels. However, if the user fails to input these values, the model generates a random level of all the three factors. The rules behind selecting the random number are explained in the corresponding sections.

The model also checks if there are any power outages in the area. If any, the user needs to provide the maximum number of power outages and the maximum time one power outage may last.

The simulation model allows the user to input the capacity of the HVAC system and rate structure associated. This allows the model to calculate the energy consumption and the associated costs to run the HVAC systems. The energy sources considered are Natural Gas 
and Electricity. As many residential areas are not subjected to demand costs, this study does not consider any peak demand or any costs associated with demand.

The model also considers the location where the user's house is located as the weather varies all over the United States. The user can select from nine census regions as explained in section 3.8. The user is guided with a map and region number for information, which can be helpful to determine his or her region.

After the user provides all the inputs, the model runs the simulation. The completed simulation then provides an estimate of the energy consumption and the energy costs that are generated based on the inputs provided by the user.

\subsection{Factors}

\subsubsection{Occupancy}

Occupancy is an important factor which affects the energy efficiency of HVAC systems. Employing a thermostat which takes occupancy as one of the feedbacks, can have a great impact on the energy consumption. This study calculates the energy consumption with an assumption that each room has a smart thermostat with occupancy detecting capability. These can sense if the room is occupied or not and set back the thermostat when the room is unoccupied. The occupant's schedule and some heuristic rules are used to sense the occupancy of a person in the room. The model is flexible. If the user of the model feels that the schedule does not match the base schedule used for the simulation, he or she can change the schedule accordingly. The model has the capability to differentiate between a weekday and a weekend and change the heuristic rules accordingly. The rules used are shown in the table below. The room occupancy probabilities are not same; they vary as per the time of 
the day. They also change based on the type of the room and time of the day. For example, from the table 3.1 shown below, it can be observed that a bedroom has a probability of $85 \%$ to be occupied between 12:00 am and 8:00 am, however, at the same time the living room has a probability of only $20 \%$. As the frequency of the model is for 30 minutes, for each step, i.e., for every 30 minutes, occupancy is sensed for all the rooms and occupancy level is established. However, the model estimates the time of sleep and sleeping time of the user and retains the occupancy level until he or she sleeps and then the occupancy level continues to update.

Table 3.1: Rules to determine Occupancy

\begin{tabular}{|c|c|c|c|}
\hline \multicolumn{4}{|c|}{ Bed Rooms } \\
\hline Start Time & End Time & Weekday & Weekend \\
\hline 11:00 PM & 9:00 AM & $85 \%$ & $85 \%$ \\
\hline 9:00 AM & $5: 00 \mathrm{PM}$ & $15 \%$ & $50 \%$ \\
\hline 5:00 PM & $11: 00 \mathrm{PM}$ & $50 \%$ & $50 \%$ \\
\hline
\end{tabular}

\begin{tabular}{|c|c|c|c|}
\hline \multicolumn{4}{|c|}{ Living Room } \\
\hline Start Time & End Time & Weekday & Weekend \\
\hline 11:00 PM & 9:00 AM & $20 \%$ & $20 \%$ \\
\hline 9:00 AM & $5: 00$ PM & $40 \%$ & $70 \%$ \\
\hline 5:00 PM & $11: 00$ PM & $60 \%$ & $60 \%$ \\
\hline
\end{tabular}

\begin{tabular}{|c|c|c|c|}
\hline \multicolumn{4}{|c|}{ Kitchen } \\
\hline Start Time & End Time & Weekday & Weekend \\
\hline 11:00 PM & $6: 00 \mathrm{AM}$ & $10 \%$ & $10 \%$ \\
\hline 6:00 AM & $2: 00 \mathrm{PM}$ & $65 \%$ & $65 \%$ \\
\hline 2:00 PM & $5: 00 \mathrm{PM}$ & $40 \%$ & $40 \%$ \\
\hline 5:00 PM & $11: 00 \mathrm{PM}$ & $75 \%$ & $75 \%$ \\
\hline
\end{tabular}

\begin{tabular}{|c|c|c|c|}
\hline \multicolumn{4}{|c|}{ Bathroom } \\
\hline Start Time & End Time & Weekday & Weekend \\
\hline 12:00 AM & $12: 00 \mathrm{AM}$ & $15 \%$ & $15 \%$ \\
\hline
\end{tabular}

These above rules are developed considering a typical working employee (Leaves to work at 8:00 AM and returns by 5:00 PM). The model is flexible enough; so if the user works at night or disagrees with the above rules, the user can update the rules by changing the percentages in the cells shown below in Sheet "References." 


\begin{tabular}{|c|c|c|c|c|c|c|c|c|c|c|c|c|c|c|}
\hline 4 & A & B & c & $D$ & $E$ & $\mathrm{~F}$ & G & H & 1 & J & k & L & M & $\mathrm{N}$ \\
\hline 1 & \multicolumn{14}{|c|}{ Occupancy } \\
\hline 2 & \multicolumn{4}{|c|}{ Bed Rooms } & & \multicolumn{4}{|c|}{ Hall } & & \multicolumn{4}{|c|}{ Kitchen } \\
\hline 3 & & & Working Days & Holiday & & & & Working Days & Holiday & & & & Working Days & Holiday \\
\hline 4 & $0: 00$ & $8: 30$ & 0.85 & 0.85 & & $0: 00$ & $8: 30$ & 0.2 & 0.2 & & $0: 00$ & $5: 30$ & 0.1 & 0.1 \\
\hline 5 & 9:00 & $16: 30$ & 0.15 & 0.5 & & 9:00 & $16: 30$ & 0.4 & 0.7 & & $6: 00$ & $13: 30$ & 0.65 & 0.65 \\
\hline 6 & $17: 00$ & $22: 30$ & 0.5 & 0.5 & & $17: 00$ & $22: 30$ & 0.6 & 0.6 & & $14: 00$ & $16: 30$ & 0.4 & 0.4 \\
\hline 7 & 23:00 & $0: 00$ & 0.85 & 0.85 & & 23:00 & $0: 00$ & 0.2 & 0.2 & & $17: 00$ & $22: 30$ & 0.75 & 0.75 \\
\hline 8 & & & & & & & & & & & $22: 30$ & 0 & 0.1 & 0.1 \\
\hline
\end{tabular}

\section{Figure 3.1: Rules for Occupancy - Sheet "References"}

\subsubsection{Weather}

Weather and climatic conditions like outside temperature, humidity and moisture are the other main factors to be considered. In this study, weather is assumed to be at three levels - level 1 as mild, level 2 as moderate and level 3 as extreme weather conditions. The weather factor is updated for every eight hours and is assumed to be same for the next eight hours. To determine the weather factor, the year is split into four seasons and the rules are shown below.

\section{Table 3.2: Rules to determine Weather Factor}

\begin{tabular}{|c|c|c|c|c|}
\hline Months & Season & level 1 & level 2 & level 3 \\
\hline March 1st - May 31st & Spring & $40 \%$ & $40 \%$ & $20 \%$ \\
\hline June 1st - August 31st & Summer & $40 \%$ & $35 \%$ & $25 \%$ \\
\hline September 1st - November 1st & Fall & $40 \%$ & $40 \%$ & $20 \%$ \\
\hline December 1st - February 28th & Winter & $10 \%$ & $30 \%$ & $60 \%$ \\
\hline
\end{tabular}

It should be noted that the climate conditions vary as per location. The United States is divided into nine regions as shown in the figure 3.2. This study considers all these regions to evaluate the energy consumption. The model uses heating and cooling degree days to determine if heating or cooling is required. Section 3.8 explains how the degree days are used to determine the energy consumption based on the region. 


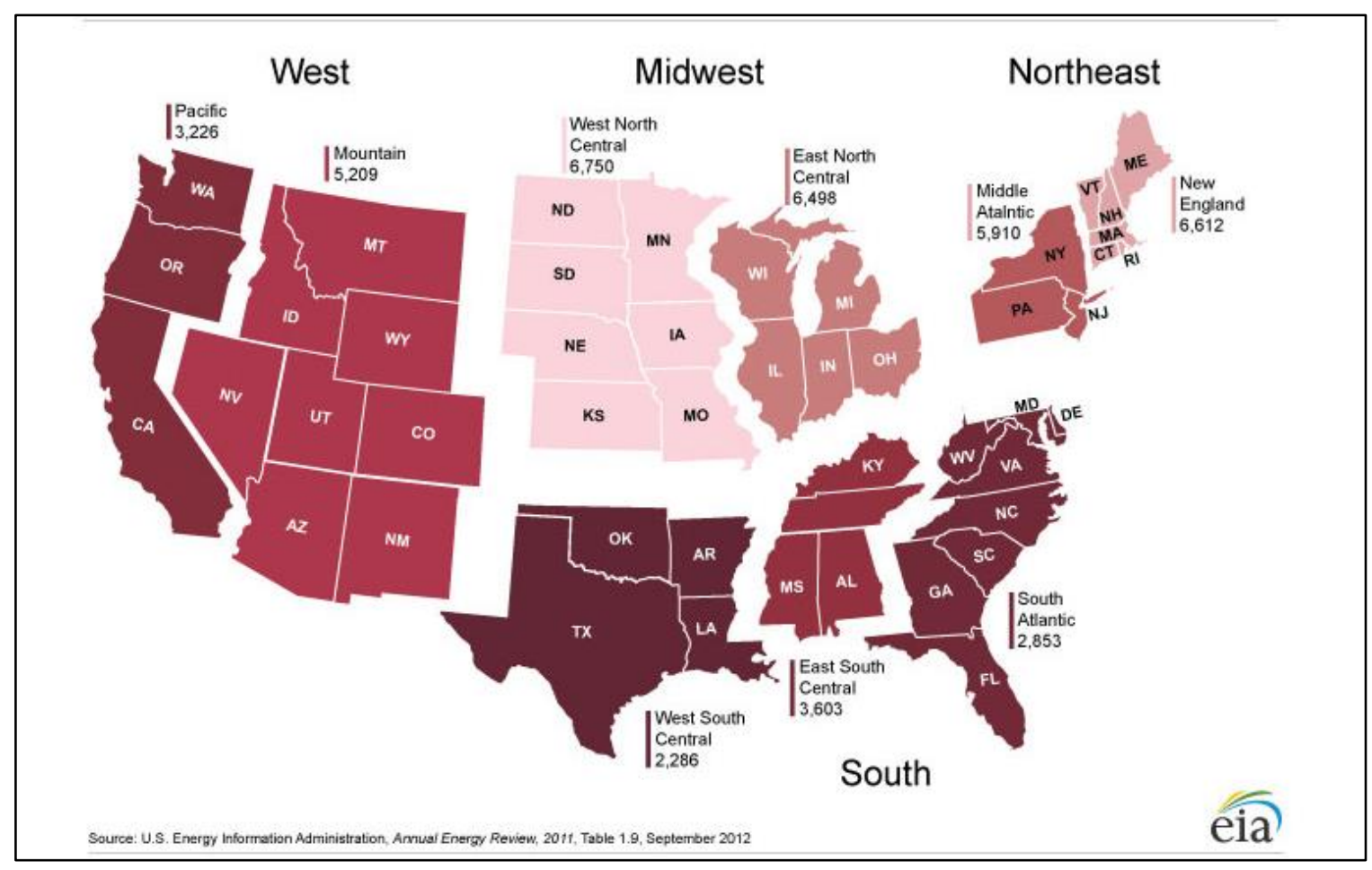

Figure 3.2: Census Regions [28]

\subsubsection{Volume of the Room}

To lower or increase the room temperature, the HVAC should either add or remove heat from the area. The amount of heat or cold air added to the room is directly proportional to the volume of the room. Three levels are considered for volume of the room - level 1 as small, level 2 as medium and level 3 as large. However, this is an input from the user. The user can decide the level for that room from the reference table provided in the model. The level is randomly generated if the user does not provide input. The randomness is generated assuming that all the three levels have an equal probability of $33 \%$. As the volume of the room is directly proportional to the square footage of the room, the areas of the room are considered or provided for the user to determine the volume level. The table 3.3 below shows the standard floor area of different rooms in residential buildings. 
Table 3.3: Standard Sizes of rooms in Residential Building[29]

\begin{tabular}{|c|c|c|c|c|}
\hline \multicolumn{5}{|c|}{ Floor Area (Sq.ft) } \\
\hline Level & Bedrooms & Bathroom & Living Room & Kitchen \\
\hline 1 & 144 & 54 & 216 & 50 \\
\hline 2 & 250 & 70 & 320 & 104 \\
\hline 3 & 384 & 96 & 616 & 120 \\
\hline
\end{tabular}

Table 3.4: Rules to determine volume level

\begin{tabular}{|c|c|c|c|}
\hline & Level 1 & Level 2 & Level 3 \\
\hline Volume & $33.3 \%$ & $33.3 \%$ & $33.3 \%$ \\
\hline
\end{tabular}

\subsubsection{Building Envelope}

A physical separator between the exteriors and the interior of a building is called building envelope. Building envelope includes walls, fenestration, roofs and doors. Insulation in the walls and the roof is a barrier to heat loss and heat gain. The arrangement of the windows, type of windows (i.e., single pane or double pane, with or without low emissivity coating) and their orientation respective to the building can easily add cooling or heating loads to the building. Thus, the building envelope is considered as one of the critical factors to determine the load factor. Two levels - level 1 as good and level 2 as bad are considered for the building envelope. The level for building envelope is an input given by the user. However, if the user fails to input the level for the building envelope it is generated randomly. For the study, two levels are considered for the building envelope and are distributed at $50 \%$ each. The model also asks the user if there are any plans to upgrade the building envelope and if there are any, it also asks for the month when the upgrades are planned. The model updates the building envelope level from that month. 
Table 3.5: Rules to determine Building Envelope

\begin{tabular}{|c|c|c|}
\hline & Level 1 & Level 2 \\
\hline Building Envelope & $50 \%$ & $50 \%$ \\
\hline
\end{tabular}

\subsubsection{Thermostat Setpoint}

People prefer their comfort levels, i.e., one might prefer their room at a higher temperature and the others might prefer their room at lower temperatures. As mentioned, the thermostat setpoint can affect the energy consumption. This is also another input from the user. Like the volume and building envelope, if the user does not want to enter the level, it is randomly generated. There are two levels of thermostat factor - level 1 for lower set point and level 2 for higher set point. The tables below help the user and the model to determine the thermostat level respectively. The statistics shown below in table 3.6 are the outputs from a survey [30]. The model also considers the number of times the thermostat settings are changed yearly. Then it updates the thermostat level with the updated frequency (365/number of changes per year). However, if there are no changes to the thermostat, the initial input from the user will be used for the calculations.

Table 3.6: User Guidelines to determine the level for the thermostat

\begin{tabular}{|c|c|c|}
\hline & Level 1 & Level 2 \\
\hline Summer & $70-80^{\circ} \mathrm{F}$ & $60-70^{\circ} \mathrm{F}$ \\
\hline Winter & $60-70^{\circ} \mathrm{F}$ & $70-80^{\circ} \mathrm{F}$ \\
\hline
\end{tabular}

Table 3.7: Model Guidelines to determine the level for the thermostat

\begin{tabular}{|c|c|c|}
\hline & Level 1 & Level 2 \\
\hline Summer & $68 \%$ & $32 \%$ \\
\hline Winter & $37 \%$ & $63 \%$ \\
\hline
\end{tabular}




\subsection{Load Factor Determination}

Load factor is a function of the five factors - occupancy, outside weather, thermostat set point, building envelope and volume of the room. Thus, assigning levels for all the five factors is a pre-requisite to determining load factor. There are two levels for occupancy, three levels for weather, three levels for volume of the room, two levels for building envelope and two levels for thermostat set point. Thus, the maximum number of combinations possible considering all these levels and factors are 72 sets $(2 \times 3 \times 3 \times 2 \times$ 2). Load factor is assigned to each possible set (pre-determined).

To determine the load factors for each of the 72 pre-determined sets, worst conditions are considered and HVAC system is assumed to be running at full load under these conditions. Then the load factors are assigned to the remaining 71 sets. Example for determination of load factor for six sets is shown below in table 3.8.

Table 3.8: Load Factor Assignment

\begin{tabular}{|c|c|c|c|c|c|c|}
\hline Category & Case 1 & Case 2 & Case 3 & Case 4 & Case 5 & Case 6 \\
\hline Load Factor & $100 \%$ & $40 \%$ & $80 \%$ & $70 \%$ & $20 \%$ & 0 \\
\hline Occupancy & 1 & 0 & 1 & 1 & 1 & 0 \\
\hline Weather & 3 & 3 & 3 & 3 & 1 & 1 \\
\hline Volume of the Room & 3 & 3 & 2 & 2 & 1 & 1 \\
\hline Building Envelope & 2 & 2 & 2 & 1 & 1 & 1 \\
\hline Thermostat Set Point & 2 & 2 & 2 & 2 & 1 & 1 \\
\hline
\end{tabular}

In case 1 , there is a large room which is occupied by the user, the room has a poor building envelope, the outside weather is severe and user prefers high comfort levels (above 70 in winter and below 70 in summer). These are the worst conditions possible that can put a maximum load on the HVAC system. So considering these conditions, HVAC will run at full load. In case 2, all the levels are same as case 1 except for the fact that the room is unoccupied. In this scenario, the thermostat sense that the room is unoccupied and sets 
back the temperature. Though the system sets back the temperature, as the building envelope is poor, there would be a lot of infiltration taking place. Thus HVAC systems need to make up for the heat loss or heat gain; so a load factor of $40 \%$ is assigned to the system. In the case 5, the room volume is small, outside weather conditions are mild, the building envelope is good, the user prefers low comfort levels, and is occupied. In these conditions, though there would not be much cooling or heating required, the HVAC system is responsible for circulating the air. Thus a minimum load factor of $20 \%$ is assigned. In case 6 , all the levels are same as case 5 except for the room is unoccupied. As the building insulation is good and the volume is small, there would not be much requirement of heat to be added or removed. Thus, it is assumed that the HVAC system is turned off in these conditions. With the worst (case 1, case 2) and best (case 5, case 6) conditions being assigned, the other sets are also assigned. For example, in case 3 , all the levels are same as case 1 except for the volume of the room is at level 2. In these conditions a load factor of $80 \%$ is being assigned. If the building envelope of the room is improved, case 3 turns to case 4, i.e., there would be less infiltration taking place, thus a lower load on HVAC system. Hence, a load factor of $70 \%$ is assigned.

As the frequency of the simulation is 30 minutes, the generated set (5 factors) is compared to the pre-determined set and a load factor is assigned to that set. Once the load factors are obtained for each room, then a weighted average of these load factors are evaluated with weights being the floor area of the rooms. Then these load factors are used to determine the energy consumption of the HVAC system. Detailed explanation with each step and screen shots is shown in section 4.1. 


\subsection{Energy Costs Calculations}

The capacity of the heater, tonnage and $\mathrm{COP}$ of the air conditioner, type of fuel and the rate structure are inputs given by the user. $\mathrm{kW}$ of the air conditioner is calculated using the COP and tonnage of the air conditioner. The capacity of the heater and $\mathrm{kW}$ of the air conditioner are used while calculating the energy required to heat and cool the building respectively.

The model has the capability of identifying if cooling or heating is required. Thus, the model also considers if there are any requirements for cooling in winter or heating in summer. The model uses degree days of the respective region selected by the user. It then establishes a probability of cooling using the degree heating days and degree cooling days. The table 4.9 below shows the heating degree days and cooling degree days of the region 8 - South Atlantic. The probability of cooling is calculated for that region. For example, the probability for cooling in this region in January is about 5\% while the remaining is heating. It can be observed that the probability of cooling in June, July, and August is $100 \%$, which implies that there is no requirement for heating at all in these months.

Table 3.9: Probability of Air Conditioning

\begin{tabular}{|c|c|c|c|}
\hline Name & HDD & CDD & Cooling \\
\hline January & 569 & 29 & $5 \%$ \\
\hline February & 460 & 30 & $7 \%$ \\
\hline March & 322 & 53 & $15 \%$ \\
\hline April & 129 & 84 & $40 \%$ \\
\hline May & 39 & 189 & $83 \%$ \\
\hline June & 2 & 338 & $100 \%$ \\
\hline July & 1 & 412 & $100 \%$ \\
\hline August & 1 & 392 & $100 \%$ \\
\hline September & 11 & 264 & $96 \%$ \\
\hline October & 118 & 123 & $52 \%$ \\
\hline November & 315 & 47 & $13 \%$ \\
\hline December & 445 & 44 & $9 \%$ \\
\hline
\end{tabular}


The model uses these probabilities to differentiate between cooling and heating. Once the model identifies the difference, energy is calculated for the heater and air conditioner using the formula shown below.

$$
\begin{array}{ll}
\mathrm{kWh} & =\mathrm{kW} \times 0.5 \times \mathrm{LF} \\
\mathrm{MMBtu} & =\mathrm{MMBtu} / \mathrm{hr} \times 0.5 \times \mathrm{LF} \\
\mathrm{kWh} \text { Cost } & =\mathrm{kWh} \times \$ / \mathrm{kWh} \\
\text { MMBtu Cost } & =\text { MMBtu } \times \$ / \mathrm{MMBtu}
\end{array}
$$

Where,

$\mathrm{kW} \quad=$ kilo Watt of Air Conditioner or Heater

$\mathrm{MMBtu} / \mathrm{hr}=$ Capacity of the heater

LF $\quad=$ Load factor of the system

The formula contains 0.5 , as the energy consumption and energy costs are calculated on a 30-minute interval. Once the energy is calculated for all the 30-minute intervals, the energy for the heater and air conditioner are summed up on a monthly basis and on an annual basis. The monthly energy data is printed on a new sheet whereas the total annual consumption pops up in the message box.

\subsection{Assumptions and Limitations}

The energy consumption calculations have been performed by making some assumptions in the model. The model also has some limitations. The assumptions and model limitations are shown below. 


\section{$\underline{\text { Assumptions: }}$}

1. The frequency of the simulation considered for the study is 30 minutes. The model may not be able to react to any changes which may happen between 0 and 29 minutes. It assumes that the levels are assigned, considering the average conditions of the factors in that 30-minute interval.

2. The load factor determined would be the average load factor for the 30-minute interval.

3. The Building envelope improvements are assumed to be done on the first day of the month. Thus the level is updated on the first day of the month.

4. The simulation run is for a typical working year, i.e., no natural disasters are considered.

5. The model assumes that there is a smart thermostat with occupancy detection capability in each room -bedroom, kitchen and living room.

6. The model assumes that the HVAC System are zoned with Variable Air Volume (VAV) controls

7. The model assumes that the bathrooms are tied up with the respective bedrooms.

8. The model do not consider any affects because of ventilation and exhaust air.

9. Weather Factor only updates after 8 hours.

10. The occupancy of the room does not change when the occupant in the bedroom sleep.

11. The demand costs are not considered for estimating the total energy costs.

\section{$\underline{\text { Limitations }}$}

1. The maximum number of bedrooms the model can handle is five. 
2. Natural gas and Electricity are the only energy sources considered for the model.

3. The scope of the study does not include the sizing of HVAC systems. Thus, the total energy consumption is highly dependent on the user to input the correct HVAC system sizes.

\subsection{Conclusions}

This chapter defines the different factors in the model and describes the methodology used to develop the model. In addition, it explains several inputs required to run the simulation. It also addresses the issues a user might encounter if they could not provide all the inputs correctly. Due to the flexibility and user-friendliness, the model can be used by any person to estimate the energy consumption of a residential HVAC system with smart thermostat based BEMS. 


\section{Chapter 4 : System Execution and Results}

This chapter will explain with the help of an example on how the user would go about using the model most effectively. All the inputs will be entered into the program. The cost estimates obtained will be used strictly for the example case and will not remain in the model.

\subsection{Example Input}

For the example case, it is determined that the user's house has two bedrooms, a living room and a kitchen. The HVAC systems employ a natural gas furnace with $30,000 \mathrm{Btu} / \mathrm{hr}$ and 2 Tons air conditioner with COP of 3 for heating and cooling respectively. The user plans to upgrade the building envelope in June. The user updates the thermostat 2 times a year. The user expects a maximum of 2 power outages in his area; each power outage may last for 6 hours. It is assumed that the user's house is in Morgantown (Region 8 - South Atlantic). The rate structure associated with the natural gas and electricity considered are \$7/MMBtu and \$0.1/kWh.

The model has a set of guidelines in sheet "User Manual" as shown in figure 4.1. These are present to provide the user insight into the levels of all the factors considered, procedure the user needs to follow and the limits of the simulation itself. It is important to follow the same procedure mentioned in the manual as one step is dependent on the other. The first step is to clear the past data if there is any. This can be done by using the clear button in the sheet "User Manual." Once the past data is cleared, the user can proceed and start the simulation by clicking Start Simulation button. This opens a message box requesting the user to enter the number of bedrooms. The sample message boxes generated, 
and the input entered by the user is shown in figure 4.2. If the user enters any character or any number out of the range, there will be an error message displayed and model takes the user back to the input box generated initially. Once the number of bedrooms are entered, the model then asks the user if there are any plans to improve the building envelope. If the user has plans and enters the inputs accordingly, then it asks when the user is planning to upgrade the building envelope. Later in the next step, it asks if there are any changes to the thermostat and if any, it asks how frequently the thermostat is changed. 


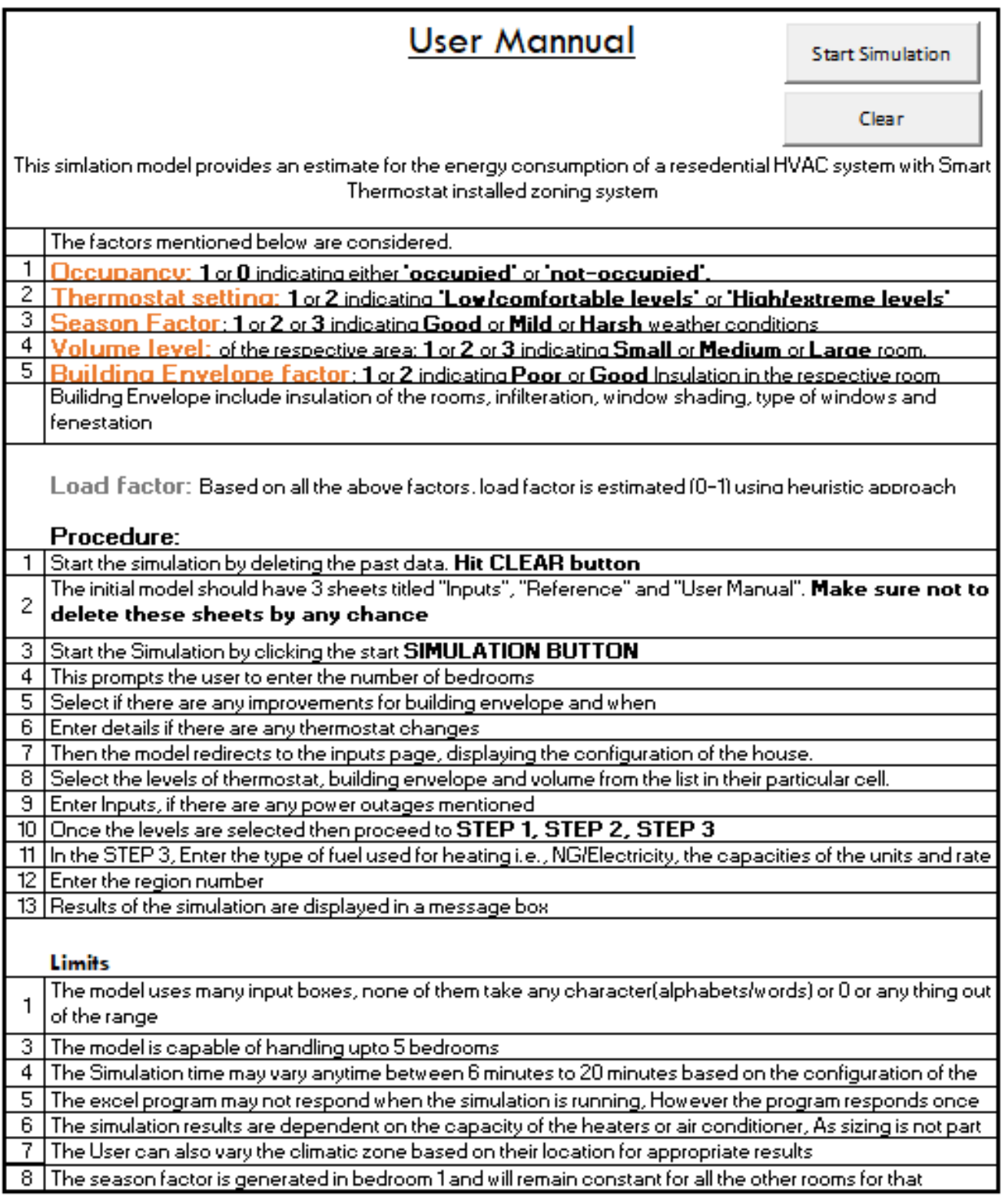

\section{Figure 4.1: User Manual}




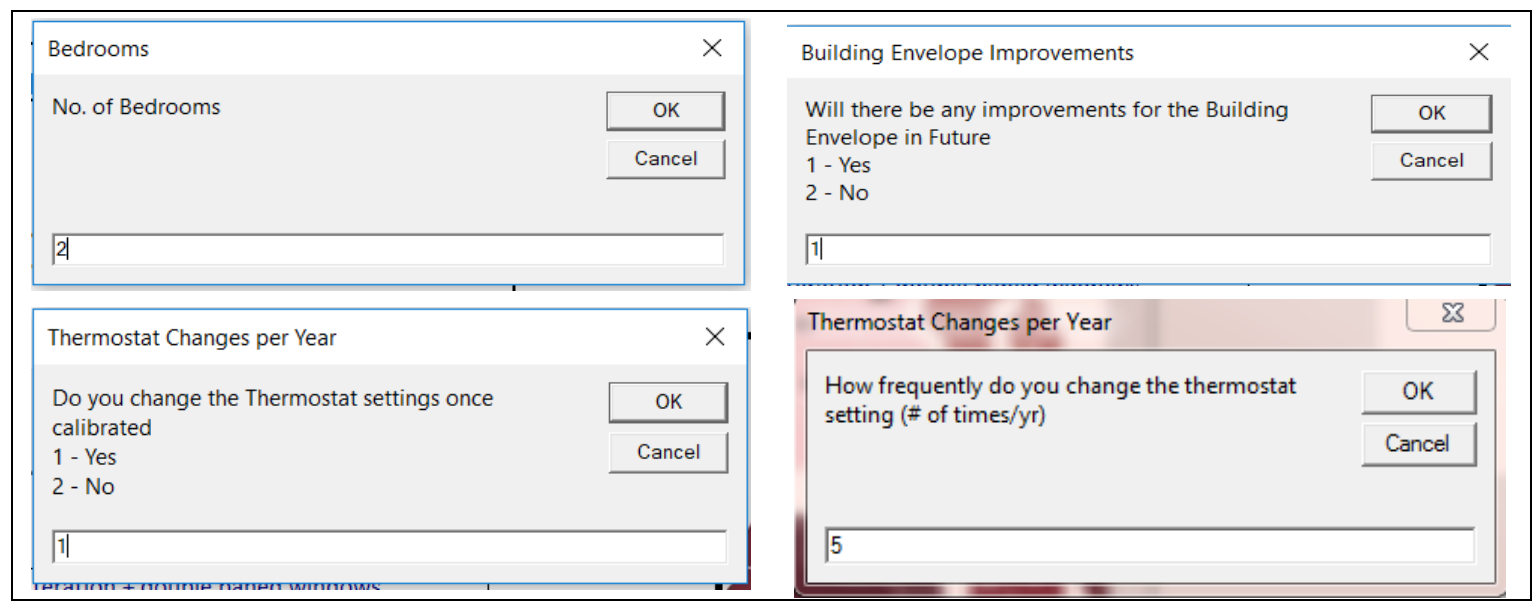

\section{Figure 4.2: Input Boxes seeking Building Specifications}

Next, the model asks if there are any power outages in the area where he resides. Once these inputs are provided, the model directs the user to the sheet "Inputs", where the user must select a level for building envelope, volume of the room and thermostat from the drop-down menu for each room. The model provides an explanation to the user to decide on what level to be selected. If the user mentions any power outages, the user needs to enter the maximum number of power outages expected and the length of the power outage (in hours or days) as shown in figure 4.4. The screenshot of the sheet "Inputs" is shown in figure 4.4 and the drop-down menu is shown in figure 4.3. It should be realized that user can also skip entering the levels. In that case, the levels are generated randomly based on the rules explained in section 4.6.

\begin{tabular}{|l|c|c|c|c|c|}
5 & Room Name & & Building Envelope & Volume & Thermostat \\
\hline 6 & bed room 1 & & 1 & 1 & 2 \\
\hline 7 & bed room 2 & & 2 & 2 & 2 \\
\hline 8 & Living Room & & 2 & 1 & 2 \\
\hline 9 & Kitchen & & 2 & 3 & 2 \\
\hline
\end{tabular}

Figure 4.3: Inputs from User for Factor 


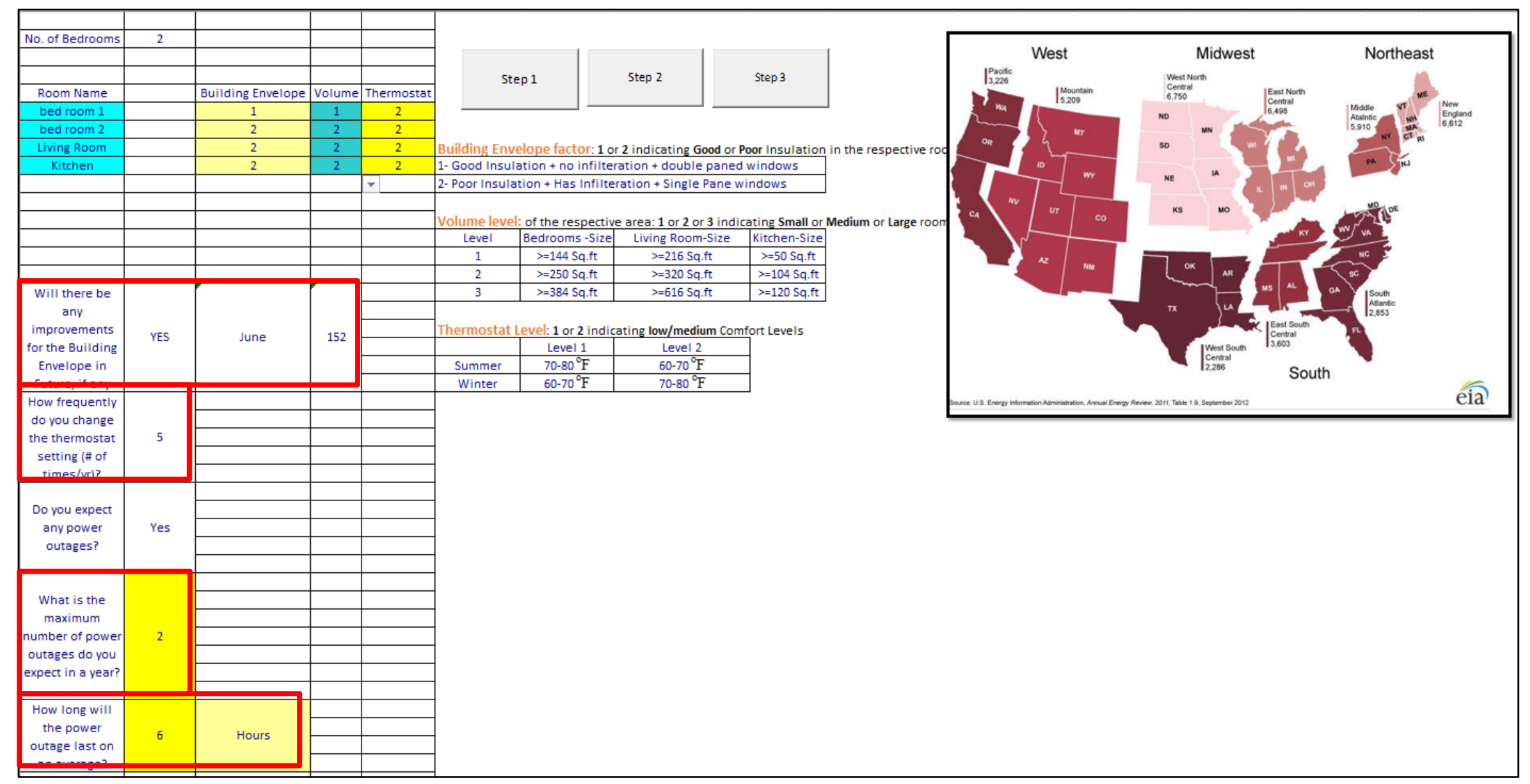

Figure 4.4: Sheet "Inputs" 
With the levels entered, the user now proceeds to the button "Step 1", then four new worksheets (number of bedrooms + Kitchen + Living room) new worksheets are generated with the random numbers for occupancy and weather are generated. Also, levels for the thermostat, building envelope and volume are assigned for each room as per inputs. It should be noted that the random numbers for the weather are same for all the rooms at that particular hour on a particular day, as the weather conditions are same for all the rooms. It is not the case for occupancy. The next step is to determine the levels for the occupancy and the weather factor. As the user clicks the button "Step 2" levels for all the factors occupancy and weather are determined. The user mentioned that there would be improvements to the building envelope in June and mentioned that the user might expect five changes to the thermostat. Thus, the building envelope is updated after June and thermostats are changed five times per year (every 73 days). Step 2 also generates a new worksheet "Load Factor" which consists of the load factors for all the rooms and average load factor for every 30-minute interval as shown in figure 4.5. Once the average load factor is determined, the user clicks the button "Step 3". The user will be prompted to select the location where he stays. The map in the sheet "Inputs" guides the user to select the location. Once the user selects the location, specifications of HVAC system along with the rate structure of the utilities needs to be given. In this scenario, as the user stays in Morgantown, West Virginia which comes under South Atlantic, the user enters 8 in the input box as shown in figure 4.6. In the next input box where the user needs to provide how heating is done (NG / Electricity), the user enters 1. Then user inputs the heating capacity and air conditioning capacity as $0.03 \mathrm{MMBtu}$ and 2 tons, along with the COP of the Air Conditioner. The user then enters the rate structure, i.e., \$/MMBtu and $\$ / \mathrm{kWh}$ as 7 and 0.1 
respectively as shown in figure 4.7. Finally, the simulation runs and displays the energy consumption and the associated costs in a message box as shown in figure 4.8.

\begin{tabular}{|c|c|c|c|c|c|c|c|c|c|c|c|}
\hline 4 & A & B & C & D & E & $\mathrm{F}$ & G & $\mathrm{H}$ & $\mathrm{I}$ & J & K \\
\hline 1 & & & Bedroom1 & Bedroom2 & Living room & Kitchen & Average Load Factor & & & & \\
\hline 2 & \multicolumn{2}{|l|}{ Floor Area } & 144 & 250 & 320 & 104 & & & & & \\
\hline \multicolumn{12}{|l|}{3} \\
\hline \multicolumn{12}{|l|}{4} \\
\hline 5 & Day & Time & Load Factor & Load Factor & Load Factor & Load Factor & Average Load Factor & & & & \\
\hline 6 & 1 & $12: 00 \mathrm{AM}$ & 0.5 & 0.5 & 0.3 & 0.3 & \multicolumn{4}{|c|}{$=$ ROUND(SUMPRODUCT(\$C\$2:\$F\$2,C6:F6)/SUM(\$C\$2:\$F 2$), 2)$} & \\
\hline 7 & 1 & $12: 30 \mathrm{AM}$ & 0.5 & 0.5 & 0.3 & 0.3 & 0.4 & & & & \\
\hline 8 & 1 & $1: 00 \mathrm{AM}$ & 0.5 & 0.5 & 0.3 & 0.3 & 0.4 & & & & \\
\hline 9 & 1 & $1: 30 \mathrm{AM}$ & 0.5 & 0.5 & 0.3 & 0.3 & 0.4 & & & & \\
\hline 10 & 1 & 2:00 AM & 0.5 & 0.5 & 0.3 & 0.3 & 0.4 & & & & \\
\hline 11 & 1 & $2: 30 \mathrm{AM}$ & 0.5 & 0.5 & 0.3 & 0.3 & 0.4 & & & & \\
\hline 12 & 1 & $3: 00 \mathrm{AM}$ & 0.5 & 0.5 & 0.3 & 0.3 & 0.4 & & & & \\
\hline 13 & 1 & $3: 30 \mathrm{AM}$ & 0.5 & 0.5 & 0.3 & 0.3 & 0.4 & & & & \\
\hline 14 & 1 & 4:00 AM & 0.5 & 0.5 & 0.3 & 0.3 & 0.4 & & & & \\
\hline 15 & 1 & 4:30 AM & 0.5 & 0.5 & 0.3 & 0.3 & 0.4 & & & & \\
\hline 16 & 1 & 5:00 AM & 0.5 & 0.5 & 0.3 & 0.3 & 0.4 & & & & \\
\hline 17 & 1 & 5:30 AM & 0.5 & 0.5 & 0.3 & 0.3 & 0.4 & & & & \\
\hline 18 & 1 & 6:00 AM & 0.5 & 0.3 & 0.3 & 0.3 & 0.34 & & & & \\
\hline 19 & 1 & $6: 30 \mathrm{AM}$ & 0.5 & 0.5 & 0.3 & 0.3 & 0.4 & & & & \\
\hline 20 & 1 & 7:00 AM & 0.5 & 0.5 & 0.3 & 0.3 & 0.4 & & & & \\
\hline 21 & 1 & $7: 30 \mathrm{AM}$ & 0.5 & 0.3 & 0.3 & 0.3 & 0.34 & & & & \\
\hline 22 & 1 & $8: 00 \mathrm{AM}$ & 0.6 & 0.8 & 0.35 & 0.35 & 0.53 & & & & \\
\hline 23 & 1 & $8: 30 \mathrm{AM}$ & 0.25 & 0.8 & 0.35 & 0.35 & 0.47 & & & & \\
\hline 24 & 1 & $9: 00 \mathrm{AM}$ & 0.25 & 0.35 & 0.8 & 0.35 & 0.51 & & & & \\
\hline 25 & 1 & 9:30 AM & 0.25 & 0.35 & 0.8 & 0.8 & 0.57 & & & & \\
\hline 26 & 1 & $10: 00 \mathrm{AM}$ & 0.25 & 0.35 & 0.35 & 0.8 & 0.39 & & & & \\
\hline 27 & 1 & $10: 30 \mathrm{AM}$ & 0.25 & 0.35 & 0.35 & 0.35 & 0.33 & & & & \\
\hline 28 & 1 & $11: 00 \mathrm{AM}$ & 0.25 & 0.35 & 0.8 & 0.8 & 0.57 & & & & \\
\hline
\end{tabular}

Figure 4.5: Sheet "Load Factor"

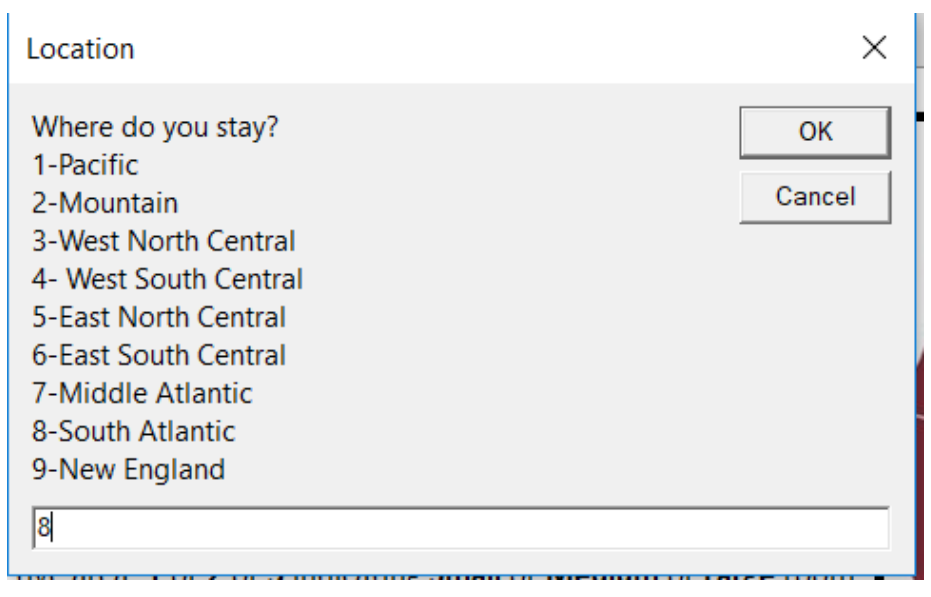

Figure 4.6: Input box for Location 


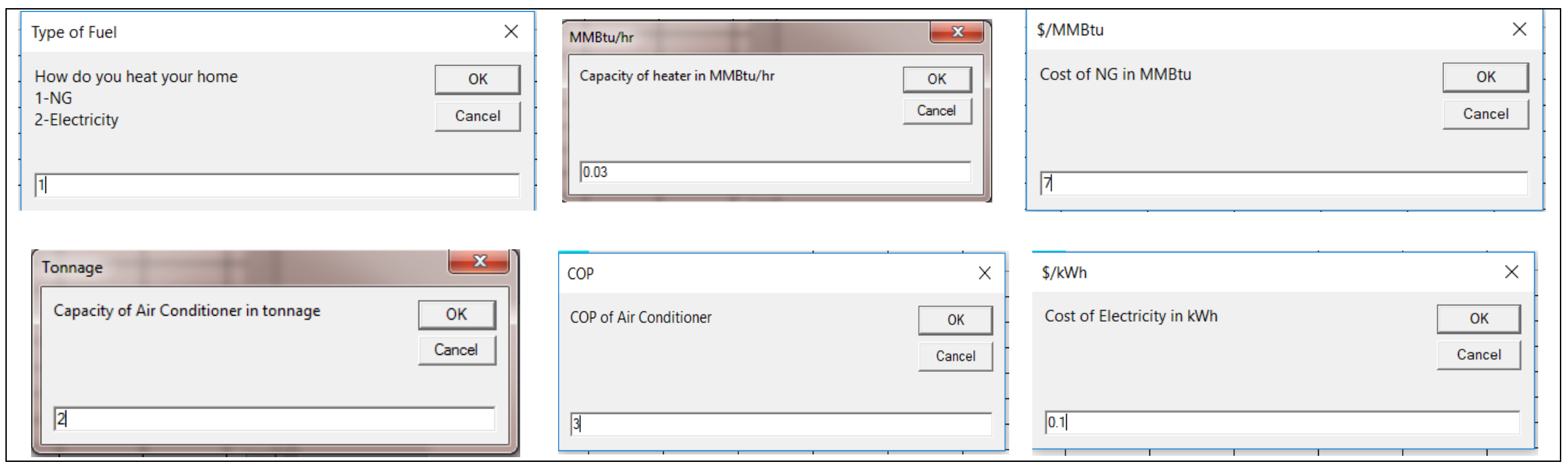

Figure 4.7: Input Boxes for Specifications of HVAC and Rate Structure

\begin{tabular}{|c|c|c|c|c|c|c|c|c|c|c|c|}
\hline & & B & c & D & E & $\mathrm{F}$ & G & $\mathrm{H}$ & & J & k \\
\hline & Date & Time & Average Load Factor & Electricity Consumption & NG Consumption & Electricity costs & NG costs & Total kWh Energy Consumption & | Total MMBtu Energy Consumption & & \\
\hline & & 12:00 AM] & 0.53 & & 0.01 & 0 & 0.07 & 3145 & 54 & & \\
\hline 3 & 1 & 12:30 AM] & 0.53 & & 0.01 & 0 & 0.07 & Total kWh Costs & Total MMBtu Costs & & \\
\hline 4 & & 1:00 AM & 0.53 & & 0.01 & 0 & 0.07 & 315 & 377 & & \\
\hline 5 & 1 & $1: 30 \mathrm{AM}$ & 0.53 & & 0.01 & 0 & 0.07 & & & & \\
\hline 6 & 1 & 2:00 AM & 0.53 & & 0.01 & 0 & 0.07 & & & & \\
\hline 7 & 1 & 2:30 AM & 0.53 & & 0.01 & 0 & 0.07 & & & & \\
\hline 8 & 1 & 3:00 AM & 0.53 & & 0.01 & 0 & 0.07 & & & & \\
\hline 9 & 1 & 3:30 AM & 0.53 & & 0.01 & 0 & 0.07 & & & & \\
\hline & & 4:00 AM [ & 0.53 & & & 0 & & & & & \\
\hline & 1 & 4:30 AM & 0.53 & & 0.01 & $0_{0}$ & 0.07 & & & & \\
\hline 12 & 1 & 5:00 AM & 0.53 & & 0.01 & 0 & 0.07 & & Microsoft Excel & $x$ & \\
\hline & 1 & 5:30 AM & 0.53 & & 0.01 & 0 & 0.07 & & & & \\
\hline 14 & 1 & 6:00 AM & 0.59 & & 0.01 & 0 & 0.07 & & Lation is done $\mathrm{C}$ & & \\
\hline 15 & 1 & 6:30 AM [ & 0.59 & & 0.01 & 0 & 0.07 & & $\begin{array}{l}\text { MMBtu Consumptio } \\
\text { MMtu }\end{array}$ & & \\
\hline 16 & 1 & 7:00 AM | & 0.59 & & 0.01 & 0 & 0.07 & & Electric Consumption $(\mathrm{kWh})=3145 \mathrm{kWW}$ & & \\
\hline & 1 & 7:30 AM & 0.59 & & 0.01 & 0 & 0.07 & & 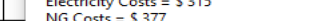 & & \\
\hline 18 & 1 & 8:00 AM [ & 0.42 & & 0.01 & 0 & 0.07 & & & & \\
\hline 19 & 1 & 8:30 AM [ & 0.42 & & 0.01 & 0 & 0.07 & & & & \\
\hline 20 & 1 & 9:00 AM [ & 0.36 & & 0.01 & 0 & 0.07 & & ок & & \\
\hline 21 & 1 & 9:30 AM & 0.28 & & 0 & 0 & 0 & & & & \\
\hline 22[ & 1 & 10:00 AM & 0.31 & & 0 & 0 & 0 & & & & \\
\hline
\end{tabular}

Figure 4.8: Energy Consumption 
The monthly energy consumption and the energy costs associated with the simulation performed are shown in the table below. It can be observed that there is no natural gas consumption in the months of "June, July, August and September" as the user's location does not have any requirement for heating in these months according to the past ten years data. Thus the possibility of heating in these months is very low. It can be observed that there is a possibility for cooling in months "December and January" which are considered as perfect winter months.

Table 4.1: Energy Consumption (Example Input)

\begin{tabular}{|c|c|c|c|c|}
\hline Month & $\begin{array}{c}\text { Electricity } \\
\text { Consumption }\end{array}$ & $\begin{array}{c}\text { Natural Gas } \\
\text { Consumption }\end{array}$ & $\begin{array}{c}\text { Electric } \\
\text { Costs }\end{array}$ & $\begin{array}{c}\text { Natural } \\
\text { Gas Costs }\end{array}$ \\
\hline January & 9 & 12 & 1 & 84 \\
\hline February & 68 & 11 & 7 & 77 \\
\hline March & 35 & 8 & 4 & 56 \\
\hline April & 242 & 5 & 25 & 35 \\
\hline May & 491 & 1 & 50 & 7 \\
\hline June & 470 & 0 & 47 & 0 \\
\hline July & 485 & 0 & 49 & 0 \\
\hline August & 487 & 0 & 49 & 0 \\
\hline September & 483 & 0 & 49 & 0 \\
\hline October & 261 & 2 & 27 & 14 \\
\hline November & 56 & 5 & 6 & 35 \\
\hline December & 54 & 10 & 6 & 70 \\
\hline
\end{tabular}

\subsection{Sensitivity Analysis}

To study the effect of different levels of each factor on energy consumption, simulation runs are performed. The results from the simulation run are plotted and are shown in this section. Though the levels for the factors change for each simulation run, some inputs are considered to be same for each run and they are

- Region : West Virginia - South Atlantic-Region 8

- Zero power outages 
- Capacity of Heater $\quad$ : 50,000 BTU/hr

- Capacity of Air Conditioner : 3 Tons

- COP of Air Conditioner : : 3

The different variables using which we can plot the graphs include:

- Occupancy

- Volume of room

- Thermostat Comfort Levels

- Building Envelope

- Location of the User

\section{Occupancy:}

To observe the effect of energy consumption with and without occupancy detection, a graph is plotted between the monthly energy consumption of HVAC system (Electricity and NG) vs. the occupancy detection capability as shown in figure 4.9 and 4.10 . It is assumed that the regular controls do not have the capacity to detect occupancy. Smart controls have the capability to detect occupancy. Two simulation runs are performed. In the first run all the inputs are entered into the model and simulation is performed. In the second run, simulation is performed to estimate the energy consumption with regular controls. Thus, occupancy level for all the entries (for 24 hours, 365 days) are changed to 1 , keeping all the other factors constant. 


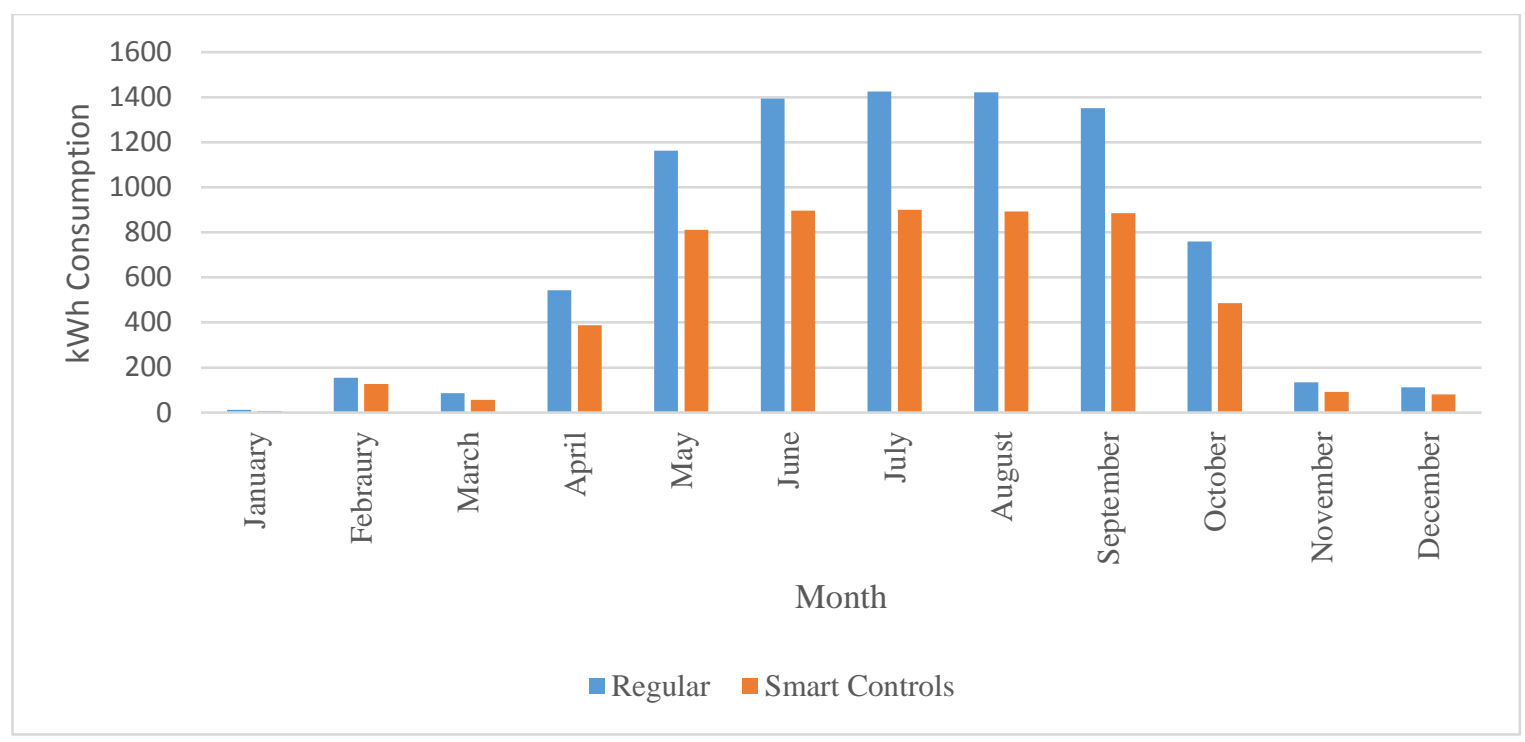

Figure 4.9: Electricity Consumption for Regular Controls vs Smart Controls

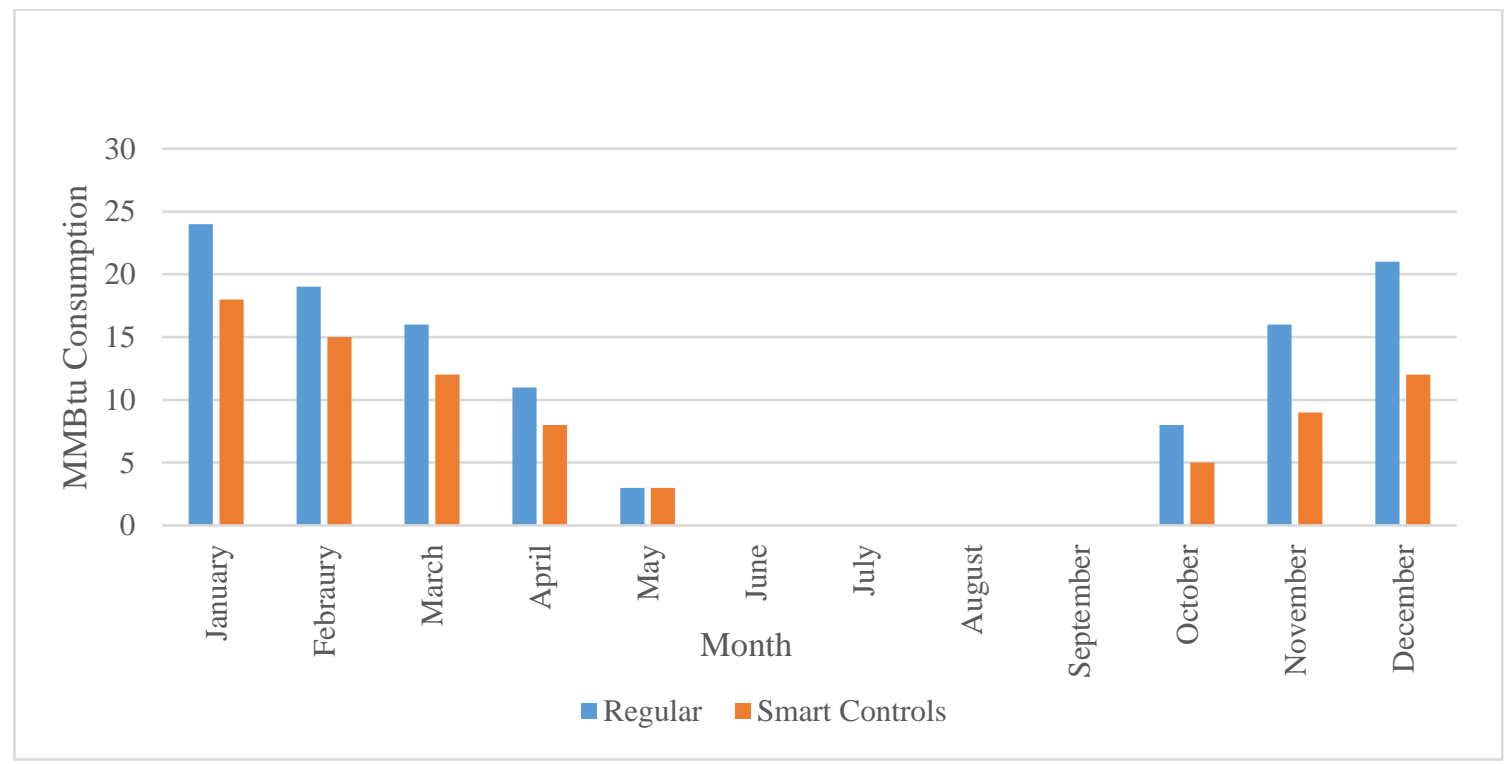

Figure 4.10: NG Consumption for Regular Controls vs. Smart Controls

From the above plot, it is determined that the energy consumption is higher if the occupancy detection is not possible. As the regular controls do not detect occupancy, thus cannot set back the temperature if the room is unoccupied. For the particular simulation run as shown in figure 4.9 and 4.10 , the total energy consumption for a residential house 
with regular controls are about 50\% higher compared to the house employed with smart thermostat based BEMS.

\section{Volume of Room:}

The effect of the volume of room on energy consumption is shown in the figures 4.11 and 4.12. For a particular simulation run, it is assumed that all the rooms are at the same volume level. The volume level is changed in the next run. The three cases used to perform the simulation and obtain the inputs to plot a graph are shown in Table 4.2. It is assumed that there are no building improvement plans and no thermostat changes. A graph is plotted for monthly energy costs vs. volume levels of the room.

Table 4.2: Specifications of Buildings (3 Cases to Plot the Graphs)

\begin{tabular}{|c|c|c|c|c|}
\hline \multirow{4}{*}{ Area } & $\begin{array}{c}\text { Building } \\
\text { Envelope }\end{array}$ & Thermostat & $\begin{array}{c}\text { Volume of the } \\
\text { room }\end{array}$ \\
\hline \multirow{4}{*}{ Case 1 } & Bedroom 1 & 2 & 1 & 1 \\
\cline { 2 - 5 } & Bedroom 2 & 1 & 2 & 1 \\
\cline { 2 - 5 } & Living Room & 2 & 1 & 1 \\
\cline { 2 - 5 } Case 2 & Kitchen & 1 & 2 & 1 \\
\hline & Bedroom 1 & 2 & 2 & 2 \\
\cline { 2 - 5 } & Bedroom 2 & 1 & 1 & 2 \\
\cline { 2 - 5 } & Living Room & 2 & 2 & 2 \\
\cline { 2 - 5 } & Kitchen & 1 & 1 & 3 \\
\cline { 2 - 5 } Case 3 & Bedroom 1 & 2 & 2 & 3 \\
\cline { 2 - 5 } & Bedroom 2 & 1 & 2 & 3 \\
\cline { 2 - 5 } & Living Room & 2 & 1 & 2 \\
\cline { 2 - 5 } & Kitchen & 1 & & \\
\hline
\end{tabular}




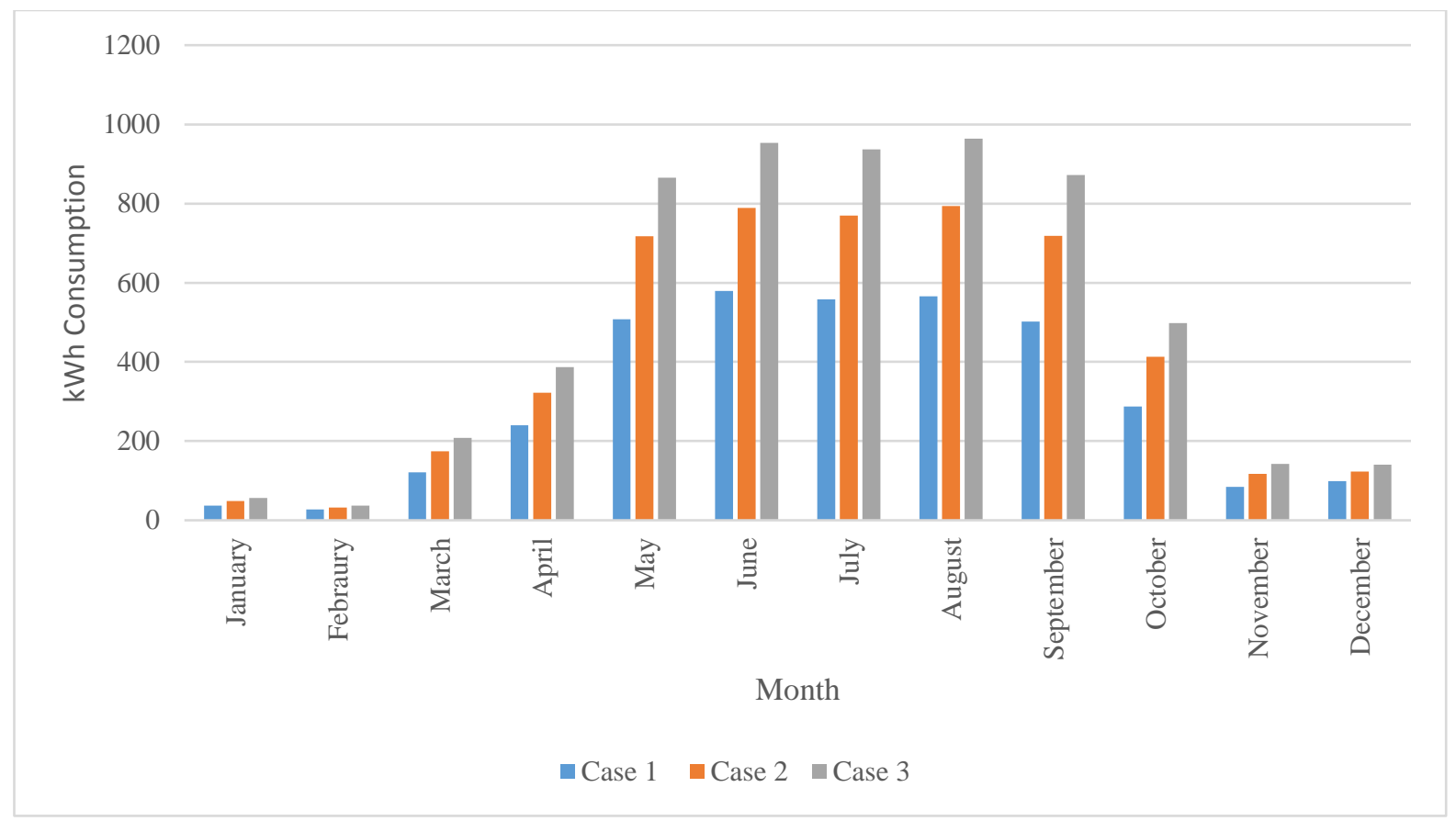

Figure 4.11: Electric Consumption for different volume levels

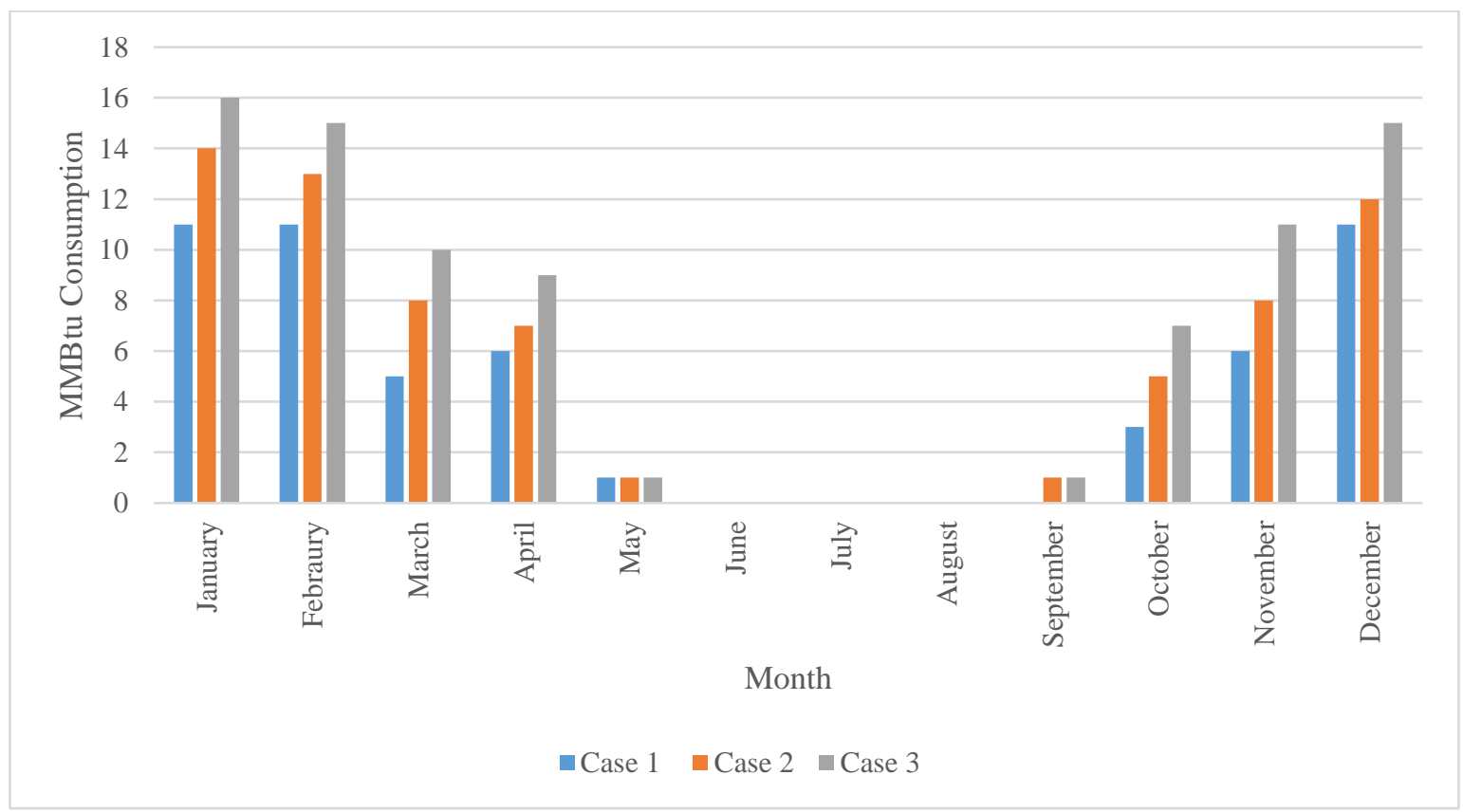

Figure 4.12: NG Consumption for different volume levels

The energy consumption, both natural gas and electricity increase as the volume level increase. The total annual electricity consumption for case 1, 2, and 3 is 3,608, 5,018 and 
$6,059 \mathrm{kWh}$ respectively. The total natural gas consumption for case 1,2 , and 3 is 54,69 and 85 MMBtu.

\section{Thermostat Comfort Levels}

A bar graph is plotted between thermostat comfort levels vs. the energy consumption as shown in figures 4.13 and 4.14 to study the effect of thermostat set points on the energy consumption. Two simulation runs are performed. In the first run (case 1), the thermostat levels are set at level 1 - low comfort levels, while in the second run (case 2), the thermostat levels are set at level 2 - high comfort levels. The energy consumed by the HVAC system is higher in case 2, compared to the energy consumption in case 1.

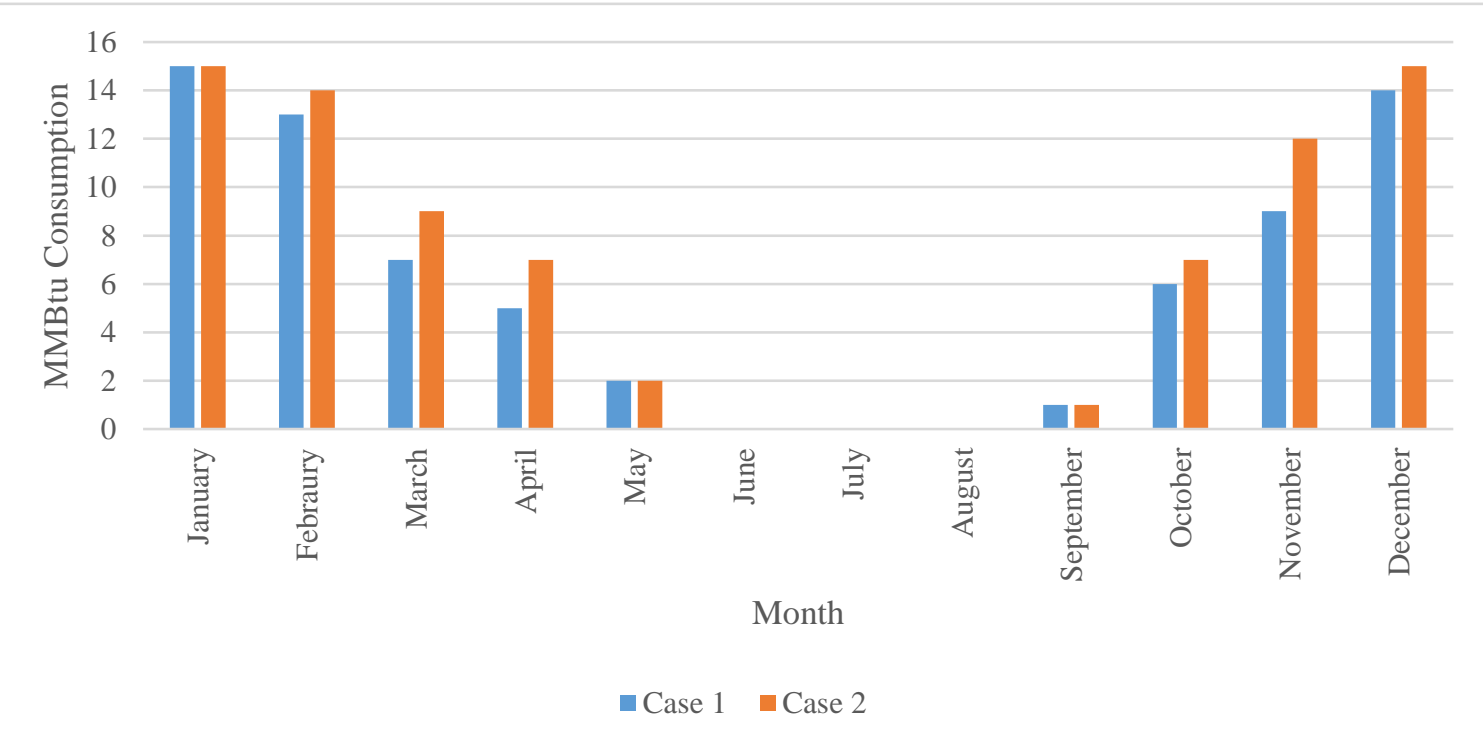

Figure 4.13: Natural Gas Consumption for different Thermostat Comfort levels 


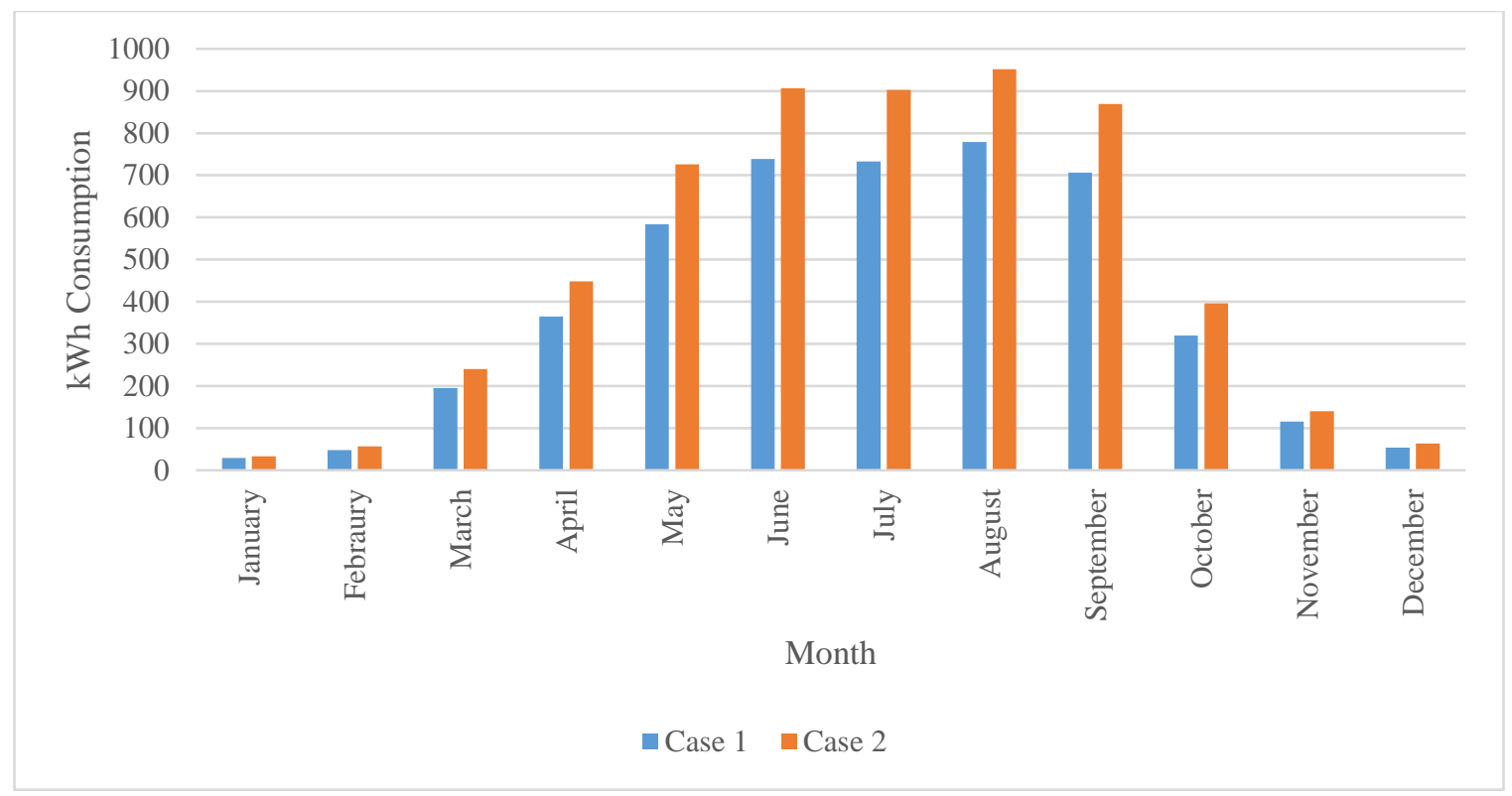

Figure 4.14: Electric Consumption for different Thermostat Comfort levels

The model also considers how many times the thermostat comfort levels are changed. Three different simulation runs are performed to know if there is any relationship between the number of times the thermostat is changed and the energy consumption. The figures 4.15 and 4.16 are bar graphs showing the outputs from the three simulation runs. In the first run (case 3) thermostat set points are changed daily, second run (case 4) they are changed quarterly and in the third run (case 5), they are updated semiannually. 


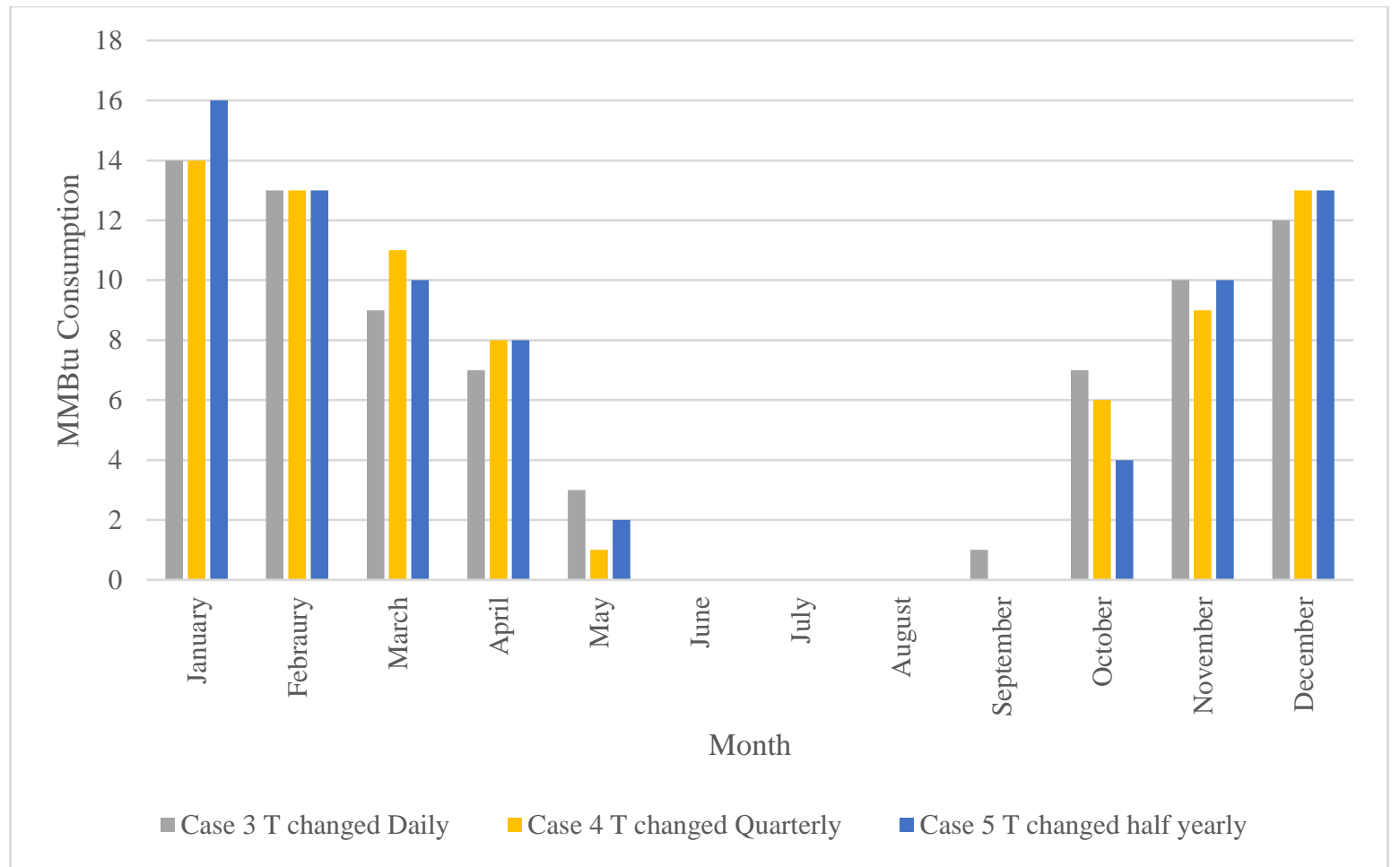

Figure 4.15: Natural Gas Consumption vs. \# of times Thermostat set points changed

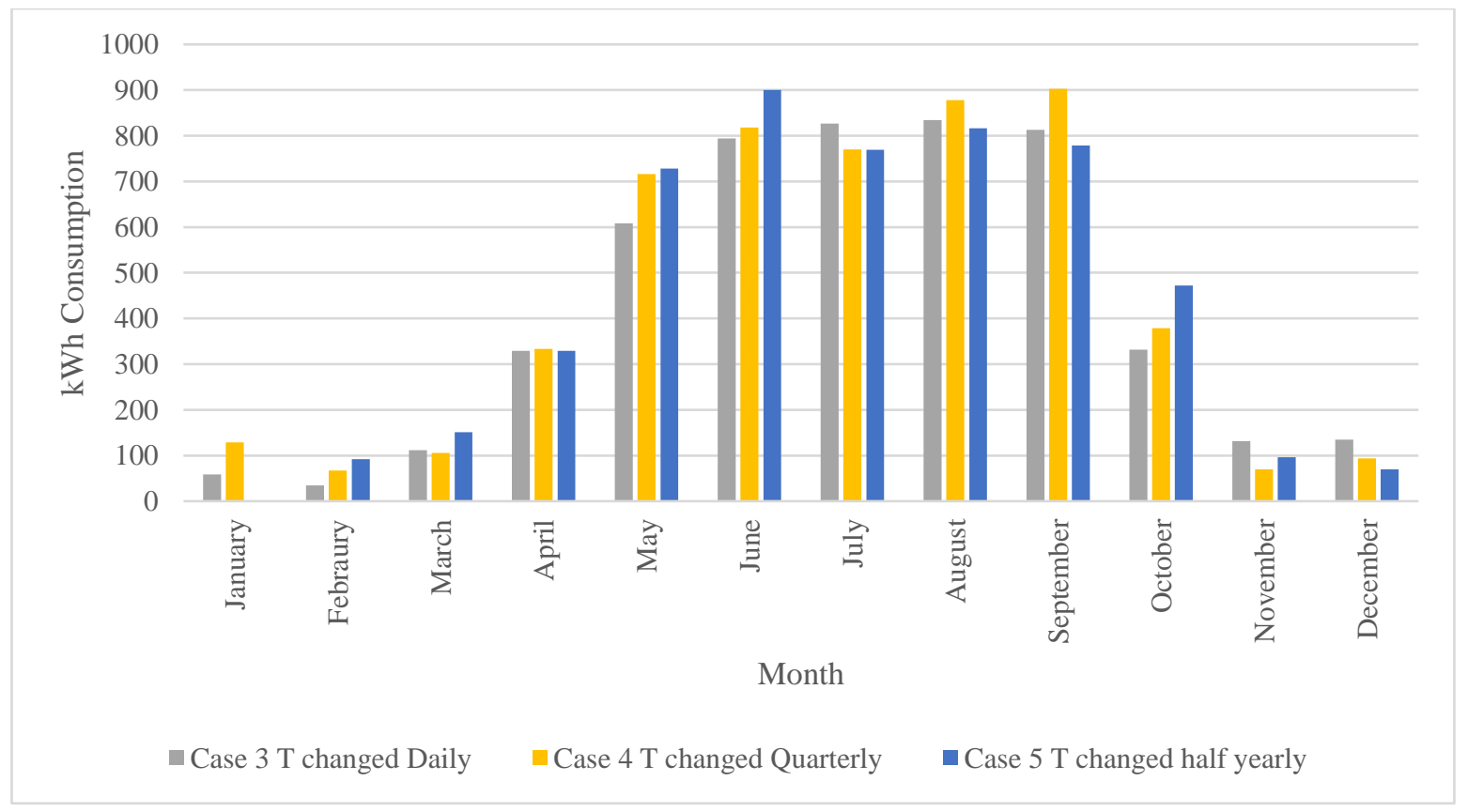

Figure 4.16: Electric Consumption vs. \# of times Thermostat set points changed

It can be observed that the energy consumption does not have any relation with the number of times the thermostat set points are changed. For example, in figure 4.15, Case 5 has 
highest energy consumption in the month of January, while case 3 has highest energy consumption in months of May and October. The energy consumption mainly depends on what the thermostat level is rather than the number of changes to the thermostat. It can also be observed that case 3 has electricity consumption in September while the other cases do not have any consumption. This is because case 4 and case 5 are at level 1 , but in case 3 , as the levels are updated daily, there is a possibility that the thermostat may be at level 2 in some of the days, thus justifying the energy consumption in September.

\section{Building Envelope}

To study the effect of building envelope on energy consumption a graph is plotted as shown in figure 4.17. The outputs are from three different simulation runs. In first run (case 1) the building envelope is at level 1. In second run (case 2), the building envelope is set at level 2. The model also has the capability to update the building envelope factor if there are any plans to upgrade building envelope. In the third simulation run (case 3) the building envelope is assumed to be upgraded in July. Thus the building envelope is at level 2 until July and is at level 1 for the remaining year. 


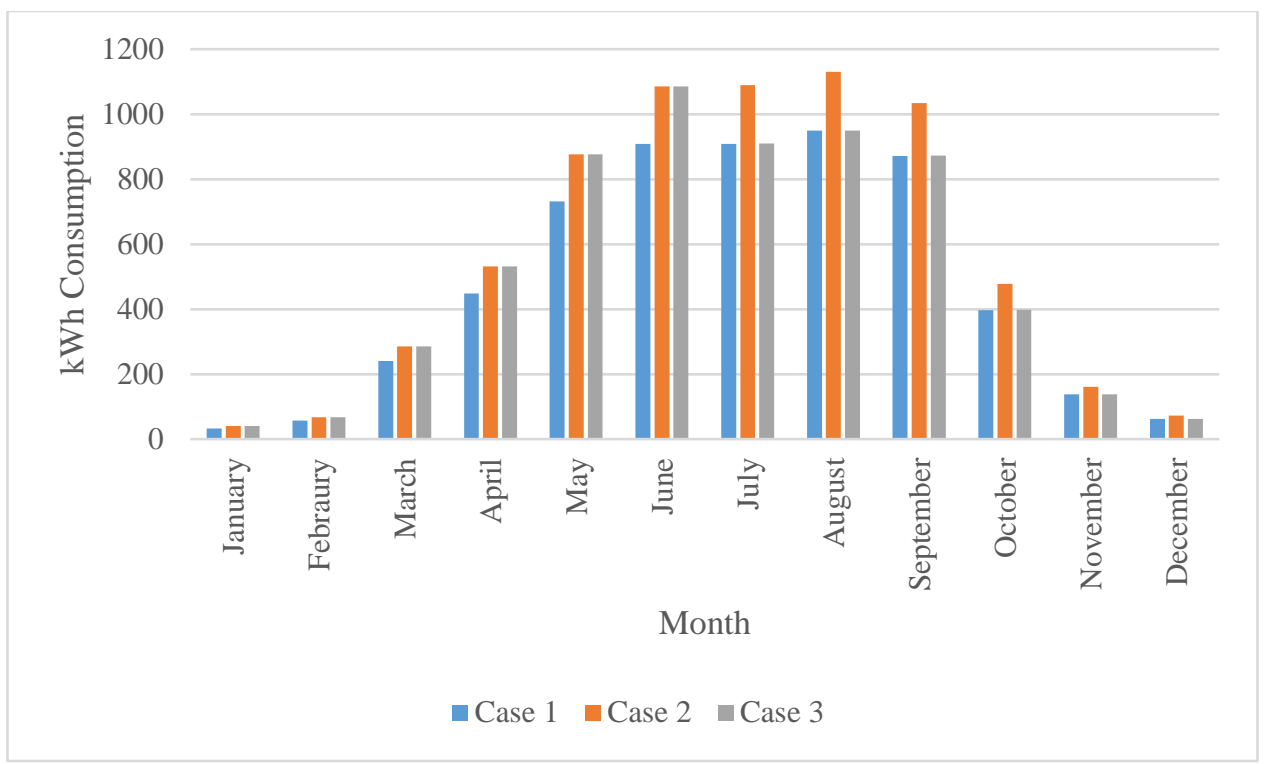

Figure 4.17: Electric Consumption vs Building Envelope Levels

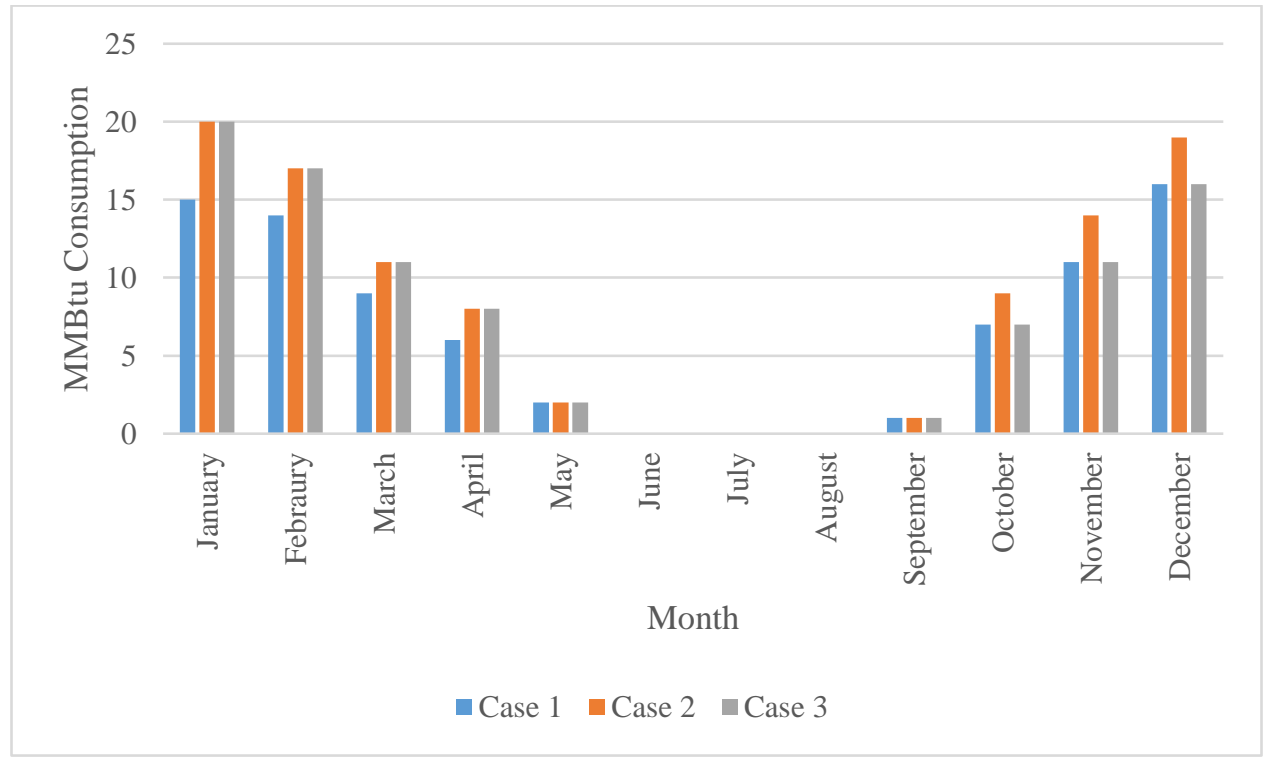

Figure 4.18: NG Consumption vs. Building Envelope Levels

The HVAC energy consumption depends on the building envelope, which is shown in the figure. It can be observed that the energy consumed in the case 2, is same as the energy consumed in case 3 for the first six months and case 1 in the last six months, as the building envelope is at level 2 till June $30^{\text {th }}$ and at level 1 for rest of the year. 


\section{Location of the User}

To understand the importance of the user's location while calculating the energy consumption, eight simulation runs, one run for each location are performed. Figure 4.19 gives us information regarding energy costs associated with a residential house with a similar configuration and thermal comfort levels in different census regions throughout the United States. It can be observed that the costs associated with heating (natural gas) is higher compared to costs associated with cooling (Electricity) in all the regions except for South Atlantic and West South Central.

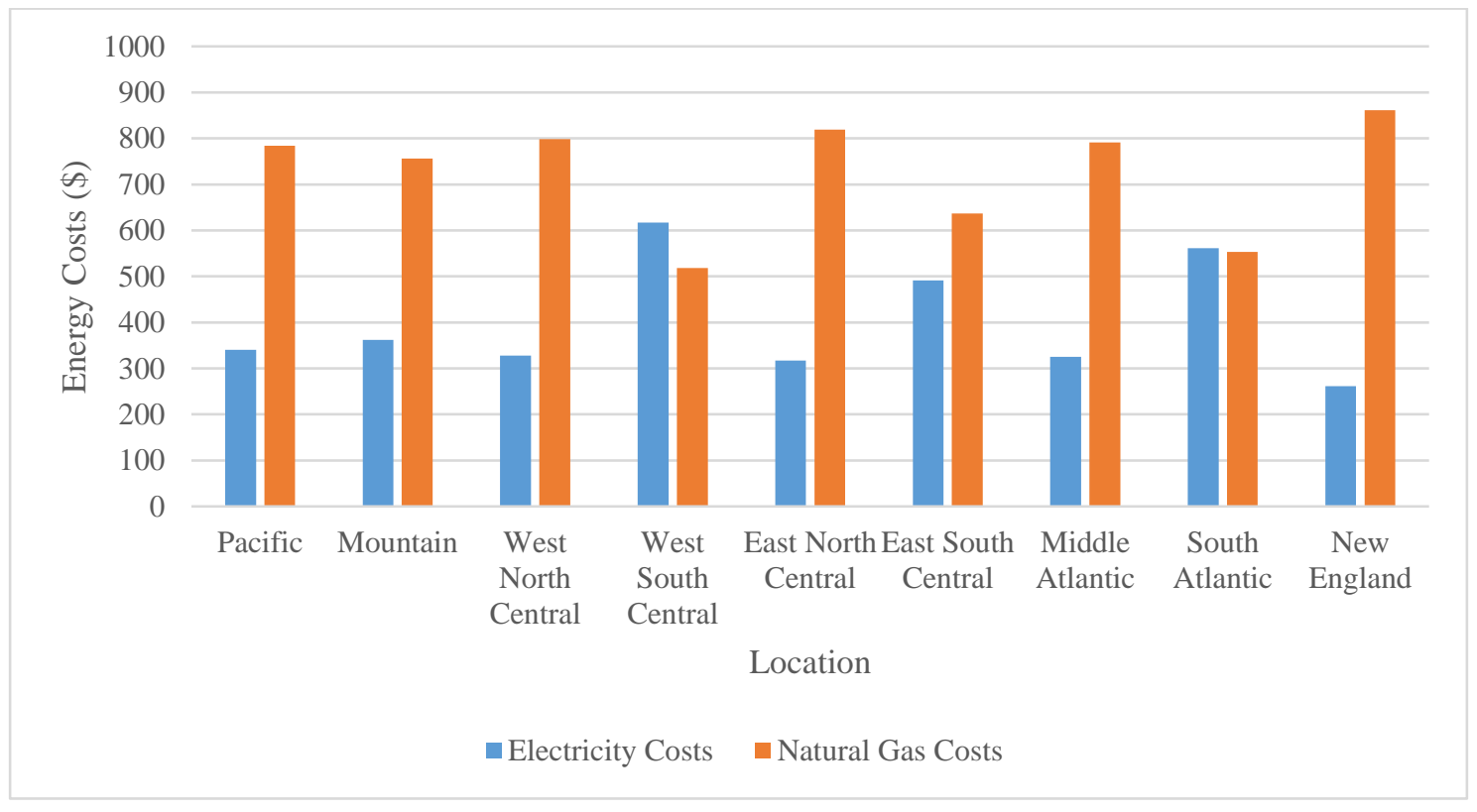

Figure 4.19: Total Energy Costs vs. Location of User

\subsection{Validation}

The model has been applied to simulate the HVAC system for a typical household located in the area of Morgantown, WV (i.e., South Atlantic region). The user has a heater with capacity of 85,000 Btu/hr. The user's house has five bed rooms, living room and a kitchen. However, there is only one regular thermostat in the living area. The occupancy of the house is very minimal, and the user prefers energy efficient thermostat set points (i.e., 60 
degree Fahrenheit in winter and 80 degree Fahrenheit in summer). The building envelope is tight with good insulation and very minimal insulation. The natural gas bills are obtained from the user and heater energy consumption is estimated after considering the energy consumption for cooking and the water heater. Heater energy consumption for the user turned out to be 32 MMBtu. The simulation is performed considering three bed rooms in order to mimic the configuration, as the user's house is not zoned and has only one regular thermostat. The occupancy pattern in the reference sheet is changed to meet the user's occupancy pattern. The model is run to simulate the energy consumption of the heater. The total energy consumed by the heater turned out to be $37 \mathrm{MMBtu}$. The difference between the simulated and the actual energy consumed is 5 MMBtu with an error of $15 \%$.

\subsection{Conclusion}

This chapter guides the user on how to use and input data into the model. The model also explains the user, what errors one might expect and how to resolve those errors. A sensitivity analysis is performed to know the effect of each factor on the energy consumption of the HVAC system with smart thermostat based BEMS. Finally a validation of the model is done to estimate the accuracy of the model. 


\section{Chapter 5 : Conclusions and Future Work}

\subsection{Conclusions}

A Windows-based simulation tool to estimate the energy consumption of a residential HVAC system with smart thermostat BEMS was developed. The tool was implemented in Microsoft Excel with Visual Basic. The technical features of the model include:

- Compatibility with Windows operating system

- Capability of handling up to five bedrooms with a living room and a kitchen

- Provides the user an option to select from nine different locations where his residential area is located.

The results of the model include:

- Total Monthly and Annual Energy Consumption

- Total Monthly and Annual Energy Costs Associated

\subsection{Future Work}

The model is developed based on Heuristic approach. Thus, the model can be a starting point for the future research studies. As the model uses heuristics, the model's results are good for an initial estimate but may not be accurate. The future studies should consider degree heating and cooling days instead of the season level. Calculating the thermal heating and cooling loads in each room can give the better results. Another critical factor is to consider the amount and type of insulation in the building. According to the literature thickness of the insulation should be optimal. In many cases, the building with heavy insulation will consume more energy. Thus, further studies should consider insulation type 
and thickness of the insulation in their calculations. It is also recommended that the future researchers should consider the effects of ventilation and exhaust air.

The model only has the capacity to consider two energy sources - Natural Gas and Electricity. However, these can be expanded to more energy sources like diesel, propane, etc. The scope of this study was only restricted to residential buildings; thus, no demand charge was considered. However, if the future studies want to focus on commercial buildings too, then they should account for Peak Demand. 


\section{Chapter 6 : References}

[1] W. C. Capehart, Barney L.; Kennedy, William J.; Turner, Guide to Energy Management (8th Edition) - International Version - Knovel. 2016.

[2] P. Beiter and T. Tian, 2015 Renewable Energy Data Book. Advanced Manufacturing Office, US Department of Energy, 2015.

[3] Y. Al Horr, M. Arif, M. Katafygiotou, A. Mazroei, A. Kaushik, and E. Elsarrag, "Impact of Indoor Environmental Quality on Occupant Well-Being and Comfort: A Review of The Literature," International Journal of Sustainable Built Environment, vol. 5, no. 1, pp. 1-11, Jun. 2016.

[4] M. Jin Woo and K. Jong-Jin, “ANN-Based Thermal Control Models for Residential Buildings," Building and Environment, vol. 45, no. 7, pp. 1612-1625, Jul. 2010.

[5] C. B. C. Lawrence, M. Woodward, "Smart Controls Usage in USA." Information Publication, U.S. Energy Information Administration, 2017.

[6] "Summary of Research Findings From the Programmable Thermostat Market." Information Publication, Energy Star, 2005.

[7] A. Ghaffarianhoseini, N. D. Dahlan, U. Berardi, A. Ghaffarianhoseini, N. Makaremi, and M. Ghaffarianhoseini, "Sustainable Energy Performances of Green Buildings: A Review of Current Theories, Implementations and Challenges," Renewable and Sustainable Energy Reviews, vol. 25, pp. 1-17, 2013.

[8] T. Markis and J. A. Paravantis, "Energy Conservation in Small Enterprises," Energy and Buildings, vol. 39, no. 4, pp. 404-415, Apr. 2007. 
[9] J. R. McKay, "The Effect of Wall and Roof Insulation on Energy Consumption for Environmental Control in a Small, 225-Square Foot Outside Plant Building,” INTELEC '87 - The Ninth International Telecommunications Energy Conference, Stockholm, Sweden, 1987, pp. 543-550.

[10] S. N. J. Al-Saadi and K. S. Al-Jabri, "Energy-Efficient Envelope Design for Residential Buildings: A Case Study in Oman," 2017 Smart City Symposium Prague (SCSP), Prague, Czech Republic, 2017, pp. 1-8.

[11] R. V. Conte, "Reducing Annual Energy Consumption by Proper Selection of HVAC Equipment," IECEC 96. Proceedings of the 31st Intersociety Energy Conversion Engineering Conference, Washington, DC, USA, 1996, vol. 2, pp. 1428-1433.

[12] V. S. Anantapantula, "Air-Conditioning System Performance with Heat Reclaim and Economizer Operation,” University of Missouri - Rolla, 1993.

[13] J. Jones, M. Navvab, and Y. Hill, "Operation and Climate Impact on Electrical Demand for Institutional Buildings," Conference Record of the 1992 IEEE Industry Applications Society Annual Meeting, Houston, TX, 1992, pp. 1852-1857.

[14] J. Ingersoll and J. Huang, "Heating Energy Use Management in Residential Buildings by Temperature Control," Energy and Buildings, vol. 8, no. 1, pp. 27-35, Feb. 1985.

[15] W. Guo and D. W. Nutter, "Setback and Setup Temperature Analysis for a Classic Double-Corridor Classroom Building," Energy and Buildings, vol. 42, no. 2, pp. 189-197, Feb. 2010. 
[16] T. Malinick, N. Wilairat, J. Holmes, and L. Perry, "Destined to Disappoint: Programmable Thermostat Savings are Only as Good as the Assumptions about Their Operating Characteristics," ACEEE Summer Study on Energy Efficiency in Buildings, Pacific Grove, CA, 2012.

[17] D. Cross and D. Judd, "Automatic Setback Thermostats: Measure Persistence and Customer Behavior | Energy Efficiency Program Library," Proceedings of the International Energy Program Evaluation Conference, Chicago, IL, 1997.

[18] J. R. Smith et al., "RFID-Based Techniques for Human-Activity Detection," Communications of the ACM, New York, NY, vol. 48, no. 9, p. 39, Sep. 2005.

[19] G. Gao and K. Whitehouse, "The Self-Programming Thermostat: Optimizing Setback Schedules Based on Home Occupancy Patterns," Proceedings of the First ACM Workshop on Embedded Sensing Systems for Energy-Efficiency in Buildings BuildSys '09, New York, NY, 2009, p. 67.

[20] M. Gupta, S. S. Intille, and K. Larson, "Adding GPS-Control to Traditional Thermostats: An Exploration of Potential Energy Savings and Design Challenges," Springer, Berlin, Heidelberg, 2009, pp. 95-114.

[21] B. Qela and H. T. Mouftah, "Observe, Learn, and Adapt (OLA)—An Algorithm for Energy Management in Smart Homes Using Wireless Sensors and Artificial Intelligence," IEEE Transactions on Smart Grid, vol. 3, no. 4, pp. 2262-2272, Dec. 2012.

[22] C. Aarish, M. Perussi, A. Rietz, and D. Korn, "Evaluation of the 2013-2014 Programmable and Smart Thermostat Program." Information Publication, The 
Cadmus Group, 2015.

[23] M. Muratori, V. Marano, R. Sioshansi, and G. Rizzoni, "Energy Consumption of Residential HVAC Systems: A Simple Physically-Based Model," 2012 IEEE Power and Energy Society General Meeting, San Diego, CA, 2012, pp. 1-8.

[24] J. A. White and R. Reichmuth, "Simplified Method for Predicting Building Energy Consumption Using Average Monthly Temperatures," IECEC 96. Proceedings of the 31 st Intersociety Energy Conversion Engineering Conference, Washington, DC, 1996, vol. 3, pp. 1834-1839.

[25] R. Zhou, Y. Pan, Z. Huang, and Q. Wang, "Building Energy Use Prediction Using Time Series Analysis," 2013 IEEE 6th International Conference on ServiceOriented Computing and Applications, Koloa, HI, 2013, pp. 309-313.

[26] T. Olofsson and S. Andersson, "Long-term energy demand predictions based on short-term measured data," Energy and Buildings, vol. 33, no. 2, pp. 85-91, Jan. 2001.

[27] “Monthly New Residential Construction, August 2017," Information Publication, U.S. Census Bureau, U.S. Department of Housing and Urban Development, 2017

[28] "Degree-Days - Energy Explained, Your Guide to Understanding Energy." Information Publication, U.S. Energy Information Administration, 2017.

[29] L. Biswas, "What Should Be Standard Size of Rooms in Residential Building? - A Civil Engineer." [Online]. Available: http://www.acivilengineer.com/2017/08/whatshould-bestandard-size-of-rooms- 
in.html. [Accessed: 24-May-2018].

[30] "What Temperature Should You Set Your Thermostat? (2017)." [Online]. Available: $\quad$ http://www.stansac.com/what-temperature-should-you-set-yourthermostat-in-summer-and-winter-with-statistics/. [Accessed: 19-Mar-2018]. 


\subsection{VBA Code}

\section{Chapter 7 : Appendix}

\subsubsection{Sheet (User Manual)}

Private Sub CommandButton1_Click()

Dim r As Double

Dim k, m, o, rooms, thermostat, BE As Integer

$\mathrm{m}=1$

$\mathrm{o}=1$

Dim bedrooms As Variant

Worksheets("Inputs").Cells(1000, 1000) = ""

Worksheets("Inputs").Cells(1001, 1000) = "'

ErrorHandler:

bedrooms = InputBox("No. of Bedrooms", "Bedrooms")

If IsNumeric(bedrooms) Then

If (bedrooms > 5) Then

MsgBox "Error- The simulation only has the capacity to handle 5 bedrooms"

GoTo ErrorHandler

ElseIf (bedrooms > 0 And bedrooms <6) Then

bedrooms $=$ bedrooms

ElseIf (bedrooms $=0$ ) Then

MsgBox "Error- Enter a number greater than 0"

GoTo ErrorHandler

End If

Else

MsgBox "Error- Enter a number"

GoTo ErrorHandler

End If 
Worksheets("Inputs").Activate

For $\mathrm{k}=1$ To 100

ActiveSheet.Range("A" \& k \& "").UnMerge

ActiveSheet.Range("B" \& k \& "").UnMerge

ActiveSheet.Range("C" \& k \& "").UnMerge

ActiveSheet.Range("D" \& k \& "").UnMerge

Next k

ActiveSheet.Range("A1:E200").Borders.LineStyle = xlContinuous

ActiveSheet.Range("A:E").Interior.ColorIndex = 2

ActiveSheet.Rows("1:1000"). RowHeight = 15

ActiveSheet.Range("A:E") = ""

ActiveSheet.Cells $(2,1)=$ "No. of Bedrooms"

ActiveSheet.Cells $(2,2)=$ bedrooms

ActiveSheet.Cells $(5,1)=$ "Room Name"

ActiveSheet.Cells $(5,4)=$ "Volume"

ActiveSheet.Cells (5, 3) = "Building Envelope"

ActiveSheet.Cells $(5,5)=$ "Thermostat"

rooms $=$ bedrooms

rooms $=$ rooms $+1+1$

For $\mathrm{k}=2$ To rooms +1

If $\mathrm{k}<$ rooms Then

ActiveSheet.Cells $(\mathrm{k}+4,1)=$ "bed room " \& o

$\mathrm{o}=\mathrm{o}+1$

ElseIf $\mathrm{k}=$ rooms Then

ActiveSheet.Cells $(\mathrm{k}+4,1)=$ "Living Room"

ElseIf $\mathrm{k}=$ rooms +1 Then 
ActiveSheet.Cells $(\mathrm{k}+4,1)=$ "Kitchen"

End If

ActiveSheet.Cells $(\mathrm{k}+4,1)$.Interior.ColorIndex $=28$

With ActiveSheet.Cells $(\mathrm{k}+4,3)$.Validation

.Delete

'replace "=A1:A6" with the range the data is in.

.Add Type:=xlValidateList, AlertStyle:=xlValidAlertStop,

Operator:=xlBetween, Formula1:="=Reference!H11:H12"

.IgnoreBlank $=$ True

.InCellDropdown $=$ True

.InputTitle $=" "$

ErrorTitle $=" "$

.InputMessage $=" "$

.ErrorMessage = " "

ShowInput $=$ True

.ShowError $=$ True

End With

With ActiveSheet.Cells $(\mathrm{k}+4,5)$.Validation

.Delete

'replace "=A1:A6" with the range the data is in.

.Add Type:=xlValidateList, AlertStyle:=xlValidAlertStop,

Operator:=xlBetween, Formula1:="=Reference!G11:G12"

.IgnoreBlank $=$ True

.InCellDropdown $=$ True

.InputTitle $=$ ""

.ErrorTitle $=$ ""

.InputMessage = ""

.ErrorMessage = "" 
ShowInput $=$ True

.ShowError $=$ True

End With

With ActiveSheet.Cells $(\mathrm{k}+4,4)$.Validation

.Delete

'replace "=A1:A6" with the range the data is in.

.Add Type $:=x l$ ValidateList, AlertStyle:=xlValidAlertStop, _

Operator:=xlBetween, Formula1:="=Reference!I11:I13"

.IgnoreBlank $=$ True

.InCellDropdown $=$ True

.InputTitle $=$ ""

ErrorTitle $=" "$

.InputMessage $=" "$

.ErrorMessage = " "

ShowInput $=$ True

ShowError $=$ True

End With

ActiveSheet.Cells $(\mathrm{k}+4$, 5).Interior.ColorIndex $=6$

ActiveSheet.Cells $(\mathrm{k}+4,3)$.Interior.ColorIndex $=36$

ActiveSheet.Cells $(\mathrm{k}+4,4)$.Interior.ColorIndex $=42$

Next k

MsgBox "Select a Level Building Envelope, Volume, Thermostat"

ActiveSheet.Columns("A").AutoFit

ActiveSheet.Columns("C:E").AutoFit

ActiveSheet.Cells $(\mathrm{k}+10,1)$.WrapText $=$ True

ErrorHandler7: 
$\mathrm{BE}=$ InputBox ("Will there be any improvements for the Building Envelope in Future" + vbCrLf + "1 - Yes" + vbCrLf + "2 - No", "Building Envelope Improvements")

If IsNumeric(BE) Then

If $(\mathrm{BE}>=3)$ Or $(\mathrm{BE}=0)$ Then

MsgBox "Error- Invalid Selection"

GoTo ErrorHandler7

ElseIf $(\mathrm{BE}>0$ And $\mathrm{BE}<3)$ Then

If $(\mathrm{BE}=1)$ Then

ActiveSheet.Cells $(\mathrm{k}+10,2)=$ "YES"

ErrorHandler8:

$\mathrm{BE}=$ InputBox $("$ When will the building envelope improvements take place" + vbCrLf + "1 - January" + vbCrLf + "2 - Febraury" + vbCrLf + "3 - March" + vbCrLf + "4

- April" + vbCrLf + "5 - May" + vbCrLf + "6 - June" + vbCrLf + "7 - July" + vbCrLf + "8 - August" + vbCrLf + "9 - September" + vbCrLf + "10 - October" + vbCrLf + "11 November" + vbCrLf + "12 - December", "Building Envelope Improvements")

If IsNumeric(BE) Then

If $(\mathrm{BE}>=13)$ Or $(\mathrm{BE}=0)$ Then

MsgBox "Error- Invalid Selection"

GoTo ErrorHandler8

ElseIf $(\mathrm{BE}>0$ And $\mathrm{BE}<13)$ Then

ActiveSheet.Range("A" \& k + 10 \& ":A" \& k + 15 \& "").Merge

ActiveSheet.Range("B" \& k + 10 \& ":B" \& k + 15 \& "").Merge

ActiveSheet.Cells $(\mathrm{k}+10,1)=$ "Will there be any improvements for the Building Envelope in Future, if any When?"

ActiveSheet.Cells $(\mathrm{k}+10,3)$.Formula $=$ "=VLOOKUP(" \& BE \& ",Reference!H26:K38,2,FALSE)"

ActiveSheet.Cells $(\mathrm{k}+10,4) \cdot$ Formula $=$ "=VLOOKUP $("$ \& BE \& ",Reference!H26:K38,4,FALSE)"

ActiveSheet.Cells $(1000,1000) \cdot$ Formula $=$ "=VLOOKUP(" \& BE \& ",Reference!H26:K38,4,FALSE)"

ActiveSheet.Range("C" \& k + $10 \&$ ":C" \& k + 15 \& "").Merge 
ActiveSheet.Range("D" \& k + 10 \& ":D" \& k + 15 \& "").Merge

End If

Else

MsgBox "Error- Enter a number"

GoTo ErrorHandler8

End If

Else

ActiveSheet.Range("A" \& k + 10 \& ":A" \& k + 14 \& "").Merge

ActiveSheet.Range("B" \& k + 10 \& ":B" \& k + 14 \& "").Merge

ActiveSheet.Cells $(\mathrm{k}+10,1)=$ "Will there be any improvements for the Building Envelope in Future?"

ActiveSheet.Cells $(\mathrm{k}+10,2)=$ "NO"

End If

End If

Else

MsgBox "Error- Enter a number"

GoTo ErrorHandler8

End If

ErrorHandler9:

thermostat = InputBox ("Do you change the Thermostat settings once calibrated" + vbCrLf + "1 - Yes" + vbCrLf + "2 - No", "Thermostat Changes per Year")

If IsNumeric(thermostat) Then

ActiveSheet.Range("A" \& k + 16 \& ":A" \& k + 20 \& "").Merge

ActiveSheet.Range("B" \& k + 16 \& ":B" \& k + 20 \& "").Merge

ActiveSheet.Cells $(\mathrm{k}+16,1)=$ "How frequently do you change the thermostat setting (\# of times/yr)?"

If thermostat $=1$ Then

ErrorHandler10:

$\mathrm{BE}=$ InputBox ("How frequently do you change the thermostat setting (\# of times/yr)", "Thermostat Changes per Year") 
If IsNumeric(BE) Then

ActiveSheet.Cells $(\mathrm{k}+16,2)=\mathrm{BE}$

ActiveSheet.Cells $(1001,1000)=\mathrm{BE}$

Else

GoTo ErrorHandler10

End If

ElseIf thermostat $=0$ Then

ActiveSheet.Cells $(\mathrm{k}+16,2)=$ "No Changes"

ElseIf thermostat $=2$ Then

ActiveSheet.Cells $(\mathrm{k}+16,2)=$ "No Changes"

Else

GoTo ErrorHandler9

End If

Else

GoTo ErrorHandler9

End If

Dim outages As Variant

ErrorHandler11:

outages = InputBox("Do you expect any power outages?" + vbCrLf + "1-Yes" + vbCrLf + "2-No", "Power Outages")

If IsNumeric(outages) Then

ActiveSheet.Range("A" \& k + 21 \& ":A" \& k + 25 \& "").Merge

ActiveSheet.Range("B" \& k + $21 \&$ ":B" \& k + $25 \&$ "").Merge

ActiveSheet.Cells $(\mathrm{k}+21,1)=$ "Do you expect any power outages?"

If outages $=1$ Then

ActiveSheet.Cells $(\mathrm{k}+21,2)=$ "Yes"

ActiveSheet.Range("A" \& k + 26 \& ":A" \& k + 32 \& "").Merge

ActiveSheet.Range("B" \& k + 26 \& ":B" \& k + $32 \&$ "").Merge 
ActiveSheet.Cells $(\mathrm{k}+26,1)=$ "What is the maximum number of power outages do you expect in a year?"

ActiveSheet.Cells $(\mathrm{k}+26,2)$.Interior.ColorIndex $=42$

ActiveSheet.Range("A" \& k + 33 \& ":A" \& k + 36 \& "").Merge

ActiveSheet.Range("B" \& k + 33 \& ":B" \& k + 36 \& "").Merge

ActiveSheet.Range("C" \& k + 33 \& ":C" \& k + 36 \& "").Merge

ActiveSheet.Cells $(\mathrm{k}+33,1)=$ "How long will the power outage last on an average?"

ActiveSheet.Cells $(\mathrm{k}+33,2)$.Interior.ColorIndex $=6$

ActiveSheet.Cells $(\mathrm{k}+33,3)$.Interior.ColorIndex $=36$

ActiveSheet.Cells $(\mathrm{k}+26,2) \cdot$.Interior.ColorIndex $=6$

\author{
ActiveSheet.Cells $(\mathrm{k}+21,1)$. WrapText $=$ True \\ ActiveSheet.Cells $(\mathrm{k}+26,1)$.WrapText $=$ True \\ ActiveSheet.Cells $(\mathrm{k}+33,1) \cdot$ WrapText $=$ True \\ With ActiveSheet.Cells $(\mathrm{k}+33,3)$.Validation \\ .Delete \\ 'replace "=A1:A6" with the range the data is in. \\ .Add Type:=xlValidateList, AlertStyle:=xlValidAlertStop, _ \\ Operator:=xlBetween, Formula1:="=Reference!J11:J12" \\ .IgnoreBlank $=$ True \\ .InCellDropdown $=$ True \\ .InputTitle $=$ "" \\ ErrorTitle $=$ "" \\ .InputMessage $=" "$ \\ .ErrorMessage = "" \\ .ShowInput $=$ True \\ .ShowError $=$ True \\ End With
}


ElseIf outages $=2$ Then

ActiveSheet.Cells $(\mathrm{k}+21,2)=$ "No"

Else

GoTo ErrorHandler11

End If

Else

GoTo ErrorHandler11

End If

ActiveSheet.Columns("A:E").VerticalAlignment = xlVAlignCenter

ActiveSheet.Range("A:E").HorizontalAlignment = xlCenter

End Sub

Private Sub CommandButton2_Click()

Dim a, c, d, e, m, n As Integer

Dim j As Integer

$\mathrm{a}=1$

$\mathrm{c}=$ Worksheets("Inputs").Cells(2, 2)

$\mathrm{d}=\mathrm{c}+$ Worksheets("Inputs").Cells(3, 2)

$\mathrm{e}=\mathrm{d}+2$

$\mathrm{m}=1$

$\mathrm{n}=1$

Worksheets("Inputs").Activate

ActiveSheet.Range("A:E") = ""

ActiveSheet.Range("A:E").Interior.ColorIndex $=2$

Worksheets("Inputs").Cells(1000, 1000) = "" 


\author{
Worksheets("Inputs").Cells(1001, 1000) = "" \\ Worksheets("User Manual").Activate \\ For $\mathrm{f}=1$ To e \\ If ( $\mathrm{f}>=1$ And $\mathrm{f}<=\mathrm{c}$ ) Then \\ On Error Resume Next \\ Worksheets("bedroom" \& m).Delete \\ $\mathrm{m}=\mathrm{m}+1$ \\ ElseIf ( $\mathrm{f}>\mathrm{c}$ And $\mathrm{f}<=\mathrm{d}$ ) Then \\ On Error Resume Next \\ Worksheets("bathroom" \& n).Delete \\ $\mathrm{n}=\mathrm{n}+1$ \\ ElseIf $(\mathrm{f}<\mathrm{e})$ Then \\ On Error Resume Next \\ Worksheets("Living room").Delete \\ ElseIf $(f=e)$ Then \\ On Error Resume Next \\ Worksheets("Kitchen").Delete \\ End If \\ Next $\mathrm{f}$ \\ On Error Resume Next \\ Worksheets("Summary").Delete \\ On Error Resume Next \\ Worksheets("Load Factor").Delete \\ On Error Resume Next \\ Worksheets("Energy Consumption").Delete \\ End Sub
}




\subsubsection{Sheet (Inputs)}

Private Sub CommandButton1_Click()

Dim r As Double

Dim bedrooms As Variant

Dim i, j, k, n, l, m, o, a, b, c, e, BE, h, z, y, x, w, v, sleeptime, sleephour, rooms As Integer $\mathrm{m}=1$

$0=1$

rooms $=$ Worksheets("Inputs").Cells $(2,2)+2$

For $\mathrm{k}=2$ To rooms +1

$\mathrm{z}=" "$

$\mathrm{x}=" "$

ActiveWorkbook.Sheets.Add After:=Worksheets(Worksheets.Count)

ActiveWindow.FreezePanes $=$ False

ActiveSheet.Rows("2:2").Select

ActiveWindow.FreezePanes $=$ True

ActiveSheet.Cells $(1,1)=$ "Day"

ActiveSheet.Cells $(1,2)=$ "Hour"

ActiveSheet.Cells $(1,3)$ = "Occupancy Random"

ActiveSheet.Cells $(1,4)=$ "Occupancy Level"

ActiveSheet.Cells $(1,5)=$ "Season Random"

ActiveSheet.Cells $(1,6)=$ "Season Level"

ActiveSheet.Cells(2,6).Formula= "=IF(E2<VLOOKUP(A2,Reference!\$A \$12:\$E\$16,3,TRUE), 1,IF(E2<VLOOKUP(A2,Re ference!\$A \$12:\$E\$16,4,TRUE),2,3))"

ActiveSheet.Cells(2, 6).Interior.ColorIndex $=28$

ActiveSheet.Cells $(1,7)=$ "Thermostat Random"

ActiveSheet.Cells $(1,8)=$ "Thermostat Level"

ActiveSheet.Cells $(1,9)$ = "Building Envelope Random"

ActiveSheet.Cells $(1,10)=$ "Building Envelope Level"

ActiveSheet.Cells $(1,11)=$ "Volume Random" 
ActiveSheet.Cells $(1,12)=$ "Volume Level"

ActiveSheet.Columns("B:Z").AutoFit

ActiveSheet.Range("A:Z").HorizontalAlignment = xlCenter

If $(\mathrm{k}<$ rooms $)$ Then

Sheets(ActiveSheet.Name).Name = "bedroom" \& o

ActiveSheet.Range("A1:Z20000").Borders.LineStyle = xlContinuous

ActiveSheet.Cells(2, $\quad$ 4).Formula $\quad=\quad=\operatorname{IF}(\mathrm{OR}($ $\operatorname{MOD}(\mathrm{A} 2,7)=6, \operatorname{MOD}(\mathrm{A} 2,7)=0), \operatorname{IF}(\mathrm{C} 2<(\operatorname{VLOOKUP}(\mathrm{B} 2, \operatorname{Reference} \$$ A $\$ 4: \$ D \$ 7,4, \mathrm{TRUE})$

),1,0),IF(C2<(VLOOKUP(B2,Reference!\$A4:\$D \$7,3,TRUE)),1,0))"

ActiveSheet.Cells $(\mathrm{k}+1,6)$.Interior.ColorIndex $=36$

$\mathrm{o}=\mathrm{o}+1$

If Worksheets("Inputs").Cells $(\mathrm{k}+4,4)=$ "' Or Worksheets("Inputs").Cells $(\mathrm{k}+4,4)$ $>=4$ Or Worksheets("Inputs").Cells $(\mathrm{k}+4,4)=0$ Then

$r=\operatorname{Rnd}()$

ActiveSheet.Cells $(2,11)=\operatorname{Round}(\mathrm{r}, 2)$

If ActiveSheet.Cells $(2,11)<=0.33$ Then

ActiveSheet.Cells $(2,12)=1$

ActiveSheet.Cells $(2,12)$.Interior.ColorIndex $=34$

ElseIf ActiveSheet.Cells $(2,11)>0.33$ And ActiveSheet.Cells $(2,11)<0.66$

Then

ActiveSheet.Cells $(2,12)=2$

ActiveSheet.Cells $(2,12)$.Interior.ColorIndex $=34$

ElseIf ActiveSheet.Cells $(2,11)>0.66$ Then

ActiveSheet.Cells $(2,12)=3$

ActiveSheet.Cells $(2,12)$.Interior.ColorIndex $=34$

End If

ElseIf Worksheets("Inputs").Cells(k + 4, 4) > 0 And Sheets("Inputs").Cells(k + 4, 4) $<4$ Then

ActiveSheet.Cells $(2,12)=$ Worksheets("Inputs").Cells $(\mathrm{k}+4,4)$

ActiveSheet.Cells $(2,12)$.Interior.ColorIndex $=34$

End If 
ElseIf $(k=$ rooms $)$ Then

Sheets(ActiveSheet.Name).Name = "Living room"

ActiveSheet.Range("A1:Z20000").Borders.LineStyle = xlContinuous

ActiveSheet.Cells(2, $\quad$ 4).Formula $\quad=\quad=\operatorname{IF}(\mathrm{OR}($ $\operatorname{MOD}(\mathrm{A} 2,7)=6, \operatorname{MOD}(\mathrm{A} 2,7)=0), \mathrm{IF}(\mathrm{C} 2<\operatorname{VLOOKUP}(\mathrm{B} 2$, Reference! \$F $\$$ 4:\$I $\$ 7,4, \mathrm{TRUE}), 1$, 0),IF(C2<VLOOKUP(B2,Reference!\$F\$4:\$I7,3,TRUE), 1,0))"

ActiveSheet.Cells $(\mathrm{k}+1,6)$.Interior.ColorIndex $=36$

If Worksheets("Inputs").Cells( $(\mathrm{k}+4,4)=$ "' Or Worksheets("Inputs").Cells(k + 4, 4) $>=4$ Or Worksheets("Inputs").Cells $(\mathrm{k}+4,4)=0$ Then

$\mathrm{r}=\operatorname{Rnd}()$

ActiveSheet.Cells $(2,11)=\operatorname{Round}(\mathrm{r}, 2)$

If ActiveSheet.Cells $(2,11)<=0.2$ Then

ActiveSheet.Cells $(2,12)=1$

ActiveSheet.Cells $(2,12)$.Interior.ColorIndex $=34$

ElseIf ActiveSheet.Cells(2, 11) $>0.2$ And ActiveSheet.Cells $(2,11)<0.5$ Then ActiveSheet.Cells $(2,12)=2$

ActiveSheet.Cells(2, 12).Interior.ColorIndex $=34$

ElseIf ActiveSheet.Cells $(2,11)>0.5$ Then

ActiveSheet.Cells $(2,12)=3$

ActiveSheet.Cells(2, 12).Interior.ColorIndex $=34$

End If

ElseIf Worksheets("Inputs").Cells(k + 4, 4) > 0 And Sheets("Inputs").Cells(k + 4, 4) $<4$ Then

ActiveSheet.Cells $(2,12)=$ Worksheets("Inputs").Cells $(\mathrm{k}+4,4)$

ActiveSheet.Cells(2, 12).Interior.ColorIndex $=34$

End If

ElseIf $(k=$ rooms +1$)$ Then

Sheets(ActiveSheet.Name).Name = "Kitchen"

ActiveSheet.Range("A1:Z20000").Borders.LineStyle = xlContinuous

ActiveSheet.Cells(2, $\quad$ 4).Formula $\quad=\quad=\operatorname{IF}(\mathrm{OR}($ $\operatorname{MOD}(\mathrm{A} 2,7)=6, \operatorname{MOD}(\mathrm{A} 2,7)=0), \operatorname{IF}(\mathrm{C} 2<\operatorname{VLOOKUP}(\mathrm{B} 2$, Reference! $\$ \mathrm{~K} \$ 4: \$ N \$ 8,4, \mathrm{TRUE})$, $1,0), \operatorname{IF}(\mathrm{C} 2<\mathrm{VLOOKUP}(\mathrm{B} 2$, Reference! $\$ \mathrm{~K} \$ 4: \$ N \$ 8,3, \mathrm{TRUE}), 1,0)) "$ 
ActiveSheet.Cells $(\mathrm{k}+1,6) \cdot$.Interior.ColorIndex $=36$

If Worksheets("Inputs").Cells( $k+4,4)=$ "" Or Worksheets("Inputs").Cells $(\mathrm{k}+4$, 4) $>=4$ Or Worksheets("Inputs").Cells $(k+4,4)=0$ Then

$$
\mathrm{r}=\operatorname{Rnd}()
$$

ActiveSheet.Cells $(2,11)=\operatorname{Round}(\mathrm{r}, 2)$

If ActiveSheet.Cells $(2,11)<=0.5$ Then

ActiveSheet.Cells $(2,12)=1$

ActiveSheet.Cells $(2,12)$.Interior.ColorIndex $=34$

ElseIf ActiveSheet.Cells $(2,11)>0.5$ And ActiveSheet.Cells $(2,11)<0.75$

Then

ActiveSheet.Cells $(2,12)=2$

ActiveSheet.Cells $(2,12)$.Interior.ColorIndex $=34$

ElseIf ActiveSheet.Cells $(2,11)>0.75$ Then

ActiveSheet.Cells $(2,12)=3$

ActiveSheet.Cells(2, 12).Interior.ColorIndex $=34$

End If

ElseIf Worksheets("Inputs").Cells $(\mathrm{k}+4,4)>0$ And Sheets("Inputs").Cells(k + 4, 4) $<4$ Then

ActiveSheet.Cells $(2,12)=$ Worksheets("Inputs").Cells $(\mathrm{k}+4,4)$

ActiveSheet.Cells $(2,12)$.Interior.ColorIndex $=34$

End If

End If

If Worksheets("Inputs").Cells( $k+4,3)=$ "' Or Worksheets("Inputs").Cells(k + 4, 3) $>=3$ Or Worksheets("Inputs").Cells $(\mathrm{k}+4,3)=0$ Then

$$
\begin{aligned}
\mathrm{z} & =\operatorname{Rnd}() \\
\mathrm{z} & =\text { Application.WorksheetFunction.Round }(\mathrm{z}, 2) \\
\text { If } \mathrm{z} & <=0.5 \text { Then } \\
\mathrm{BE} & =1
\end{aligned}
$$

Elself $z>0.5$ Then

$$
\mathrm{BE}=2
$$

End If 
ElseIf Worksheets("Inputs").Cells(k + 4, 3) >0 And Worksheets("Inputs").Cells(k $+4,3)<3$ Then

$$
\mathrm{BE}=\text { Worksheets("Inputs").Cells }(\mathrm{k}+4,3)
$$

End If

ActiveSheet.Cells $(2,26)=\mathrm{k}$

If Worksheets("Inputs").Cells $(\mathrm{k}+4,5)=$ "" Or Worksheets("Inputs").Cells $(\mathrm{k}+4$, 5) $>=3$ Or Worksheets("Inputs").Cells $(\mathrm{k}+4,5)=0$ Then

$$
\begin{aligned}
& x=\operatorname{Rnd}() \\
& x=\operatorname{Round}(x, 2)
\end{aligned}
$$

ActiveSheet.Cells $(2,7)=\mathrm{x}$

ActiveSheet.Cells $(2,8)$.Interior.ColorIndex $=34$

ActiveSheet.Cells $(2$,

ElseIf Worksheets("Inputs").Cells(k + 4, 5) > 0 And Sheets("Inputs").Cells(k + 4, 5) $<3$ Then

ActiveSheet.Cells $(2,8)=$ Worksheets("Inputs").Cells $(\mathrm{k}+4,3)$

End If

$\mathrm{h}=$ Worksheets("Inputs").Cells(1000, 1000)

ActiveSheet.Cells $(2,26)=\mathrm{h}$

ActiveSheet.Range("B:B").NumberFormat = "h:mm AM/PM; @"

$\mathrm{y}=$ Worksheets("Inputs").Cells(1001, 1000)

If $\mathrm{y}=$ "" Then

$\mathrm{y}=1$

End If

$\mathrm{y}=365 / \mathrm{y}$

$\mathrm{w}=1$

For $\mathrm{i}=0$ To 364

If $\mathrm{w}=0$ Then

sleephour $\quad=\quad$ (Worksheets("Reference").Range("M20")

Worksheets("Reference").Range("L20"))

Worksheets("Reference").Range("L20")

Rnd()

sleephour $=$ Round $($ sleephour, 0$)$ 


\section{End If}

If $(\mathrm{i}=\mathrm{h})$ Then 'Building Envelope Change Assignment

$$
\mathrm{BE}=1
$$

End If

If $\mathrm{i}>0$ And ( $\mathrm{i}$ Mod $\mathrm{y}=0$ ) Then ' Thermostat Factor Change

$$
\begin{aligned}
& r=\operatorname{Rnd}() \\
& x=\operatorname{Round}(r, 2)
\end{aligned}
$$

End If

$$
\begin{aligned}
& \mathrm{j}=1 \\
& \text { For } \mathrm{j}=0 \text { To } 23 \\
& \begin{array}{rl}
\text { For } \mathrm{c} & =1 \text { To } 2 \\
\mathrm{n}=\mathrm{i} & * 48+\mathrm{j} * 2+\mathrm{c} \\
\text { If } \mathrm{j}= & \text { sleephour Then } \\
\text { If } \mathrm{c}=1 \text { Then } \\
\quad \text { sleeptime }=(10-6) * \text { Rnd }()+6 \\
\quad \text { sleeptime }=2 *(\text { Round(sleeptime, } 0)) \\
\quad \mathrm{v}=\text { Rnd }() \\
\quad \mathrm{v}=\text { Application. WorksheetFunction.Round(v, 2) } \\
\quad \text { ActiveSheet.Cells(n }+1,3)=\text { Application.WorksheetFunction.Round }(\mathrm{v}, 2) \\
\quad \mathrm{w}=1
\end{array}
\end{aligned}
$$

End If

\section{End If}

If (sleeptime $>=w$ ) Then

$$
\text { ActiveSheet.Cells }(n+1,3)=v
$$

$$
\mathrm{w}=\mathrm{w}+1
$$

ElseIf (sleeptime $=w)$ Then

$$
\mathrm{W}=0
$$

$$
\text { sleeptime }=0
$$

Else

$\mathrm{v}=\operatorname{Rnd}()$ 
ActiveSheet.Cells $(\mathrm{n}+1,3)=$ Application.WorksheetFunction.Round $(\mathrm{v}, 2)$

End If

ActiveSheet.Cells $(n+1,6) \cdot$ Interior.ColorIndex $=28$

ActiveSheet.Cells $(n+1,4)$.Interior.ColorIndex $=28$

ActiveSheet.Cells $(\mathrm{n}+1,2)=\operatorname{TimeSerial}(\mathrm{j}, 30 *(\mathrm{c}-1), 0)$

ActiveSheet.Cells $(n+1,1)=i+1$

ActiveSheet.Cells $(2,8)$.Interior.ColorIndex $=34$

ActiveSheet.Cells $(\mathrm{n}+1,7)=\mathrm{x}$

ActiveSheet.Cells $(n+1,9)=z$

ActiveSheet.Cells $(\mathrm{n}+1,10)=\mathrm{BE}$

ActiveSheet.Cells $(n+1,10)$.Interior.ColorIndex $=34$

If $(\mathrm{k}=2)$ Then

If $(\mathrm{n} \operatorname{Mod} 16=1)$ Then

$\mathrm{r}=\operatorname{Rnd}()$

$\mathrm{e}=$ Application. WorksheetFunction.Round(r, 2)

ActiveSheet.Cells $(\mathrm{n}+1,5)=\mathrm{e}$

ElseIf ( $\mathrm{n}$ Mod $16=0$ Or $\mathrm{n}$ Mod $16>1)$ Then

ActiveSheet.Cells $(\mathrm{n}+1,5)=\mathrm{e}$

End If

ElseIf $(k>2)$ Then

ActiveSheet.Cells $(\mathrm{n}+1,5)=$ Worksheets("bedroom1").Cells $(\mathrm{n}+1,5)$

End If

Next c

Next j

Next i

ActiveSheet.Columns("B").AutoFit

ActiveSheet.Cells $(2, \quad 8)$.Formula $=$ "=IF(G2="""",Inputs!\$E\$" \& $\mathrm{k}+4 \quad \&$ ",IF(G2<VLOOKUP(A2,Reference!\$G\$19:\$J22,3,TRUE),1,2))"

Next k 
Worksheets("Inputs").Activate

End Sub

Private Sub CommandButton2_Click()

Dim a, c, e, m, n As Integer

Dim j As Integer

$\mathrm{a}=1$

$\mathrm{c}=$ Worksheets("Inputs").Cells(2, 2) + 2

$\mathrm{m}=1$

ActiveWorkbook.Sheets.Add After:=Worksheets(Worksheets.Count)

Sheets(ActiveSheet.Name).Name = "Load Factor"

ActiveSheet.Cells $(2,1)=$ "Floor Area"

ActiveSheet.Range("A1:Z20000").Borders.LineStyle = xlContinuous

ActiveWindow.FreezePanes $=$ False

ActiveSheet.Rows("2:2").Select

ActiveWindow.FreezePanes $=$ True

Worksheets("Load Factor").Cells(5, c + 1) = "Average Load Factor"

Worksheets("Load Factor").Cells(5, 1) = "Day"

Worksheets("Load Factor").Cells(5, 2) = "Time"

Worksheets("Load Factor").Cells(6, 1).Formula = "=Kitchen!A2"

Worksheets("Load Factor").Cells(6, 2).Formula = "=Kitchen!B2"

Worksheets("Load Factor").Range("A6").Copy

Worksheets("Load Factor").Range("A7:A17526").Select

Worksheets("Load Factor").Paste

Worksheets("Load Factor").Range("B6").Copy

Worksheets("Load Factor").Range("B7:B17526").Select

Worksheets("Load Factor").Paste

For $\mathrm{f}=1$ To $\mathrm{c}$

If $(f<=c-2)$ Then

Worksheets("bedroom" \& m).Activate 
Worksheets("Load Factor").Cells $(1, \mathrm{f}+2)=$ "Bedroom" \& m

Worksheets("Load Factor").Cells $(5, \mathrm{f}+2)=$ "Load Factor"

Worksheets("Load Factor").Cells(2, f + 2).Formula = "=VLOOKUP('bedroom" \& m \& "'!L2,Reference!A19:E22,2,FALSE)"

$m=m+1$

ElseIf $(\mathrm{f}<\mathrm{c})$ Then

Worksheets("Living room").Activate

Worksheets("Load Factor").Cells $(1, \mathrm{f}+2)=$ "Living room"

Worksheets("Load Factor").Cells $(5, \mathrm{f}+2)=$ "Load Factor"

Worksheets("Load Factor").Cells(2, f + 2).Formula $=$ "=VLOOKUP('Living room'!L2,Reference!A19:E22,4,FALSE)"

ElseIf $(f=c)$ Then

Worksheets("Kitchen").Activate

Worksheets("Load Factor").Cells $(1, f+2)=$ "Kitchen"

Worksheets("Load Factor").Cells $(5, \mathrm{f}+2)$ = "Load Factor"

Worksheets("Load Factor").Cells(2, $\mathrm{f}+\mathrm{+}$ 2). Formula = "=VLOOKUP('Kitchen'!L2,Reference!A19:E22,5,FALSE)"

End If

Worksheets("Load Factor").Cells(1, c + 3) = "Average Load Factor"

ActiveSheet.Range("D2").Copy

ActiveSheet.Range("D3:D17521").Select

ActiveSheet.Paste

ActiveSheet.Range("F2").Copy

ActiveSheet.Range("F3:F17521").Select

ActiveSheet.Paste

ActiveSheet.Columns("B:Z").AutoFit

ActiveSheet.Range("H2").Copy

ActiveSheet.Range("H3:H17521").Select 
ActiveSheet.Paste

ActiveSheet.Columns("B:Z").AutoFit

ActiveSheet.Cells $(1,13)=$ "Load Factor"

For $\mathrm{a}=1$ To 17520

For $\mathrm{j}=2$ To 75

If (ActiveSheet.Cells $(\mathrm{a}+1,4)=$ Worksheets("Reference").Cells $(\mathrm{j}, 18))$ Then

If (ActiveSheet.Cells $(\mathrm{a}+1,6)=$ Worksheets("Reference").Cells $(j, 19))$ Then

If (ActiveSheet.Cells $(\mathrm{a}+1,8)=$ Worksheets("Reference").Cells $(\mathrm{j}, 20))$ Then

If (ActiveSheet.Cells $(\mathrm{a}+1,10)=$ Worksheets("Reference").Cells(j, 21))

Then

If (ActiveSheet.Cells(2, 12) = Worksheets("Reference").Cells(j, 22))

Then

ActiveSheet.Cells $(\mathrm{a}+1,13)=$ Worksheets("Reference").Cells $(\mathrm{j}, 23)$

Worksheets("Load Factor").Cells(a + 5, f + 2) =

Worksheets("Reference").Cells(j, 23)

ActiveSheet.Cells $(\mathrm{a}+1,13)$.Interior.ColorIndex $=6$

End If

End If

End If

End If

End If

Next j

Next a

Next $f$

Worksheets("Load Factor").Cells(1, 10000).Formula = "=VLOOKUP(" \& c + 2 \& ", Reference!\$AJ\$1:\$AK\$26,2,FALSE)"

Worksheets("Load Factor").Cells(1, 10001).Formula $=$ "=VLOOKUP(" \& c + $3 \&$ ",Reference!\$AJ\$1:\$AK\$26,2,FALSE)"

Worksheets("Load Factor").Cells(5, c + 3) = "Average Load Factor"

Worksheets("Load Factor").Cells(6, c + 3).Formula = "=Round(SUMPRODUCT(\$C \$2:\$"

\& Worksheets("Load Factor").Cells(1, 10000) \& "\$2,C6:" \& Worksheets("Load 
Factor").Cells(1, 10000) \& "6)/sum(\$C \$2:\$" \& Worksheets("Load Factor").Cells(1, 10000) \& "\$2),2)"

Worksheets("Load Factor").Activate

ActiveSheet.Range(Worksheets("Load Factor").Cells(1, 10001) \& "6").Copy

ActiveSheet.Range(Worksheets("Load Factor").Cells(1, 10001) \& "7:" \& Worksheets("Load Factor").Cells(1, 10001) \& "17530").Select

ActiveSheet.Paste

ActiveSheet.Columns("B:Z").AutoFit

ActiveSheet.Range("A:Z").HorizontalAlignment = xlCenter

Worksheets("Inputs").Activate

Worksheets("Load Factor").Range("B:B").NumberFormat = "h:mm AM/PM; @"

End Sub

Private Sub CommandButton3_Click()

Dim e, i, j, n As Integer

Dim x, y As Double

$\mathrm{e}=$ Worksheets("Inputs").Cells(2, 2)

$\mathrm{e}=\mathrm{e}+$ Worksheets("Inputs").Cells( 3,2$)$

$\mathrm{e}=\mathrm{e}+2$

Dim region As Variant

ErrorHandler7:

region = InputBox ("Where do you stay?" + vbCrLf + "1-Pacific " + vbCrLf + "2-Mountain " + vbCrLf + "3-West North Central" + vbCrLf + "4- West South Central" + vbCrLf + "5East North Central" + vbCrLf + "6-East South Central" + vbCrLf + "7-Middle Atlantic" + vbCrLf + "8-South Atlantic" + vbCrLf + "9-New England", "Location")

If IsNumeric(region) Then

If (region >= 10) Or (region =0) Then

MsgBox "Error- Invalid Selection"

GoTo ErrorHandler7 
ElseIf (region > 0 And region < 10) Then

End If

Else

MsgBox "Error- Enter a number"

GoTo ErrorHandler7

End If

Worksheets("Reference").Activate

For $\mathrm{i}=1$ To 12

ActiveSheet.Range("L" \& i + 26).Formula = "=HLOOKUP(" \& region \& ",\$B \$81:\$J94," $\& \mathrm{i}+2 \&$ ",FALSE)"

ActiveSheet.Range("M" \& i + 26).Formula = "=HLOOKUP(" \& region \& ",\$A \$96:\$J109," \& i + 2 \& ",FALSE)"

Next i

ActiveWorkbook.Sheets.Add After:=Worksheets(Worksheets.Count)

Sheets(ActiveSheet.Name).Name = "Energy Consumption"

ActiveSheet.Range("A1:Z20000").Borders.LineStyle = xlContinuous

Worksheets("Energy Consumption").Cells $(1,1)=$ "Date"

Worksheets("Energy Consumption").Cells $(1,2)=$ "Time"

Worksheets("Energy Consumption").Cells(1, 3) = "Average Load Factor"

Worksheets("Energy Consumption").Cells(2, 1).Formula = "=Kitchen!A2"

Worksheets("Energy Consumption").Cells(2, 2).Formula = "=Kitchen!B2"

Worksheets("Load Factor").Cells(1, 10000).Formula $=$ "=VLOOKUP(" \& e + $3 \&$ ",Reference!\$AJ\$1:\$AK\$26,2,FALSE)"

Worksheets("Energy Consumption").Cells(2, 3).Formula = "='Load Factor'!" \& Worksheets("Load Factor").Cells(1, 10000) \& "6"

Worksheets("Energy Consumption").Activate

ActiveWindow.FreezePanes $=$ False

ActiveSheet.Rows("2:2").Select

ActiveWindow.FreezePanes $=$ True

ActiveSheet.Cells(2, 1).Copy 
ActiveSheet.Range("A3:A17521").Select

ActiveSheet.Paste

ActiveSheet.Cells(2, 2).Copy

ActiveSheet.Range("B3:B17521").Select

ActiveSheet.Paste

ActiveSheet.Cells(2, 3).Copy

ActiveSheet.Range("C3:C17521").Select

ActiveSheet.Paste

Dim Fuel, COP, MMBtu, tonnage, CMMBtu, CkW, heaterkw As Variant

ActiveSheet.Cells $(1,5)=$ "Energy Costs"

ErrorHandler:

Fuel = InputBox ("How do you heat your home" + vbCrLf + "1-NG " + vbCrLf + "2Electricity ", "Type of Fuel")

If IsNumeric(Fuel) Then

If (Fuel >= 3) Or (Fuel = 0) Then

MsgBox "Error- Invalid Selection"

GoTo ErrorHandler

Elself (Fuel > 0 And Fuel < 3) Then

End If

Else

MsgBox "Error- Enter a number"

GoTo ErrorHandler

End If

If Fuel $=1$ Then

ActiveSheet.Cells $(1,4)=$ "Electricity Consumption"

ActiveSheet.Cells $(1,6)=$ "Electricity Costs"

ActiveSheet.Cells $(1,5)=$ "NG Consumption"

ActiveSheet.Cells $(1,7)=$ "NG Costs"

ErrorHandler1: 
MMBtu = InputBox("Capacity of heater in MMBtu/hr", "MMBtu/hr")

If IsNumeric(MMBtu) Then

If $($ MMBtu $=0)$ Then

MsgBox "Error- Invalid Entry"

GoTo ErrorHandler1

End If

Else

MsgBox "Error- Enter a number"

GoTo ErrorHandler1

End If

ErrorHandler2:

CMMBtu = InputBox ("Cost of NG in MMBtu", "\$/MMBtu")

If IsNumeric(CMMBtu) Then

If $(\mathrm{CMMBtu}=0)$ Then

MsgBox "Error- Invalid Entry"

GoTo ErrorHandler2

End If

Else

MsgBox "Error- Enter a number"

GoTo ErrorHandler2

End If

Worksheets("Reference").Range("M12") = MMBtu

Worksheets("Reference").Range("N12") = CMMBtu

Worksheets("Reference").Range("M14") = MMBtu

Worksheets("Reference").Range("N14") = CMMBtu

ActiveSheet.Range("H1") = "Total kWh Energy Consumption"

ActiveSheet.Range("H3") = "Total kWh Costs"

ActiveSheet.Range("I1") = "Total MMBtu Energy Consumption"

ActiveSheet.Range("I3") = "Total MMBtu Costs" 
ActiveSheet.Range("G2").Formula = "=E2*" \& CMMBtu

ActiveSheet.Cells(2, 7).Copy

ActiveSheet.Range("G3:G17521").Select

ActiveSheet.Paste

ActiveSheet.Range("H2").Formula = "=ROUNDUP(SUM(D2:D17521),0)"

ActiveSheet.Range("I2").Formula = "=ROUNDUP(SUM(E2:E17521),0)"

ActiveSheet.Range("H4").Formula = "=ROUNDUP(SUM(F2:F17521),0)"

ActiveSheet.Range("I4").Formula = "=ROUNDUP(SUM(G2:G17521),0)"

ActiveSheet.Range("H1:I1, H3:I3").Interior.ColorIndex = 6

ActiveSheet.Range("H1:I1, H3:I3").Interior.ColorIndex = 8

Else

ActiveSheet.Cells $(1,4)=$ "AC kWh Consumption"

ActiveSheet.Cells $(1,6)=$ "AC Energy Costs"

ActiveSheet.Cells $(1,5)$ = "Heater kWh Consumption"

ActiveSheet.Cells $(1,7)=$ "Heater Energy Costs"

ErrorHandler10:

heaterkw = InputBox("Capacity of heater in kW", "kW")

If IsNumeric(heaterkw) Then

If (heaterkw $=0)$ Then

MsgBox "Error- Invalid Entry"

GoTo ErrorHandler10

End If

Else

MsgBox "Error- Enter a number"

GoTo ErrorHandler10

End If

Worksheets("Reference").Range("M12") = heaterkw

Worksheets("Reference").Range("M14") = heaterkw

ActiveSheet.Range("H1") = "Total AC Energy Consumption"

ActiveSheet.Range("H3") = "Total AC Energy Costs" 
ActiveSheet.Range("I1") = "Total Heater Energy Consumption"

ActiveSheet.Range("I3") = "Total Heater Energy Costs"

"'=ROUNDUP(SUM(H2:H17521),0)"

ActiveSheet.Range("H2").Formula = "=ROUNDUP(SUM(D2:D17521),0)"

ActiveSheet.Range("I2").Formula = "=ROUNDUP(SUM(E2:E17521),0)"

ActiveSheet.Range("H4").Formula = "=ROUNDUP(SUM(F2:F17521),0)"

ActiveSheet.Range("I4").Formula = "=ROUNDUP(SUM(G2:G17521),0)"

ActiveSheet.Range("H1:I1, H3:I3").Interior.ColorIndex $=6$

ActiveSheet.Range("H1:I1, H3:I3").Interior.ColorIndex = 8

End If

\section{ErrorHandler3:}

tonnage $=$ InputBox $($ "Capacity of Air Conditioner in tonnage", "Tonnage")

If IsNumeric(tonnage) Then

If (tonnage $=0$ ) Then

MsgBox "Error- Invalid Entry"

GoTo ErrorHandler3

End If

Else

MsgBox "Error- Enter a number"

GoTo ErrorHandler3

End If

ErrorHandler8:

$\mathrm{COP}=$ InputBox ("COP of Air Conditioner", "COP")

If IsNumeric(COP) Then

If $(\mathrm{COP}=0)$ Then

MsgBox "Error- Invalid Entry" 


\section{GoTo ErrorHandler8}

End If

Else

MsgBox "Error- Enter a number"

GoTo ErrorHandler8

End If

\section{ErrorHandler4:}

$\mathrm{CkW}=\operatorname{InputBox}("$ Cost of Electricity in kWh", "\$/kWh")

If IsNumeric(CkW) Then

If $(\mathrm{CkW}=0)$ Then

MsgBox "Error- Invalid Entry"

GoTo ErrorHandler4

End If

Else

MsgBox "Error- Enter a number"

GoTo ErrorHandler4

End If

If $($ Fuel = 2) Then

ActiveSheet.Range("G2").Formula = "=E2*" \& CkW

ActiveSheet.Cells(2, 7).Copy

ActiveSheet.Range("G3:G17521").Select

ActiveSheet.Paste

End If

$\mathrm{kW}=$ tonnage $* 12 /(\mathrm{COP} * 3.412)$

Worksheets("Reference").Range("M13") = kW

Worksheets("Reference").Range("N13") = CkW

Worksheets("Reference").Cells(12, 15).Formula = "=round(VLOOKUP(" \& region \& ",Reference!\$A $\$ 26: \$ F \$ 35,5, F A L S E), 2) "$ 
Worksheets("Reference").Cells(13, 15).Formula = "=round(VLOOKUP(" \& region \& ",Reference!\$A26:\$F\$35,6,FALSE),2)"

Worksheets("Reference").Cells(14, 15).Formula = "=round(VLOOKUP(" \& region \& ",Reference!\$A26:\$F\$35,5,FALSE),2)"

Dim c, a, b, d As Integer

$\mathrm{c}=$ Worksheets("Inputs").Cells $(2,2)+4$

If Worksheets("Inputs").Cells(c + 21, 2) = "Yes" Then

Dim outages, outagetime As Integer

outages $=$ Worksheets $(" I n p u t s ") \cdot$ Cells $(c+26,2)$

outages $=($ outages -0$) * \operatorname{Rnd}()+0$

outages $=$ WorksheetFunction $\cdot$ Round $($ outages, 0$)$

If Worksheets("Inputs").Cells $(c+33,3)=$ "Days" Then

$\mathrm{a}=$ Worksheets("Inputs").Cells $(\mathrm{c}+33,2)$

$\mathrm{a}=\mathrm{a} / 365$

$\mathrm{b}=$ Worksheets("Inputs").Cells $(\mathrm{c}+33,2) * 48$

ElseIf Worksheets("Inputs").Cells $(c+33,3)=$ "Hours" Then

$\mathrm{a}=$ Worksheets("Inputs").Cells $(\mathrm{c}+33,2)$

$\mathrm{a}=\mathrm{a} / 8760$

$\mathrm{b}=$ Worksheets("Inputs").Cells $(\mathrm{c}+33,2) * 2$

End If

Else

outages $=0$

End If

$\mathrm{c}=0$

For $\mathrm{i}=0$ To 364

For $\mathrm{j}=1$ To 48

If $\operatorname{Rnd}()<=a$ Then

$\mathrm{c}=\mathrm{c}+1$

If $\mathrm{c}<=$ outages Then 
For $\mathrm{d}=1$ To $\mathrm{b}$

$\mathrm{n}=\mathrm{i} * 48+\mathrm{j}$

ActiveSheet.Cells $(\mathrm{n}+1,4)=0$

ActiveSheet.Cells $(n+1,5)=0$

If $\mathrm{j}>48$ Then

$\mathrm{j}=1$

$\mathrm{i}=\mathrm{i}+1$

End If

$\mathrm{j}=\mathrm{j}+1$

Next d

End If

$\mathrm{x}=\operatorname{Rnd}()$

End If

$$
\mathrm{n}=\mathrm{i} * 48+\mathrm{j}
$$

ActiveSheet.Cells(n $+1,1000)$.Formula $="=\operatorname{VLOOKUP}\left(\mathrm{A}^{\prime} \& \mathrm{n}+1 \&\right.$ ",Reference!\$K\$26:\$N\$38,4,TRUE)"

If ( $\mathrm{j}$ Mod $16=1)$ Then

$$
\mathrm{x}=\operatorname{Rnd}()
$$

End If

If $\mathrm{x}<$ ActiveSheet.Cells $(\mathrm{n}+1,1000)$ Then

ActiveSheet.Cells $\left(\mathrm{n}+1\right.$, 4).Formula $="=\operatorname{round}\left(\mathrm{C}^{\prime} \& \mathrm{n}+1 \&\right.$ "*0.5*Reference!M13,2)"

Else

ActiveSheet.Cells( $\mathrm{n}+1,5)$.Formula $="=\operatorname{round}(\mathrm{C} " \& \mathrm{n}+1 \&$ "*0.5*Reference!M12,2)"

\section{End If}

Next j

Next i

ActiveSheet.Range("ALL1:ALL19000") = ""

ActiveSheet.Range("F2").Formula = "=D2*" \& CkW

ActiveSheet.Cells(2, 6).Copy 
ActiveSheet.Range("F3:F17521").Select

ActiveSheet.Paste

ActiveSheet.Range("B:B").NumberFormat = "h:mm AM/PM; @"

ActiveSheet.Columns("A:Z").AutoFit

ActiveSheet.Range("F2").NumberFormat = Format(ActiveSheet.Range("F2"), "\#,\#\#0")

ActiveSheet.Range("A:Z").HorizontalAlignment = xlCenter

ActiveWorkbook.Sheets.Add After:=Worksheets(Worksheets.Count)

Sheets(ActiveSheet.Name).Name = "Summary"

Worksheets("Summary").Activate

$\mathrm{j}=1$

ActiveSheet.Cells $(1,1)=$ "Month"

ActiveSheet.Cells $(1,2)$ = "Electricity Consumption"

If Fuel $=1$ Then

ActiveSheet.Cells $(1,3)=$ "Natural Gas Consumption"

ActiveSheet.Cells $(1,4)=$ "Electric Costs"

ActiveSheet.Cells $(1,5)=$ "Natural Gas Costs"

Else

ActiveSheet.Cells $(1,3)=$ "Electric Costs"

End If

ActiveSheet.Cells $(2,1) \cdot$ Formula = "=Reference!I27"

ActiveSheet.Cells(2, 1).Copy

ActiveSheet.Range("A3:A13").Select

ActiveSheet.Paste

ActiveSheet.Cells(2, 1000).Formula $\quad=\quad$ =VLOOKUP('Energy

Consumption'!A2,Reference!\$A40:\$B \$51,1,TRUE)"

ActiveSheet.Cells(2, 1000).Copy

ActiveSheet.Range("ALL3:ALL17770").Select 
ActiveSheet.Paste

$\mathrm{kW}=0$

MMBtu $=0$

For $\mathrm{i}=1$ To 17520

If $($ ActiveSheet.Cells $(i, 1000)=$ ActiveSheet.Cells $(i+1,1000))$ And $i<17520$ Then

$$
\begin{aligned}
& \mathrm{kW}=\mathrm{kW}+\text { Worksheets("Energy Consumption").Cells( }(\mathrm{i}, 1,4) \\
& \mathrm{MMBtu}=\text { MMBtu + Worksheets("Energy Consumption").Cells }(\mathrm{i}+1,5)
\end{aligned}
$$

Else

If Fuel $=1$ Then

ActiveSheet.Cells $(\mathrm{j}+1,2)=\operatorname{Round}(\mathrm{kW}, 0)$

ActiveSheet.Cells $(j+1,3)=\operatorname{Round}($ MMBtu, 0$)$

Else

ActiveSheet.Cells $(\mathrm{j}+1,2)=\operatorname{Round}((\mathrm{kW}+\mathrm{MMBtu}), 0)$

End If

$$
\mathrm{j}=\mathrm{j}+1
$$

$\mathrm{kW}=0$

$$
\text { MMBtu }=0
$$

End If

Next i

ActiveSheet.Range("A:Z").HorizontalAlignment = xlCenter

If Fuel $=1$ Then

ActiveSheet.Cells(2, 4).Formula = "=roundup(B2*" \& CkW \& ",0)"

ActiveSheet.Cells(2, 4).Copy

ActiveSheet.Range("D3:D13").Select

ActiveSheet.Paste

ActiveSheet.Cells(2, 5).Formula = "=roundup $(\mathrm{C} 2 * " \&$ CMMBtu \& ",0)"

ActiveSheet.Cells(2, 5).Copy

ActiveSheet.Range("E3:E13").Select 
ActiveSheet.Paste

Else

ActiveSheet.Cells(2, 3).Formula = "=roundup(B2*" \& CkW \& ",0)"

ActiveSheet.Cells(2, 3).Copy

ActiveSheet.Range("C3:C13").Select

ActiveSheet.Paste

End If

ActiveSheet.Columns("B:Z").AutoFit

Worksheets("Energy Consumption").Activate

If Fuel $=1$ Then

MsgBox "Simulation is done" \& vbNewLine \& "MMBtu Consumption =" \& ActiveSheet.Range("I2") \& " MMBtu" \& vbNewLine \& " Electric Consumption (kWh)=" \& ActiveSheet.Range("H2") \& " kWh" \& vbNewLine \& " Electricity Costs $=\$$ " \& ActiveSheet.Range("H4") \& "" \& vbNewLine \& " NG Costs = \$ " \& ActiveSheet.Range("I4")

Else

MsgBox "Simulation is done" \& vbNewLine \& "Heater Consumption =" \& ActiveSheet.Range("I2") \& " kWh" \& vbNewLine \& " AC Energy Consumption (kWh)=" \& ActiveSheet.Range("H2") \& " kWh" \& vbNewLine \& " Heater Energy Costs $=\$$ " \& ActiveSheet.Range("H4") \& "" \& vbNewLine \& " AC Energy Costs $=\$ " \&$ ActiveSheet.Range("I4")

End If

Worksheets("Summary").Activate

ActiveSheet.Range("ALL1:ALL19000") = ""

End Sub 
